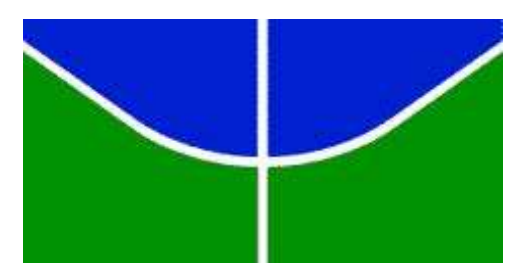

UNIVERSIDADE DE BRASÍLIA

INSTITUTO DE PSICOLOGIA

PROGRAMA DE PÓS-GRADUAÇÃO EM CIÊNCIAS DO COMPORTAMENTO

Avaliação de conceitos sobre dependência química entre estudantes de graduação na área de saúde da Universidade de Brasília

\author{
DISSERTAÇÃO DE MESTRADO
}

Karina Gomes Leal 
Universidade de Brasília

\title{
Avaliação de conceitos sobre dependência química entre estudantes de graduação na área de saúde da Universidade de Brasília
}

\author{
Karina Gomes Leal \\ Orientador: Prof. Dr. José Eduardo Pandossio \\ Dissertação apresentada ao Instituto de Psicologia \\ da Universidade de Brasília, como requisito parcial \\ para obtenção do grau de Mestre em Ciências do \\ Comportamento. Área de Cognição e \\ Neurociências do Comportamento.
}

Brasília 


\section{COMISSÃO EXAMINADORA}

Prof. Dr. André Amaral Bravin (Membro Efetivo)

Universidade Federal de Goiás (UFG), campus Jataí

Profa. Dra. Andrea Donatti Gallassi (Membro Efetivo) Faculdade de Ceilândia (FCE/UnB)

Prof. Dr. José Eduardo Pandossio (Presidente) Universidade de Brasília (UnB)

Prof. Dr. Francisco Dyonísio Cardoso Mendes (Suplente) Universidade de Brasília (UnB)

Brasília, 10 de fevereiro de 2017. 


\section{AGRADECIMENTOS}

À minha mãe, que me incentiva e apoia incondicionalmente. Grande companheira que nem mesmo nos momentos mais difíceis deixa de acreditar em mim.

Ao Caio, meu amor que caminha ao meu lado onde quer que eu esteja, custe o que custar, não importa a distância, nem o que aconteça, alegrando meus dias com sua ternura.

Ao Daniel e à Júlia, que aquecem meu coração com suas existências e que me dão força, alegria e orgulho constantes. Amor infinito pelas crianças mais deliciosas do planeta. Dedico a vocês esse trabalho.

Aos meus irmãos, Bruno, Júnior, Luíza, Marcelo e Taline, sempre presentes e cúmplices.

Aos meus queridos compadres Cristiano, Lindsay, Cadu, Nathália, Tiago, Amanda, Daniela, Gustavo e Stella, que compartilham comigo sonhos, momentos inesquecíveis e amor incondicional.

Aos meus amados Jurani, Sandra, Clodomir, Ariele, Tia Luluca, Guille, Camila, Ledox, Débora, Hellen, Rita e Vanja por me patrocinarem com seu carinho e me apoiarem na busca por meus objetivos.

Às amigas mais Simpáticas do mundo, Ana Clara, Anna Luíza, Bruna, Cristiane, Darla, Sara, Taize, Vanessa, Yamila, e famílias, que multiplicam alegrias e repartem o que há de melhor em si. Agradeço pelo grande companheirismo.

À minha afilhada Isabella, ao Davi e à Alice, pelo carinho e graciosidade que avivam meu coração.

Ao meu orientador, Eduardo, pela imensa contribuição na minha vida acadêmica e profissional, por me desafiar constantemente e pela generosidade durante a realização do nosso estudo. Grande exemplo de integridade e honradez.

Aos coordenadores de cursos e professores que assentiram me receber em suas turmas para a coleta dos dados, assim como aos estudantes que concordaram em responder ao questionário.

À banca examinadora, pela aceitação do convite e pela atenção dispensada na leitura desse trabalho. 


\section{RESUMO}

A dependência química é um transtorno psiquiátrico caracterizado por fissura pela droga, síndrome de abstinência, aumento da tolerância, relevância do consumo e empobrecimento do repertório de vida. Os estudantes de graduação da área de saúde deveriam receber capacitação sobre esse assunto, pois atuarão como parte da equipe multidisciplinar no tratamento da dependência. Este estudo avaliou os conceitos sobre dependência química abordados durante a graduação na área de saúde da Universidade de Brasília, com a participação de 944 alunos regularmente matriculados nos cursos de Ciências Farmacêuticas, Enfermagem, Farmácia, Fisioterapia, Fonoaudiologia, Gestão em Saúde Coletiva, Medicina, Nutrição, Odontologia, Psicologia, Saúde Coletiva e Terapia Ocupacional. Os resultados mostraram que os principais conceitos relativos à temática da dependência química e que o uso de drogas psicotrópicas pelos estudantes não apresentaram alterações depois que a(s) disciplina(s) da área de Farmacologia foram cursadas. Ademais, na comparação entre os grupos experimental e controle verificou-se que outras disciplinas, além daquelas da área de Farmacologia, promoveram o conhecimento sobre essa temática. A despeito de ambos os grupos terem cursado disciplina(s) que abordasse(m) esse tema, não foi verificada alteração quanto ao uso da maioria das drogas psicotrópicas pelos estudantes. Finalmente, esse estudo demonstra que faz-se necessária uma revisão de como a temática da dependência química é abordada nos cursos da área de saúde da Universidade de Brasília.

Palavras-chave: Dependência química, drogas psicotrópicas, universitários da área de saúde. 


\begin{abstract}
Chemical dependency is a psychiatric disorder characterized by drug fissure, withdrawal syndrome, increased tolerance, relevance of consumption and the life repertoire's impoverishment. University students in health area should receive training on this subject, once they will act as part of chemical dependency multidisciplinary treatment team. This study evaluated concepts of chemical dependency in health courses at Universidade of Brasília among 944 students regularly enrolled in Pharmaceutical Sciences, Nursing, Pharmacy, Physiotherapy, Speech Therapy, Public Health Management, Medicine, Nutrition, Dentistry, Psychology, Public Health and Occupational Therapy. Results showed that the main concepts regarding chemical dependency and psychotropic drugs used by students did not change after Pharmacology discipline attendance. In addition, comparison between experimental and control groups showed that other disciplines, besides those of Pharmacology, promoted knowledge about this subject. In spite of the fact that both groups have attended disciplines that discussed this theme, there was no change in most psychotropic drugs use by students. Finally, this study showed that it is necessary to review how chemical dependency subject is addressed in health area courses at Universidade de Brasília.
\end{abstract}

Keywords: Chemical dependency, psychotropic drugs, health area university students. 


\section{ÍNDICE}

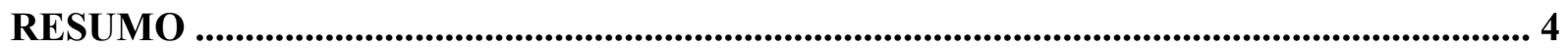

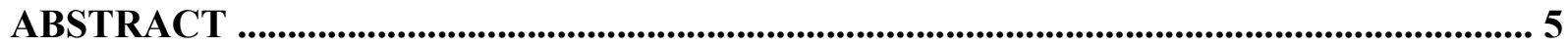

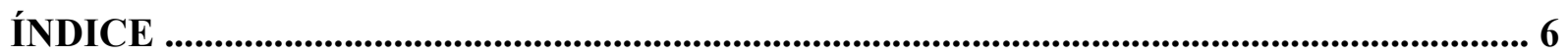

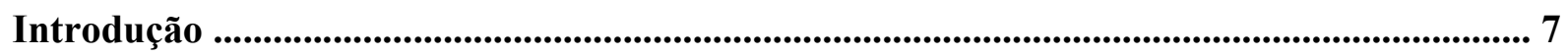

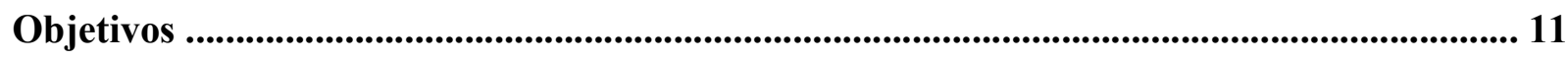

Materiais e método

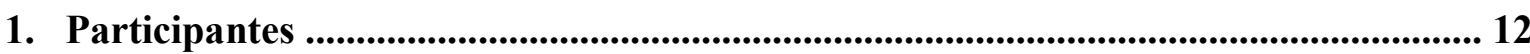

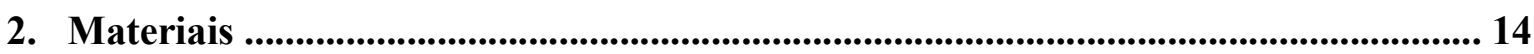

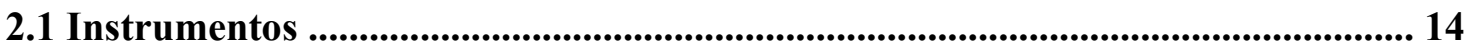

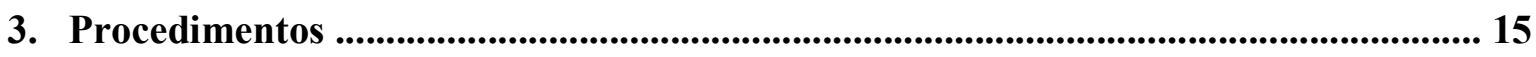

3.1 Aprovaçãa do projeto ........................................................................................................ 15

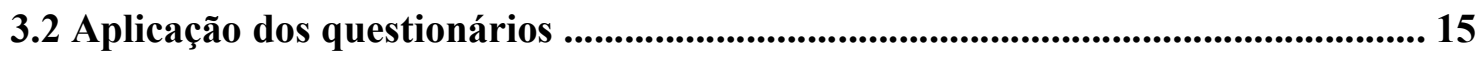

3.3 Avaliação dos questionários .............................................................................. 16

4. Análise de dados ............................................................................................................ 16

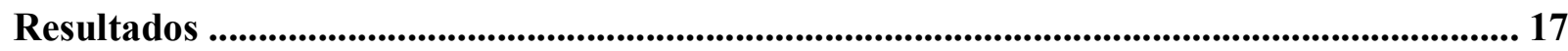

1. Avaliação das respostas dos questionários antes e após cursar a(s) disciplina(s) da

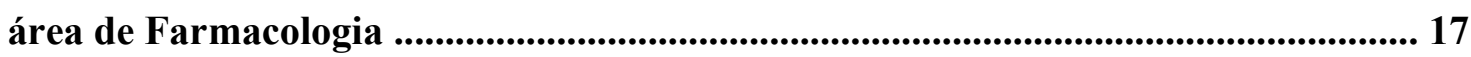

2. Avaliação da comparação entre os cursos do grupo experimental e do grupo controle

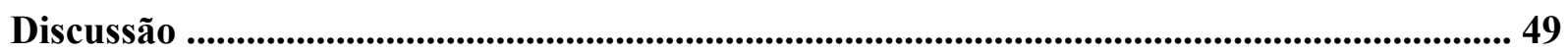

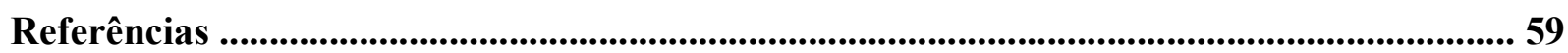

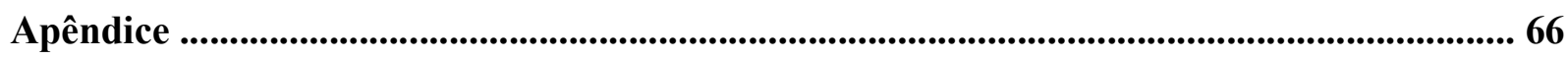




\section{Avaliação de conceitos sobre dependência química entre estudantes de graduação na área de saúde da Universidade de Brasília}

A dependência química é um transtorno psiquiátrico que leva a um padrão de consumo de drogas psicotrópicas que causa danos à saúde em médio e longo prazo, segundo a Organização Mundial da Saúde (OMS, 2006). De acordo com Diehl, Cordeiro e Laranjeira, (2010), o consumo que transgride normas sociais e compreende a utilização de substâncias ilícitas, o uso inadequado de drogas lícitas e/ou de medicamentos sem prescrição médica configura o abuso.

Os principais sinais e sintomas específicos da dependência química são apresentados na Classificação Internacional de Doenças (CID) - 10 (OMS, 2006) como fissura na ausência da droga, síndrome de abstinência, aumento da tolerância, relevância do consumo e estreitamento ou empobrecimento do repertório de vida. Desse modo, o padrão mal adaptado de uso de drogas psicotrópicas, aquelas que agem no sistema nervoso central e alteram comportamento, humor e cognição, possui grande propriedade reforçadora (OMS, 2006) e leva a perturbações clínicas associadas à dificuldade de interrompê-lo (Diehl et al., 2010).

Ainda, o consumo de drogas psicotrópicas oferece riscos secundários à população, como diminuição da expectativa de vida, acidentes automobilísticos, episódios de violência e comportamento sexual de risco, prejuízo nos desempenhos acadêmico e atlético, menor capacidade de se preparar para exames e/ou na participação em aulas, além de gerar absenteísmo, não conclusão, suspensão e retenção, atitude descomprometida com a universidade e professores, entre outros e por isso é considerado um problema de saúde (Martins et al., 2012; Brasil, 2010a). Vários levantamentos a respeito do padrão de consumo de drogas, como o Relatório Mundial sobre Drogas da United Nations Office on Drugs and Crime (UNODC, 2014) apontam que 243 milhões de pessoas, ou 5\% da população, entre 15 e 
64 anos de idade, usaram drogas ilícitas. Dependentes químicos somaram 27 milhões de pessoas, cerca de $0,6 \%$ da população adulta mundial ou 1 em cada 200 pessoas.

Conforme a Pesquisa Nacional de Saúde do Escolar, realizada pelo Instituto Brasileiro de Geografia e Estatística (IBGE, 2012), 40,1\% dos jovens brasileiros entre 18 e 24 anos frequentavam 2.252 instituições de ensino superior públicas e privadas, totalizando mais de 5,8 milhões de estudantes universitários. Ademais, o I Levantamento Nacional sobre o uso de Álcool, Tabaco e outras Drogas entre universitários das 27 Capitais Brasileiras registrou que $80 \%$ dos entrevistados afirmaram ter consumido bebida alcoólica, 46,7\% usaram produtos de tabaco e 49\% experimentaram alguma droga ilícita ao menos uma vez na vida (Brasil, 2010a). Isso reforça a assertiva de Carlini et al. (2007) de que nessa faixa etária apresentam-se as maiores frequências para o uso de drogas psicotrópicas e incidência de comportamentos de risco, tanto que conforme Kuo et al. (2002) existem outros levantamentos sobre o consumo de drogas entre estudantes universitários como a National College Health Risk Behavior Survey (NCHRBS), do Centers for Disease Control and Prevention (CDC), a College Alcohol Study, conduzida pela Harvard T.H. Chan School of Public Health e a National Survey on Drug Use and Health (NSDUH), realizada pelo Departament of Health and Human Services (HHS) do Substance Abuse and Mental Health Services Administration (SAMHSA).

Aliás, dados apresentados pelo European Monitoring Centre for Drugs and Drug Addiction (EMCDDA, 2014) mostram que o conhecimento sobre o uso de drogas psicotrópicas é importante, uma vez que na juventude se inicia o consumo e é nessa faixa etária que as atividades de prevenção têm melhores resultados. Logo, ao transmitir informações sobre dependência química para os jovens seria possível prevenir ou reduzir o uso de drogas psicotrópicas por meio de abordagens educacionais e informativas, em conformidade com o National Center on Addiction and Substance Abuse at Columbia University (CASA, 2007). 
Em 2001, o Conselho Nacional de Educação definiu, no parecer CNE/CES n. 1.300 (Brasil, 2004) que o objeto das diretrizes curriculares para os cursos de graduação em saúde seria construir um perfil acadêmico e profissional com competências e habilidades compatíveis com referências nacionais e internacionais e que formasse profissionais capazes de atuar com qualidade e eficiência no Sistema Único de Saúde (SUS). A despeito do estudo de Ramos et al. (2001), que aponta a importância do trabalho dos profissionais de saúde como parte da equipe interdisciplinar no tratamento da dependência química, esse parecer não emite juízo técnico sobre as características da capacitação que os estudantes da área deveriam receber, nem especifica os conteúdos a serem abordados durante a formação, ou como deveria ser o atendimento aos pacientes dependentes, por exemplo.

Ademais, Pillon, Siqueira e Silva (2011b) defendem que a situação atual relativamente ao treinamento sobre dependência química nos centros formadores na área de saúde no país é inadequada: seja por falta de exigência governamental acerca da capacitação nesse tópico, seja por discordância entre os estudiosos do tema quanto a um currículo mínimo que garantiria aos estudantes habilidades adequadas no manejo do paciente dependente. Em geral, os cursos na área de saúde abordam esse assunto durante determinada disciplina, mas a avaliação sobre essa formação não é realizada, nem existem parâmetros educacionais sobre a inclusão de técnicas, de modalidades de tratamentos e de outros aspectos relacionados à dependência química. Caberia aos cursos de graduação transmitir conhecimentos sobre identificação dos pacientes com problemas e preparar os estudantes para que façam encaminhamentos e/ou intervenções adequadas (Portugal, Souza, Buaiz \& Siqueira, 2008).

Por conseguinte, $28 \%$ das instituições participantes do I Levantamento Nacional sobre o uso de Álcool, Tabaco e outras Drogas entre universitários das 27 Capitais Brasileiras apresentaram alguma modalidade de programa ou projeto referente à prevenção e/ou orientação e/ou assistência de seus alunos no que se refere à temática em questão e um único 
programa especificou a necessidade de implementação de componentes curriculares sobre uso de drogas (Brasil, 2010a). A propósito, Carraro, Rassool e Luis (2005) apontam que as universidades são responsáveis por gerar e aplicar conhecimento, na forma de ensino ou pesquisa, e oferecer aos estudantes condições de adquirir competências para o exercício da profissão e prepará-los para atuar na área e para cuidar daquelas pessoas envolvidas neste contexto. Além disso, Boni, Pillon, Santos, Camata e Macieira (2004) afirmam que os profissionais da área de saúde têm contato com indivíduos com problemas de uso de substâncias psicotrópicas, de modo que os conceitos sobre dependência química e seu tratamento deveriam ser abordados durante a graduação.

Em vista disso, a educação em dependência química deveria ser entendida como um processo contínuo, também durante a atuação profissional, afinal a formação sobre dependência química poderia influenciar na prática inadequada e preconceituosa dos profissionais da área, declaram Pillon et al. (2011b). Destarte, no contexto específico da formação em saúde na Universidade de Brasília (UnB), os fluxos dos cursos Ciências Farmacêuticas, Enfermagem, Medicina, Nutrição, Odontologia e Psicologia, no Campus Darcy Ribeiro (Asa Norte) e Enfermagem e Farmácia, no Campus da Ceilândia, preveem que seus estudantes frequentem aulas que abordem o tema dependência química durante, no mínimo, um semestre (UnB, 2015b). De acordo com isso e tomando como base suas respectivas ementas, disciplinas como Farmacologia, Farmacologia Básica, Farmacologia 2, Toxicologia, Tópicos em Neurofarmacologia, Fundamentos em Farmacologia, Farmacologia Geral, Farmacologia Clínica, Toxicologia aplicada à Farmácia e Psicofarmacologia apresentariam conteúdos sobre uso e abuso de drogas, tratamentos para a dependência, redução de danos, entre outros.

Quanto ao consumo de drogas psicotrópicas por estudantes de graduação, Tockus e Gonçalves (2008) relatam que o ambiente universitário favorece o contato com drogas 
psicotrópicas e que seu uso tem relação com o contexto sociocultural dos graduandos, algo relevante e controverso para um grupo que tem acesso a mais informações técnicas que a população em geral. De modo semelhante, o estudo de Medeiros, Rediess, Hauck Filho, Martins e Mazoni, (2012) conclui que o consumo de álcool na vida ou nos últimos meses entre universitários brasileiros situa-se entre $80-90 \%$, enquanto $25-50 \%$ dos entrevistados consumiram nicotina e 30-50\% drogas ilícitas. Inclusive, os alunos da área de saúde seriam mais vulneráveis ao uso de drogas psicotrópicas em função do meio estressante em que estão inseridos, das exigências dos cursos de graduação e do acesso aos psicotrópicos, conforme Lucas et al. (2006).

Dessa forma, transmitir aos estudantes os conceitos da sintomatologia e do tratamento da dependência química seria uma forma de proporcionar cuidados efetivos e prevenir o agravamento dos problemas relacionados ao consumo de drogas psicotrópicas (Rassool, 2000). Ainda, Pillon et. al. (2010) reiteram que conceitos como os vários tipos de intervenção, interações entre drogas psicotrópicas, assim como o conhecimento sobre diferentes abordagens e modalidades de tratamento, como redução de danos, por exemplo, deveriam fazer parte da formação dos futuros profissionais da área.

\section{Objetivos}

O objetivo geral do presente estudo foi avaliar o conhecimento acerca dos conceitos sobre dependência química entre os estudantes da área de saúde da Universidade de Brasília.

Os objetivos específicos foram:

* Verificar, por meio de questionário, possíveis alterações em relação ao conhecimento dos estudantes da área de saúde da UnB envolvendo conceitos sobre dependência química, antes e depois de cursar a(s) disciplina(s) da área de Farmacologia, especificamente para os cursos que detêm, na sua grade curricular, essa(s) disciplina(s), nos 
campi Darcy Ribeiro (Ciências Farmacêuticas diurno e noturno, Medicina, Nutrição, Odontologia e Psicologia) e Ceilândia (Enfermagem e Farmácia).

* Avaliar, por meio de questionário, a relação entre a exposição aos conteúdos recebidos durante a graduação e o possível uso de drogas psicotrópicas por parte dos estudantes.

* Comparar os resultados dos questionários dos estudantes pertencentes aos cursos supracitados com aqueles dos estudantes que não possuem, em sua grade curricular, a(s) disciplina(s) da área de Farmacologia, pertencentes a outros cursos da área de saúde da UnB, nos campi Darcy Ribeiro (Gestão em Saúde Coletiva) e Faculdade da Ceilândia (Fisioterapia, Fonoaudiologia, Saúde Coletiva e Terapia Ocupacional), no sentido de verificar possíveis diferenças quanto ao conhecimento sobre dependência química.

\section{Materiais e Método}

\section{Participantes}

Para a verificação de possíveis alterações acerca do conhecimento envolvendo conceitos sobre dependência química, antes e depois de cursar a(s) disciplina(s) da área de Farmacologia, participaram desse estudo os estudantes regularmente matriculados nos cursos da área de saúde da Universidade de Brasília (UnB, 2015a) nos campi da Asa Norte (Darcy Ribeiro) e da Ceilândia. Esses foram separados em dois grupos, de acordo com o seguinte critério: (Antes) aqueles que ainda não haviam cursado a disciplina Farmacologia (ou equivalente na área) e (Depois) aqueles que já a haviam feito, como se vê no quadro abaixo:

Quadro 1: Distribuição participantes do grupo experimental por campi, cursos e semestres.

\begin{tabular}{lccc}
\hline Curso & Antes & Depois & N participantes \\
\hline & Campus Darcy Ribeiro & \\
\hline Ciências Farm. diurno & $2^{\mathrm{o}}$ semestre & $8^{\circ}$ semestre & 77 \\
Ciências Farm. noturno & $4^{\mathrm{o}}$ semestre & $9^{\mathrm{o}}$ semestre & 57 \\
Medicina & $2^{\mathrm{o}}$ semestre & $6^{\mathrm{o}}$ semestre & 49 \\
Nutrição & $4^{\mathrm{o}}$ semestre & $7^{\mathrm{o}}$ semestre & 69 \\
Odontologia & $2^{\mathrm{o}}$ semestre & $8^{\mathrm{o}}$ semestre & 46 \\
Psicologia & $4^{\mathrm{o}}$ semestre & $6^{\mathrm{o}}$ semestre & 95 \\
\hline
\end{tabular}




\begin{tabular}{llll}
\hline \multicolumn{5}{c}{ Campus Ceilândia } \\
\hline Enfermagem & $2^{\circ}$ semestre & $7^{\circ}$ semestre & 94 \\
Farmácia & $2^{\circ}$ semestre & $5^{\circ}$ semestre & 83 \\
\hline \multicolumn{5}{c}{ Total } & & $\mathbf{5 7 0}$ \\
\hline
\end{tabular}

No sentido de verificar se os estudantes da área de saúde que não têm em seu currículo a disciplina Farmacologia ou equivalente deteriam os conceitos sobre dependência química, também responderam os questionários os estudantes da Universidade de Brasília dos cursos listados no quadro a seguir:

Quadro 2: Distribuição participantes do grupo controle por campi, cursos e semestres.

\begin{tabular}{lcll}
\hline Curso & \multicolumn{3}{c}{ N participantes } \\
\hline Gestão em Saúde & $2^{\circ}$ semestre & $6^{\circ}$ semestre & 59 \\
Coletiva & \multicolumn{2}{c}{ Campus Ceilândia } \\
\hline Fisioterapia & $2^{\circ}$ semestre & $6^{\circ}$ semestre & 103 \\
Fonoaudiologia & $2^{\circ}$ semestre & $6^{\circ}$ semestre & 65 \\
Saúde Coletiva & $2^{\circ}$ semestre & $6^{\circ}$ semestre & 83 \\
Terapia Ocupacional & $2^{\circ}$ semestre & $6^{\circ}$ semestre & 64 \\
\hline \multicolumn{5}{c}{ Total } \\
\hline
\end{tabular}

Assim sendo, 944 questionários foram respondidos (n total). De acordo com o delineamento desse estudo, o número de participantes foi estimado em função do número de vagas de cada curso, ou seja, esperava-se obter, para cada curso, o dobro de participantes em relação ao número de vagas, o que nem sempre foi possível. Ainda, quando houve a comparação entre os cursos, nomeou-se de grupo experimental os cursos que apresentavam a(s) disciplina(s) de Farmacologia, enquanto que aqueles cursos que não a(s) apresentava(m) foram nomeados como grupo controle.

Os critérios de inclusão, neste estudo, foram: ser estudante de graduação da área de saúde da Universidade de Brasília, pertencer ao curso específico relativo à coleta determinada para aquele curso e ter 18 anos ou mais, enquanto os critérios de exclusão foram: não ser estudante de graduação da área de saúde da Universidade de Brasília, não pertencer ao curso específico relativo à coleta determinada para aquele curso e ser menor de 18 anos. 
Apesar dos contatos prévios com a coordenação do curso de Enfermagem do Campus Darcy Ribeiro, não houve autorização para que a coleta dos dados fosse realizada a tempo, somando-se o fato da ocupação de salas de aula no campus Darcy Ribeiro ${ }^{1}$ não havendo, portanto, condições de aplicar os questionários. Consequentemente, neste estudo, não constam os dados desse curso.

\section{Materiais}

\subsection{Instrumentos}

Foram utilizados, neste estudo, um Termo de Consentimento Livre e Esclarecido (TCLE) garantindo aos participantes o anonimato e a voluntariedade e, como instrumento, um questionário do tipo survey, composto por 25 perguntas. De acordo com Günther (2003), o questionário seria o instrumento principal para o levantamento de dados por amostragem, ou survey, assegurando melhor representatividade e permitindo a generalização para uma população mais ampla. Ainda nesse sentido, Fink e Kosecoff (1985), conforme citado por Günther (2003), definem survey como um “método para coletar informação de pessoas acerca de suas ideias, sentimentos, planos, crenças, bem como origem social, educacional e financeira" (p. 13). O conteúdo desse instrumento foi estruturado e adaptado para atender aos objetivos do presente estudo, como caracterização do curso universitário (período do curso), da opinião pessoal dos estudantes sobre o uso de drogas psicotrópicas (aprovar ou desaprovar o uso de determinada droga psicotrópica uma vez ou com frequência), das características da formação acadêmica recebida sobre o tema (aprendizado de conceitos, disciplinas cursadas), das drogas psicotrópicas utilizadas por eles (álcool, alucinógenos, esteroides anabolizantes, barbitúricos e benzodiazepínicos, cocaína, crack, inalantes, maconha, nicotina e Relevin -

\footnotetext{
${ }^{1}$ Notícias sobre a ocupação da Universidade de Brasília disponíveis em: http://www.correiobraziliense.com.br/app/noticia/euestudante/ensino_ensinosuperior/2016/10/31/ensino_ensinosuperior_interna,555533/alunos-da-unb-estao-nestemomento-em-assembleia-geral.shtml e http://www.correiobraziliense.com.br/app/noticia/cidades/2016/12/09/interna_cidadesdf,560618/desocupacaona-unb-sera-gradual-e-so-deve-terminar-em-13-de-dezembro.shtml.
} 
nome fictício de uma droga cuja marcação invalida o questionário, pois servia como parâmetro para assegurar a validade das respostas, seguindo as metodologias aplicadas em Brasil (2010a) e Brasil (2010b), e das opiniões sobre o tratamento da dependência química e acerca do dependente químico.

O questionário foi constituído por 24 questões fechadas, envolvendo alternativas de múltipla escolha em número variável, dependendo da temática de cada questão, e por uma última questão aberta, solicitando que o participante definisse, com apenas uma palavra, o dependente químico. Esse tipo de elaboração de questionário está de acordo com Günther (2003), ressaltando que, quando os tópicos mencionados pelos respondentes acerca de uma dada temática são conhecidos e, especialmente, quando existem muitos respondentes e/ou pouco tempo, deve-se optar por perguntas fechadas. Ainda, segundo Sommer e Sommer (1997), conforme citado por Günther (2003), o uso de perguntas fechadas “mostra frequentemente mais respeito à opinião das pessoas, deixando-as classificar suas respostas como positivas, negativas ou neutras, em vez do pesquisador fazer isto para eles” (p. 130). Da mesma maneira, perguntas abertas localizadas no final de um questionário teriam a função de capturar opiniões não cobertas pelos itens fechados (Günther, 2003).

\section{Procedimentos}

\subsection{Aprovação do Projeto}

O Projeto de Pesquisa n. 53583116.1.0000.5540 foi aprovado pelo Comitê de Ética em Pesquisa com Seres Humanos do Instituto de Ciências Humanas da Universidade de Brasília.

\subsection{Aplicação dos questionários}

Antes da aplicação dos questionários nos cursos especificados no delineamento, um teste piloto, com 12 estudantes do Programa de Pós-Graduação em Ciências do Comportamento, foi realizado a fim de avaliar possíveis problemas envolvendo o 
instrumento. Como o feedback desses alunos foi positivo, foram feitos pequenos ajustes e, em seguida, procedeu-se à aplicação do instrumento para a população-alvo.

Os dados foram coletados entre junho e dezembro de 2016. O questionário foi aplicado somente pela pesquisadora, de forma coletiva, em sala de aula, durante o tempo máximo de 15 minutos, estando a pesquisadora presente por todo o período da coleta, nas turmas que foram escolhidas seguindo os critérios de inclusão supracitados e cujos professores anuíram em recebê-la. Antes da distribuição do questionário, os estudantes leram o TCLE e foram informados sobre o objetivo do estudo e os procedimentos a que se submeteriam, podendo confirmar sua participação por meio de assinatura do TCLE. Aqueles que não concordaram com o TCLE e os menores de 18 anos foram convidados a se retirar da sala, permanecendo somente os estudantes que responderam os questionários, que foram depositados em envelope diferente daquele onde os TCLE assinados estavam guardados. A pesquisadora não respondeu dúvidas de conteúdo do instrumento durante a aplicação para evitar possíveis vieses de indução.

\subsection{Avaliação dos questionários}

Os dados de todos os participantes foram digitados eletronicamente para compor um banco de dados e as frequências (em porcentagem - \%) das respostas obtidas contabilizadas para cada curso.

\section{Análise dos dados}

Neste estudo, o teste $t$ não-pareado de Student $(\mathrm{p}<0,05)$ foi utilizado para identificar as diferenças significativas entre os estudantes que ainda não haviam cursado a(s) disciplina(s) da área de Farmacologia (grupo denominado Antes) e aqueles que já haviam cursado (grupo denominado Depois). Ao passo que as comparações intergrupos, entre os cursos da área de saúde da Universidade de Brasília avaliados neste estudo, foram realizadas pela Análise de Variância de uma via (ANOVA One-Way). O teste post-hoc usado para 
identificar as diferenças foi o teste de Student-Newman-Keuls $(\mathrm{p}<0,05)$. Para todas as análises, o software utilizado foi o SigmaStat@, versão 4.0 (2016).

\section{Resultados}

Os resultados estão apresentados em dois blocos: avaliação das respostas dos questionários antes e após cursar a(s) disciplina(s) da área de Farmacologia e avaliação da comparação entre os cursos do grupo experimental e do grupo controle. Apenas as respostas que obtiveram diferença significativa compõem a seção de resultados, sem necessariamente seguir a ordem numérica do questionário. As demais respostas encontram-se no Apêndice.

\section{Avaliação das respostas dos questionários antes e após cursar a(s) disciplina(s) da área de Farmacologia:}

Os resultados do teste $t$ não-pareado de Student foram obtidos a partir da análise da frequência das 570 respostas dos cursos Ciências Farmacêuticas (diurno e noturno), Enfermagem, Farmácia, Medicina, Nutrição, Odontologia e Psicologia, em comparação feita entre os dados dos estudantes Antes $(\mathrm{n}=281)$ de cursar a(s) disciplina(s) da área de Farmacologia e Depois $(\mathrm{n}=289)$.

\begin{tabular}{l}
$\begin{array}{l}\text { 6. Se você tivesse alguma dúvida a respeito de álcool, maconha ou } \\
\text { outras drogas, quem procuraria primeiro? (Marque apenas uma } \\
\text { alternativa) }\end{array}$ \\
\hline Professores \\
\hline Amigos ou colegas de faculdade \\
\hline Pais \\
\hline Serviço de Orientação da UnB \\
\hline Líder religioso (padre, pastor, rabino) \\
\hline Livros e/ou artigos científicos \\
\hline Internet
\end{tabular}

Na Questão 6, o teste $t$ não-pareado de Student revelou que houve diferença significativa entre as respostas obtidas em Livros elou artigos cientificos Antes $(\bar{X}=6.13$; $\mathrm{EPM}=0.74)$ e Depois $(\bar{X}=9.50 ; \mathrm{EPM}=1.23), t=-2.34$ e $\mathrm{p}=0.03$. Todavia, não houve diferença significativa nas respostas obtidas em Professores no Antes $(\bar{X}=2 ; \mathrm{EPM}=0.56) \mathrm{e}$ 
no Depois $(\bar{X}=3.88 ; \mathrm{EPM}=1.17), t=-1.44$ e $\mathrm{p}=0.17$, Amigos ou colegas de faculdade no

Antes $(\bar{X}=3.38 ; \mathrm{EPM}=0.92)$ e no Depois $(\bar{X}=4.75 ; \mathrm{EPM}=0.79), t=-1.13$ e $\mathrm{p}=0.27$,

Pais no Antes $(\bar{X}=2.38 ; \mathrm{EPM}=0.73)$ e no Depois $(\bar{X}=2.63 ; \mathrm{EPM}=1.52), t=0.14$ e $\mathrm{p}=$

0.88 , Serviço de Orientação da UnB no Antes $(\bar{X}=0 ; \mathrm{EPM}=0)$ e no Depois $(\bar{X}=0 ; \mathrm{EPM}=$ $0), t=0$ e $\mathrm{p}=1$, Líder religioso no Antes $(\bar{X}=0 ; \mathrm{EPM}=0)$ e no Depois $(\bar{X}=0.38 ; \mathrm{EPM}=$ $0.26), t=-1.43$ e $\mathrm{p}=0.17$ e Internet no Antes $(\bar{X}=21.9 ; \mathrm{EPM}=3.61)$ e no Depois $(\bar{X}=$ 15.6; $\mathrm{EPM}=1.91), t=1.53$ e $\mathrm{p}=0.14($ Figura 1$)$.

\section{Quem/o que procuraria primeiro em caso de dúvida sobre uso de drogas}

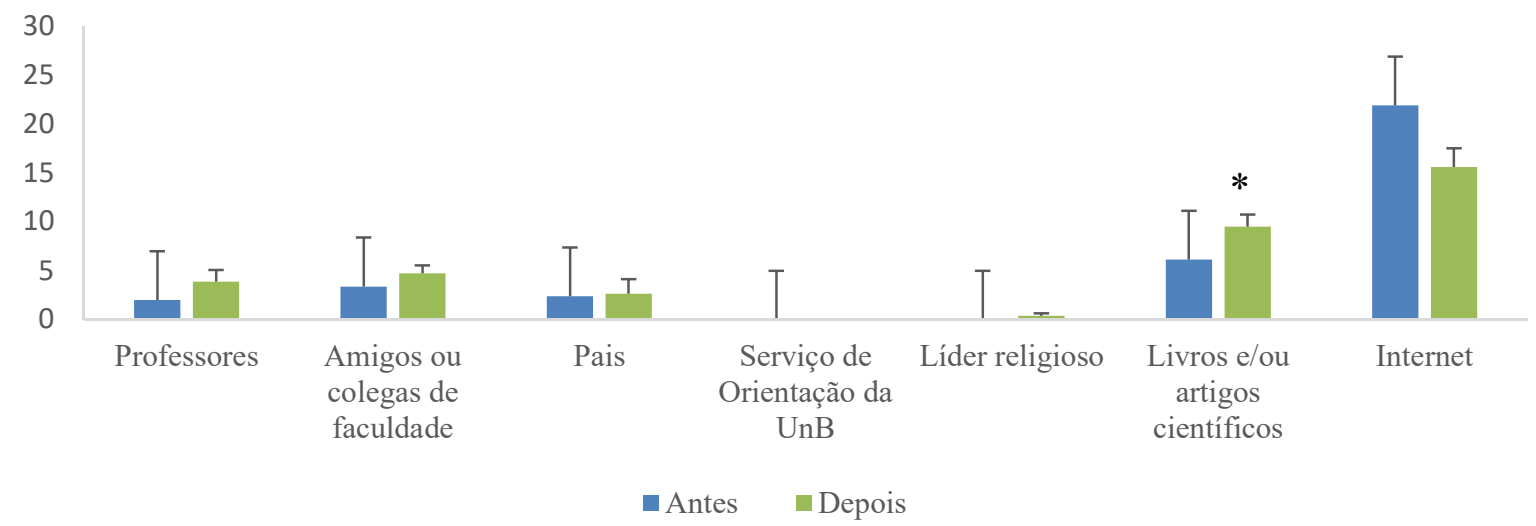

Figura 1: Quem/o que o estudante procuraria primeiro em caso de dúvida sobre uso de drogas antes e depois de cursar a(s) disciplina(s) da área de Farmacologia. As colunas representam as médias e as barras o EPM. * Indica diferença significativa na alternativa Livros e/ou artigos científicos Antes e Depois. $\mathrm{p}<0,05$, de acordo com o teste $t$ não-pareado de Student. Antes (n $=289)$ e Depois $(\mathrm{n}=281)$.

\begin{tabular}{l} 
7. O que você pensa a respeito de uma pessoa usar uma única vez \\
as seguintes substâncias? (Marque apenas aprovo, desaprovo ou \\
não sei para cada uma das substâncias) \\
\hline Álcool \\
\hline Benzodiazepínicos e barbitúricos \\
\hline Cigarro de nicotina \\
\hline Cigarro de maconha \\
\hline Cocaína \\
\hline Crack \\
\hline Drogas sintéticas \\
\hline Esteróides anabolizantes \\
\hline Inalantes
\end{tabular}


Na Questão 7, o teste $t$ não-pareado de Student revelou que houve diferença significativa apenas entre as respostas obtidas em aprovo Esteroides anabolizantes Antes ( $\bar{X}=2.25 ; \mathrm{EPM}=0.52)$ e Depois $(\bar{X}=10.38 ; \mathrm{EPM}=3.69), t=-2.18$ e $\mathrm{p}=0.04$. As demais alternativas não apresentaram diferença significativa: Álcool Antes $(\bar{X}=28.8 ; \mathrm{EPM}=3.83) \mathrm{e}$ Depois $(\bar{X}=28.3 ; \mathrm{EPM}=1.94), t=0.11$ e $\mathrm{p}=0.90$, Benzodiazepínicos e barbitúricos Antes $(\bar{X}=5.88 ; \mathrm{EPM}=1.90)$ e Depois $(\bar{X}=10.25 ; \mathrm{EPM}=2.42), t=-1.42$ e $\mathrm{p}=0.17$, Cigarro de nicotina Antes $(\bar{X}=9.38 ; \mathrm{EPM}=2.56)$ e Depois $(\bar{X}=11.25 ; \mathrm{EPM}=1.86), t=-0.59$ e $\mathrm{p}=$ 0.56, Cigarro de maconha Antes $(\bar{X}=12.3 ; \mathrm{EPM}=3.61)$ e Depois $(\bar{X}=12.4 ; \mathrm{EPM}=1.57)$, $t=-0.03$ e $\mathrm{p}=0.97$, Cocaina Antes $(\bar{X}=1.38 ; \mathrm{EPM}=0.49)$ e Depois $(\bar{X}=9.25 ; \mathrm{EPM}=$ 4.35), $t=-1.80$ e $\mathrm{p}=0.09$, Crack Antes $(\bar{X}=0.25 ; \mathrm{EPM}=0.16)$ e Depois $(\bar{X}=8.12 ; \mathrm{EPM}=$ 4.48), $t=-1.75$ e $\mathrm{p}=0.10$, Drogas sintéticas Antes $(\bar{X}=3.50 ; \mathrm{EPM}=1.18)$ e Depois $(\bar{X}=6$; $\mathrm{EPM}=1.43), t=-1.35$ e $\mathrm{p}=0.19$ e Inalantes Antes $(\bar{X}=2.25 ; \mathrm{EPM}=0.75)$ e Depois $(\bar{X}=$ $3.38 ; \mathrm{EPM}=0.90), t=-0.95$ e $\mathrm{p}=0.35($ Figura 2$)$.

\section{Aprovação do uso de substâncias uma única vez}

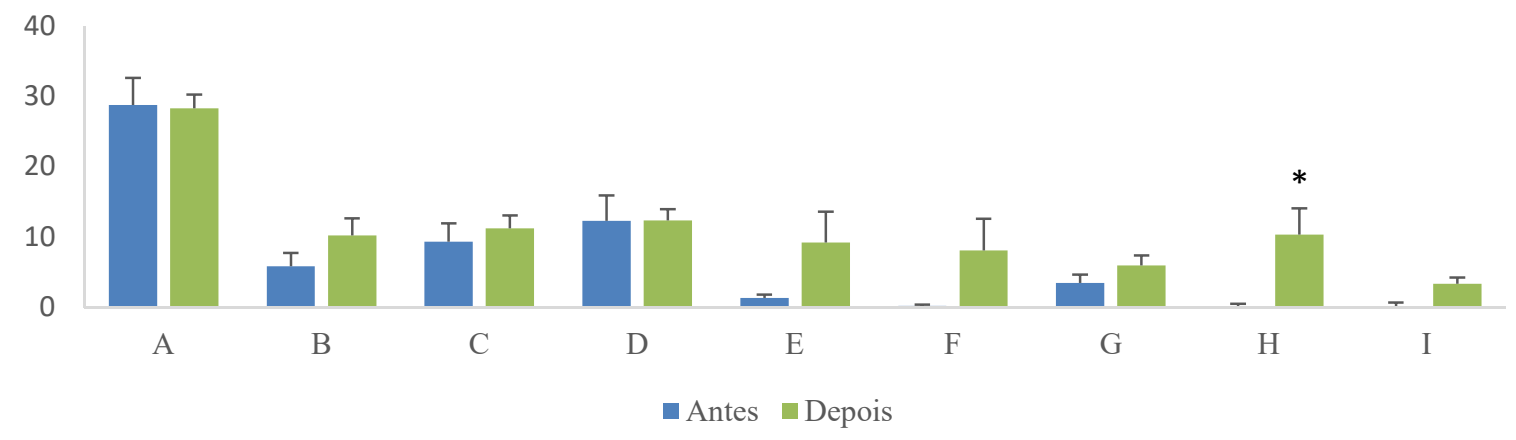

Figura 2: Aprovação do uso de substâncias uma única vez antes e depois de cursar a(s) disciplina(s) da área de Farmacologia. As colunas representam as médias das alternativas $\mathrm{A}=$ Álcool, $\mathrm{B}=$ Benzodiazepínicos e barbitúricos, $\mathrm{C}=$ Cigarro de nicotina, $\mathrm{D}=$ Cigarro de maconha, $\mathrm{E}=$ Cocaína, $\mathrm{F}=\mathrm{Crack}, \mathrm{G}=$ Drogas sintéticas, $\mathrm{H}=$ Esteroides anabolizantes, $\mathrm{I}=$ Inalantes e as barras o EPM. * Indica diferença significativa na alternativa aprovo experimentar Esteroides anabolizantes Antes e Depois. $\mathrm{p}<0,05$, de acordo com o teste $t$ nãopareado de Student. Antes $(\mathrm{n}=289)$ e Depois $(\mathrm{n}=281)$. 


\begin{tabular}{l}
\hline Álcool \\
\hline Benzodiazepínicos e barbitúricos \\
\hline Cigarro de nicotina \\
\hline Cigarro de maconha \\
\hline Cocaína \\
\hline Crack \\
\hline Drogas sintéticas \\
\hline Esteróides anabolizantes \\
\hline Inalantes
\end{tabular}

Na Questão 8, acerca da aprovação do uso frequente das substâncias acima relacionadas, o teste $t$ não-pareado de Student mostrou que houve diferença significativa apenas entre as respostas da alternativa Benzodiazepínicos e barbitúricos Antes $(\bar{X}=0.87$; $\mathrm{EPM}=0.39)$ e Depois $(\bar{X}=2.25 ; \mathrm{EPM}=0.45), t=-2.28$ e $\mathrm{p}=0.03$. As outras respostas obtidas não apresentaram diferença significativa: Álcool Antes $(\bar{X}=9.25 ; \mathrm{EPM}=2.16) \mathrm{e}$ Depois $(\bar{X}=10 ; \mathrm{EPM}=0.98), t=-0.31$ e $\mathrm{p}=0.75$, Cigarro de nicotina Antes $(\bar{X}=2.63$; $\mathrm{EPM}=1.01)$ e Depois $(\bar{X}=2.25 ; \mathrm{EPM}=0.86), t=0.28$ e $\mathrm{p}=0.78$, Cigarro de maconha Antes $(\bar{X}=4.75 ; \mathrm{EPM}=1.32)$ e Depois $(\bar{X}=4.13 ; \mathrm{EPM}=1.26), t=0.34$ e $\mathrm{p}=0.73$, Cocaina Antes $(\bar{X}=0.25 ; \mathrm{EPM}=0.16)$ e Depois $(\bar{X}=0.37 ; \mathrm{EPM}=0.26), t=0.40$ e $\mathrm{p}=$ 0.69, Crack Antes $(\bar{X}=0 ; \mathrm{EPM}=0)$ e Depois $(\bar{X}=0.25 ; \mathrm{EPM}=0.16), t=-1.53$ e p $=0.14$, Drogas sintéticas Antes $(\bar{X}=1 ; \mathrm{EPM}=0.26)$ e Depois $(\bar{X}=0.87 ; \mathrm{EPM}=0.39), t=0.26$ e p $=0.79$, Esteroides anabolizantes Antes $(\bar{X}=0.87 ; \mathrm{EPM}=0.22)$ e Depois $(\bar{X}=0.37 ; \mathrm{EPM}=$ $0.26), t=1.44$ e $\mathrm{p}=0.17$ e Inalantes Antes $(\bar{X}=0.62 ; \mathrm{EPM}=0.18)$ e Depois $(\bar{X}=0.50$; $\mathrm{EPM}=0.26), t=0.38$ e $\mathrm{p}=0.70($ Figura 3$)$.

\section{Aprovação do uso frequente de substâncias}

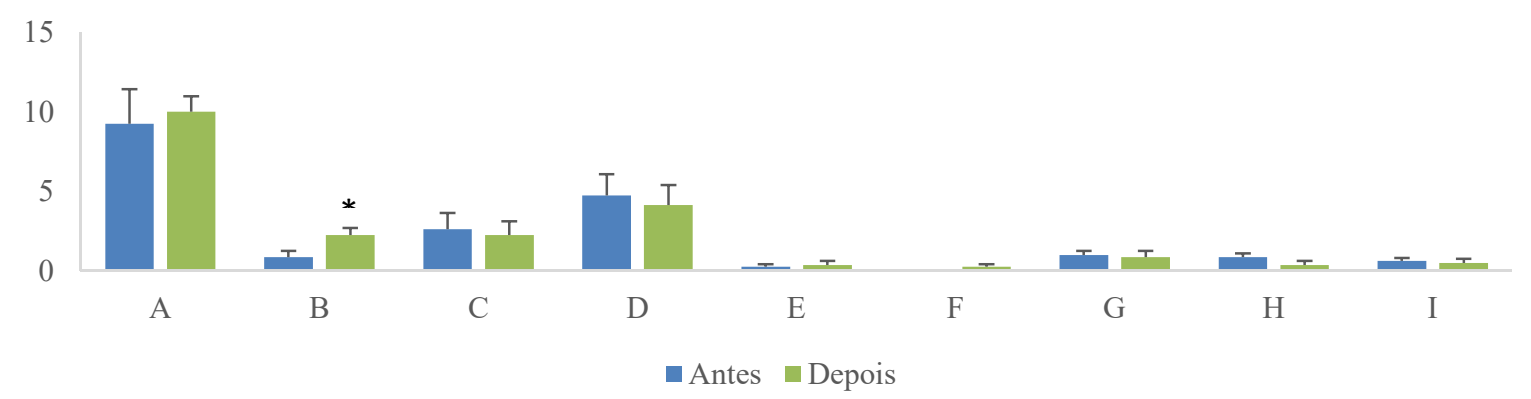

Figura 3: Aprovação do uso frequente de substâncias antes e depois de cursar a(s) disciplina(s) da área de Farmacologia. As colunas representam as médias das alternativas A = 
Álcool, $\mathrm{B}=$ Benzodiazepínicos e barbitúricos, $\mathrm{C}=$ Cigarro de nicotina, $\mathrm{D}=$ Cigarro de maconha, $\mathrm{E}=$ Cocaína, $\mathrm{F}=$ Crack, $\mathrm{G}=$ Drogas sintéticas, $\mathrm{H}=$ Esteroides anabolizantes, $\mathrm{I}=$ Inalantes e as barras o EPM. * Indica diferença significativa na alternativa aprovo usar frequentemente Benzodiazepínicos e barbitúricos Antes e Depois. $\mathrm{p}<0,05$, de acordo com o teste $t$ não-pareado de Student. Antes $(\mathrm{n}=289)$ e Depois $(\mathrm{n}=281)$.

Com relação à opção não sei sobre o uso frequente das substâncias listadas acima, o teste $t$ não-pareado de Student revelou que houve diferença somente para a alternativa Cocaina Antes $(\bar{X}=0.50 ; \mathrm{EPM}=1.37)$ e Depois $(\bar{X}=1.37$; EPM $=0.32), t=-2.08$ e $\mathrm{p}=$ 0.05. As demais alternativas não tiveram diferença estatisticamente significativa: Álcool Antes $(\bar{X}=3.88 ; \mathrm{EPM}=0.47)$ e Depois $(\bar{X}=4.63 ; \mathrm{EPM}=1.28), t=-0.54$ e $\mathrm{p}=0.59$, Benzodiazepínicos e barbitúricos Antes $(\bar{X}=13.75 ; \mathrm{EPM}=2.96)$ e Depois $(\bar{X}=9.13$; EPM $=1.69), t=1.36$ e p $=0.19$, Cigarro de nicotina Antes $(\bar{X}=2 ; \mathrm{EPM}=0.53)$ e Depois $(\bar{X}=$ 1.50; $\mathrm{EPM}=0.35), t=0.66 \mathrm{e} \mathrm{p}=0.51$, Cigarro de maconha Antes $(\bar{X}=3.13 ; \mathrm{EPM}=0.85) \mathrm{e}$ Depois $(\bar{X}=2.75 ; \mathrm{EPM}=0.70), t=1.33$ e $\mathrm{p}=0.73$, Crack Antes $(\bar{X}=0.62 ; \mathrm{EPM}=0.18)$ e Depois $(\bar{X}=1.12 ; \mathrm{EPM}=0.35), t=-1.26$ e $\mathrm{p}=0.22$, Drogas sintéticas Antes $(\bar{X}=4.38$; $\mathrm{EPM}=1.26)$ e Depois $(\bar{X}=2.63 ; \mathrm{EPM}=0.80), t=1.17$ e $\mathrm{p}=0.26$, Esteroides anabolizantes Antes $(\bar{X}=1.50 ; \mathrm{EPM}=0.37)$ e Depois $(\bar{X}=2.75 ; \mathrm{EPM}=0.64), t=-1.67$ e $\mathrm{p}=0.11$ e Inalantes Antes $(\bar{X}=3.25 ; \mathrm{EPM}=1.13)$ e Depois $(\bar{X}=2.88 ; \mathrm{EPM}=0.44), t=0.30$ e $\mathrm{p}=$ 0.76 (Figura 4).

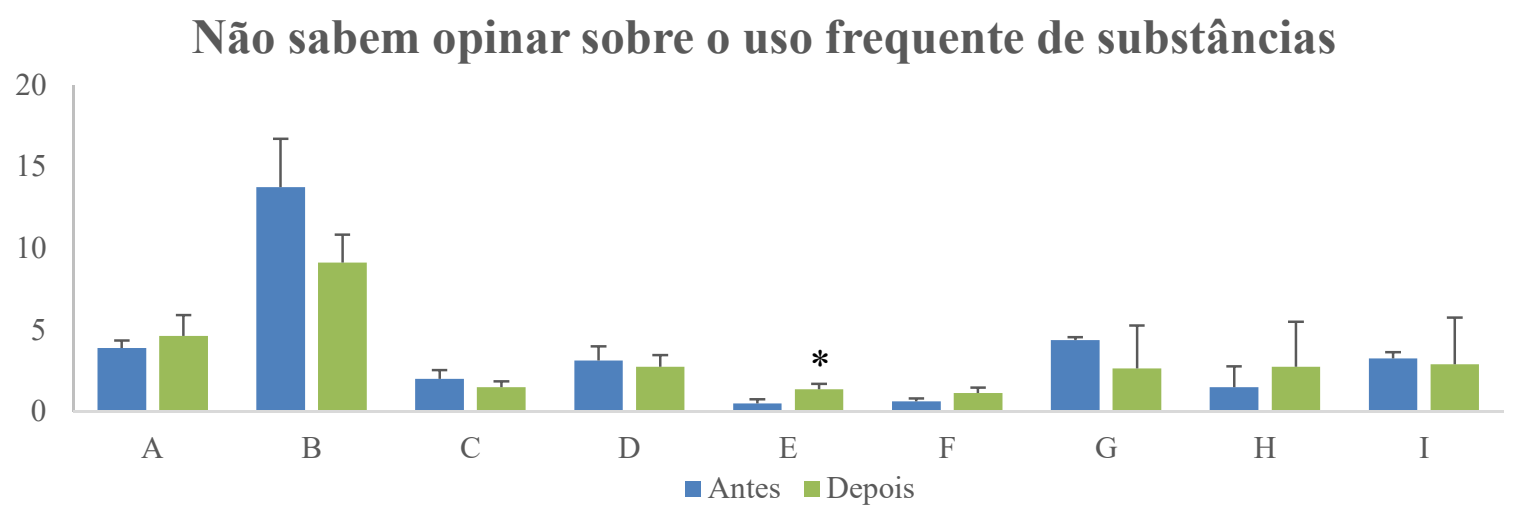

Figura 4: Alternativa não sei sobre o uso frequente de substâncias antes e depois de cursar $\mathrm{a}(\mathrm{s})$ disciplina(s) da área de Farmacologia. As colunas representam as médias das alternativas $\mathrm{A}=$ Álcool, $\mathrm{B}=$ Benzodiazepínicos e barbitúricos, $\mathrm{C}=$ Cigarro de nicotina, $\mathrm{D}=$ Cigarro de maconha, $\mathrm{E}=$ Cocaína, $\mathrm{F}=$ Crack, $\mathrm{G}=$ Drogas sintéticas, $\mathrm{H}=$ Esteroides anabolizantes, $\mathrm{I}=$ Inalantes e as barras o EPM. * Indica diferença significativa na opção não sei a respeito de 
usar frequentemente Cocaína Antes e Depois. $\mathrm{p}<0,05$, de acordo com o teste $t$ não-pareado de Student. Antes $(n=289)$ e Depois $(n=281)$.

\begin{tabular}{l}
\hline $\begin{array}{l}\text { 10. Como você vê a dependência química? (Assinale todas as } \\
\text { alternativas que se aplicam) }\end{array}$ \\
\hline Indica fraqueza moral \\
\hline Mostra a falta de vontade de mudar \\
\hline Pode ser combatida pela fé \\
\hline Tem cura \\
\hline Tem tratamento \\
\hline Nenhuma das anteriores \\
\hline
\end{tabular}

Na Questão 10, o teste $t$ não-pareado de Student revelou que houve diferença significativa apenas entre as respostas obtidas em Mostra falta de vontade de mudar Antes ( $\bar{X}=3.63 ; \mathrm{EPM}=0.73)$ e Depois $(\bar{X}=1.75 ; \mathrm{EPM}=0.31), t=2.36$ e $\mathrm{p}=0.03$. Portanto, as respostas obtidas nas demais alternativas não apresentaram diferença significativa: Indica fraqueza moral Antes $(\bar{X}=4.88 ; \mathrm{EPM}=1.10)$ e Depois $(\bar{X}=3.13 ; \mathrm{EPM}=0.91), t=1.22$ e $\mathrm{p}$ $=0.24$, Pode ser combatida pela fé Antes $(\bar{X}=7.63 ; \mathrm{EPM}=0.68)$ e Depois $(\bar{X}=6.50 ; \mathrm{EPM}$ $=1.62), t=0.63$ e p $=0.53$, Tem cura Antes $(\bar{X}=13.3 ; \mathrm{EPM}=1.92)$ e Depois $(\bar{X}=12.3$; $\mathrm{EPM}=1.59), t=0.40$ e $\mathrm{p}=0.69$, Tem tratamento Antes $(\bar{X}=33.1 ; \mathrm{EPM}=4.31)$ e Depois ( $\bar{X}=32 ; \mathrm{EPM}=3.18), t=0.21$ e $\mathrm{p}=0.83$ e Nenhuma das anteriores Antes $(\bar{X}=1.63 ; \mathrm{EPM}$ $=0.26)$ e Depois $(\bar{X}=1.88 ; \mathrm{EPM}=0.63), t=-0.36$ e $\mathrm{p}=0.72($ Figura 5$)$.

\section{Justificativas sobre a dependência química}

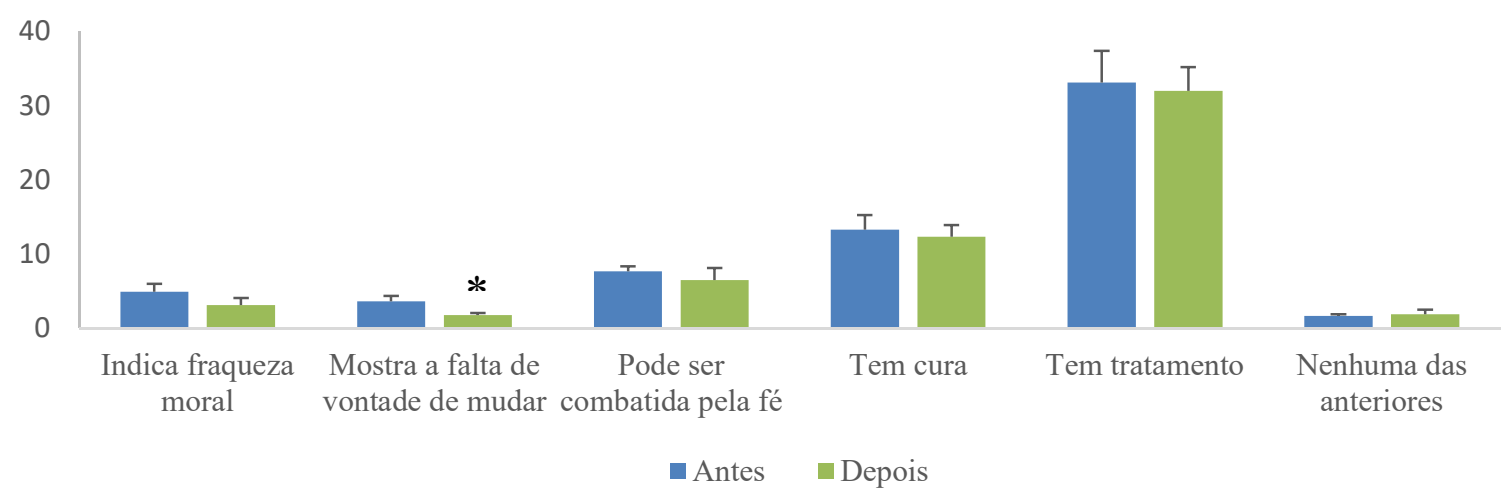

Figura 5: Justificativas dos estudantes sobre a dependência química. As colunas representam as médias e as barras o EPM. * Indica diferença significativa na alternativa Mostra falta de vontade de mudar Antes e Depois. $\mathrm{p}<0,05$, de acordo com o teste $t$ não-pareado de Student. Antes $(\mathrm{n}=289)$ e Depois $(\mathrm{n}=281)$. 


\begin{tabular}{l}
$\begin{array}{l}\text { 19. Você usou alguma das substâncias da questão } 17 \text { no último } \\
\text { ano? }\end{array}$ \\
\hline Sim \\
\hline Não \\
\hline Não se aplica
\end{tabular}

Na Questão 19, o teste $t$ não-pareado de Student mostrou que houve diferença significativa apenas entre as respostas obtidas na alternativa Não se aplica Antes $(\bar{X}=4$; $\mathrm{EPM}=0.80)$ e Depois $(\bar{X}=2 ; \mathrm{EPM}=0.46), t=2.16$ e $\mathrm{p}=0.04$. Enquanto as respostas das alternativas $\operatorname{Sim}$ Antes $(\bar{X}=27.9 ; \mathrm{EPM}=3.74)$ e Depois $(\bar{X}=28 ; \mathrm{EPM}=1.69), t=-0.03 \mathrm{e}$ $\mathrm{p}=0.97$ e Não Antes $(\bar{X}=3.88 ; \mathrm{EPM}=0.99)$ e Depois $(\bar{X}=5.25 ; \mathrm{EPM}=1.13), t=-0.91$ e $p=0.37$ não apresentaram diferença significativa (Figura 6).

\section{Uso de droga psicotrópica no último ano}

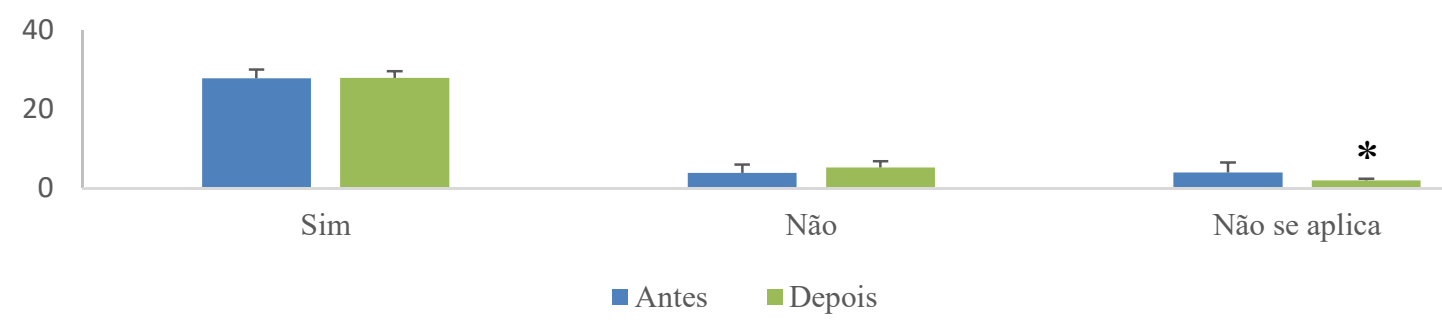

Figura 6: Uso de drogas psicotrópicas no último ano em comparação entre antes e depois de cursar a(s) disciplina(s) da área de Farmacologia. As colunas representam as médias e as barras o EPM. * Indica diferença significativa na alternativa Não se aplica Antes e Depois. p $<0,05$, de acordo com o teste $t$ não-pareado de Student. Antes $(\mathrm{n}=289)$ e Depois $(\mathrm{n}=281)$.

\begin{tabular}{l}
\hline $\begin{array}{l}\text { 20. Que motivo o levou a fazer uso de alguma das substâncias citadas } \\
\text { anteriormente pela primeira vez? (Assinale todas as alternativas que se } \\
\text { aplicam) }\end{array}$ \\
\hline Alívio de cansaço \\
\hline Alívio de dor \\
\hline Aumentar desempenho de estudo \\
\hline Curiosidade \\
\hline Diversão/prazer \\
\hline Manter-se acordado(a) \\
\hline Melhorar desempenho sexual \\
\hline Perder inibição \\
\hline Porque amigos/namorado(a) usam \\
\hline Prática religiosa \\
\hline Relaxar \\
\hline Outro
\end{tabular}

Na Questão 20, o teste $t$ não-pareado de Student revelou que houve diferença significativa apenas na alternativa Melhorar desempenho sexual Antes $(\bar{X}=0.12 ; \mathrm{EPM}=$ 
$0.12)$ e Depois $(\bar{X}=0.62 ; \mathrm{EPM}=0.18), t=-2.26$ e $\mathrm{p}=0.04$. As demais respostas não apresentaram diferença significativa: Alívio de cansaço Antes $(\bar{X}=2.88 ; \mathrm{EPM}=0.78) \mathrm{e}$ Depois $(\bar{X}=4.50 ; \mathrm{EPM}=0.77), t=-1.47 \mathrm{e} \mathrm{p}=0.16$, Alivio de dor Antes $(\bar{X}=1.88 ; \mathrm{EPM}=$ $0.54)$ e Depois $(\bar{X}=3.50 ; \mathrm{EPM}=0.75), t=-1.74$ e $\mathrm{p}=0.10$, Aumentar desempenho de estudo Antes $(\bar{X}=0.87 ; \mathrm{EPM}=0.29)$ e Depois $(\bar{X}=1.12 ; \mathrm{EPM}=0.22), t=-0.67$ e $\mathrm{p}=$ 0.51, Curiosidade Antes $(\bar{X}=17.3 ; \mathrm{EPM}=3.14)$ e Depois $(\bar{X}=16.9 ; \mathrm{EPM}=1.80), t=0.10$ e $\mathrm{p}=0.91$, Diversão/prazer Antes $(\bar{X}=25.4 ; \mathrm{EPM}=3.31)$ e Depois $(\bar{X}=24.6 ; \mathrm{EPM}=$ $1.22), t=0.21$ e p $=0.83$, Manter-se acordado $(a)$ Antes $(\bar{X}=1.37 ; \mathrm{EPM}=0.32)$ e Depois ( $\bar{X}=0.87 ; \mathrm{EPM}=0.35), t=1.05$ e $\mathrm{p}=0.31$, Perder inibição Antes $(\bar{X}=5.75 ; \mathrm{EPM}=1.71) \mathrm{e}$ Depois $(\bar{X}=6.38 ; \mathrm{EPM}=1.21), t=-0.29$ e $\mathrm{p}=0.76$ Porque amigos $/$ namorado(a) usam Antes $(\bar{X}=5.63 ; \mathrm{EPM}=1.12)$ e Depois $(\bar{X}=5.63 ; \mathrm{EPM}=1.16), t=0$ e $\mathrm{p}=1$, Prática religiosa Antes $(\bar{X}=0.37 ; \mathrm{EPM}=0.26)$ e Depois $(\bar{X}=0.75 ; \mathrm{EPM}=0.41), t=-0.76$ e $\mathrm{p}=$ 0.45, Relaxar Antes $(\bar{X}=11.4 ; \mathrm{EPM}=1.28)$ e Depois $(\bar{X}=11.1 ; \mathrm{EPM}=1.60), t=0.12$ e p $=0.90$ e Outro Antes $(\bar{X}=3.25 ; \mathrm{EPM}=0.81)$ e Depois $(\bar{X}=2.38 ; \mathrm{EPM}=0.37), t=0.97$ e p $=0.34$ (Figura 7$)$.

\section{Motivos para o uso de drogas psicotrópicas entre estudantes}

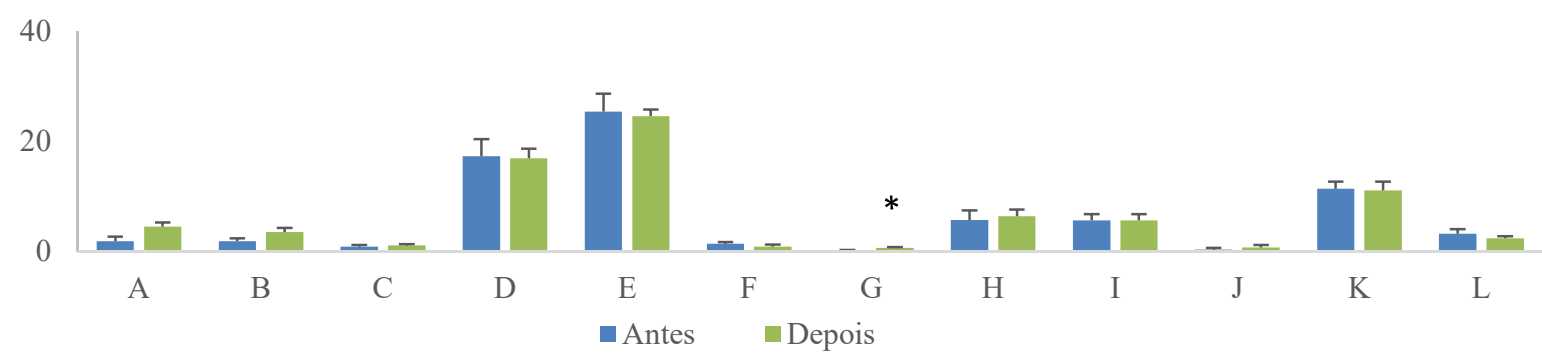

Figura 7: Motivos para o uso de drogas psicotrópicas entre os estudantes. As colunas representam as médias das alternativas: A - Alívio de cansaço, B - Alívio de dor, C Aumentar desempenho de estudo, D - Curiosidade, E - Diversão/prazer, F - Manter-se acordado(a), G - Melhorar desempenho sexual, H - Perder inibição, I - Porque amigos/namorado(a) usam, J - Prática religiosa, K - Relaxar, L - Outro e as barras o EPM. * Indica diferença significativa na alternativa Melhorar desempenho sexual Antes e Depois. $\mathrm{p}<$ 0,05 de acordo com o teste $t$ não-pareado de Student. Antes $(\mathrm{n}=289)$ e Depois $(\mathrm{n}=281)$. 


\begin{tabular}{l}
\hline Não tem eficácia \\
\hline Pode envolver tratamento medicamentoso \\
\hline Pode envolver uso de droga que cause dependência \\
\hline Tem como objetivo a abstinência \\
\hline Não sei \\
\hline
\end{tabular}

Na Questão 24, o teste $t$ não-pareado de Student revelou que houve diferença significativa apenas entre as respostas obtidas em Não sei Antes $(\bar{X}=3.63 ; \mathrm{EPM}=0.73) \mathrm{e}$ Depois $(\bar{X}=1.75 ; \mathrm{EPM}=0.31), t=2.36 \mathrm{e} \mathrm{p}=0.03$. As respostas das demais alternativas não apresentaram diferença significativa: Não tem eficácia Antes $(\bar{X}=0.12 ; \mathrm{EPM}=0.12) \mathrm{e}$ Depois $(\bar{X}=0.12 ; \mathrm{EPM}=0.12), t=0$ e $\mathrm{p}=1$, Pode envolver tratamento medicamentoso Antes $(\bar{X}=1.88 ; \mathrm{EPM}=0.81)$ e Depois $(\bar{X}=5.38 ; \mathrm{EPM}=.022), t=-1.60$ e $\mathrm{p}=0.13$, Pode envolver uso de droga que cause dependência Antes $(\bar{X}=2.25 ; \mathrm{EPM}=1.33)$ e Depois $(\bar{X}=$ $7.75 ; \mathrm{EPM}=3.54), t=-1.45$ e $\mathrm{p}=0.16$ e Tem como objetivo a abstinência Antes $(\bar{X}=0.12$; $\mathrm{EPM}=0.12)$ e Depois $(\bar{X}=0.50 ; \mathrm{EPM}=0.18), t=-1.66$ e $\mathrm{p}=0.12$ (Figura 8$).$

\section{Características sobre a redução de danos}

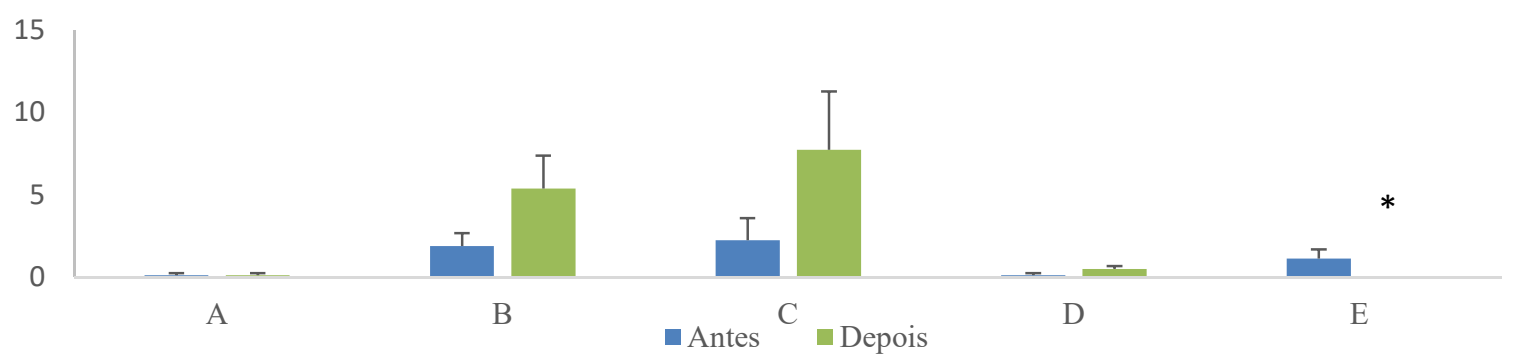

Figura 8: Características sobre a redução de danos. As colunas representam as médias das alternativas: A - Não tem eficácia B - Envolve tratamento medicamentoso; C - Pode envolver uso de droga que cause dependência; D - Tem como objetivo a abstinência, E - Não sei e as barras o EPM. * Indica diferença significativa na alternativa Não sei Antes e Depois. $\mathrm{p}<0,05$, de acordo com o teste $t$ não-pareado de Student. Antes $(\mathrm{n}=289)$ e Depois $(\mathrm{n}=281)$.

\section{Avaliação da comparação entre os cursos do grupo experimental e do grupo controle:}

Os resultados das comparações intergrupos, entre os questionários provenientes $\mathrm{s}$ cursos Ciências Farmacêuticas (diurno e noturno), Enfermagem, Farmácia, Medicina, Nutrição, Odontologia e Psicologia (grupo experimental), Gestão em Saúde Coletiva, Fisioterapia, Fonoaudiologia, Saúde Coletiva e Terapia Ocupacional (grupo controle), foram obtidos pela Análise de Variância de uma via (ANOVA One-Way). O teste post-hoc usado 
para identificar as diferenças foi o teste de Student-Newman-Keuls $(\mathrm{p}<0,05)$. Os dados relativos a cada questão que apresentou diferença significativa são apresentados na sequência.

5. Em relação ao conhecimento sobre o uso de drogas, você se considera (Marque apenas uma alternativa)

\begin{tabular}{l}
\hline Bem informado(a) \\
\hline Mais ou menos informado(a) \\
\hline Mal informado(a)
\end{tabular}

Na Questão 5, a ANOVA One-Way, seguida pelo teste de Student-Newman-Keuls revelou que houve diferença significativa na alternativa Mais ou menos informado(a) nas comparações entre os cursos Fisioterapia $(\bar{X}=33.5 ; \mathrm{EPM}=4.50)$ e Medicina $(\bar{X}=7.25$; EPM $=0,79)$, Fisioterapia $(\bar{X}=33.5 ; \mathrm{EPM}=4.50)$ e Ciências Farmacêuticas diurno $(\bar{X}=25$; EPM $=5)$, Psicologia $(\bar{X}=28.5 ; \mathrm{EPM}=7.50)$ e Medicina $(\bar{X}=7.25 ; \mathrm{EPM}=0,79)$, com $\mathrm{F}_{(12,931)}=$ 4.22 e $\mathrm{p}=0$; Nas alternativas Bem informado $(a) \mathrm{F}_{(12,931)}=2.26$ e $\mathrm{p}=0.07$ e Mal informado(a) $\mathrm{F}_{(12,931)}=2.17$ e $\mathrm{p}=0.09$ não houve diferença significativa (Figura 9).

\section{Conhecimento sobre uso de drogas}

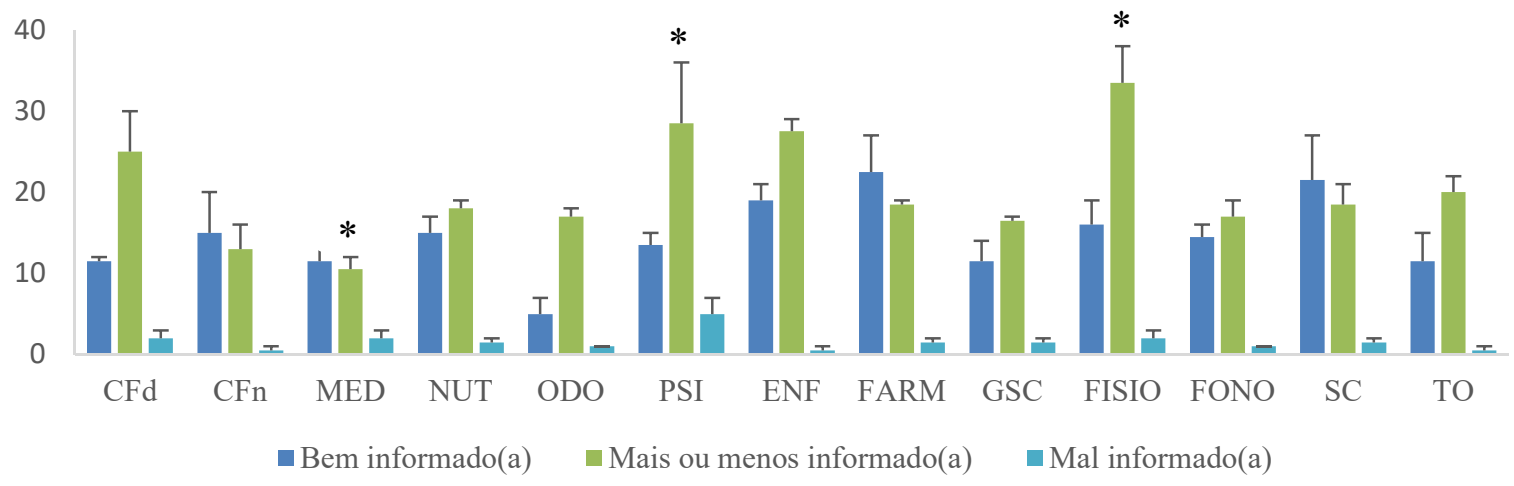

Figura 9: Conhecimento acerca do uso de drogas e da dependência química entre os cursos da área de saúde. As colunas representam as médias e as barras o EPM. $\mathrm{p}<0,05$, de acordo com a ANOVA One-Way. * Indica diferença significativa entre Fisioterapia e Medicina, Fisioterapia e Ciências Farmacêuticas diurno e Psicologia e Medicina, de acordo com o teste de Student-Newman-Keuls $(\mathrm{n}=944)$.

\begin{tabular}{l} 
6. Se você tivesse alguma dúvida a respeito de álcool, maconha ou \\
outras drogas, quem procuraria primeiro? (Marque apenas uma \\
alternativa) \\
\hline Professores \\
\hline Amigos ou colegas de faculdade \\
\hline Pais \\
\hline Serviço de Orientação da UnB \\
\hline Líder religioso (padre, pastor, rabino) \\
\hline
\end{tabular}




\begin{tabular}{l}
\hline Livros e/ou artigos científicos \\
\hline Internet \\
\hline
\end{tabular}

Na Questão 6, a ANOVA One-Way, seguida pelo teste de Student-Newman-Keuls, mostrou que a única alternativa que apresentou diferença significativa foi Livros entre os cursos Psicologia $(\bar{X}=11 ; \mathrm{EPM}=2)$ e Medicina $(\bar{X}=2.50 ; \mathrm{EPM}=2.50)$, Farmácia $(\bar{X}=11$; $\mathrm{EPM}=3)$ e Medicina $(\bar{X}=2.50 ; \mathrm{EPM}=2.50), \operatorname{com} \mathrm{F}_{(12,931)}=3.69$ e $\mathrm{p}=0.01$. Nas demais alternativas, não houve diferença significativa Professores $\mathrm{F}_{(12,931)}=1.21$ e $\mathrm{p}=0.36$, Amigos ou colegas de faculdade $\mathrm{F}_{(12,931)}=1.03$ e $\mathrm{p}=0.47$, Pais $\mathrm{F}_{(12,931)}=1.15$ e $\mathrm{p}=0.40$, Serviço de Orientação da UnB $\mathrm{F}_{(12,931)}=1$ e $\mathrm{p}=0.49$, Religião $\mathrm{F}_{(12,931)}=0.86$ e $\mathrm{p}=0.59$ e Internet $\mathrm{F}_{(12,931)}=1.63$ e $\mathrm{p}=0.19$ (Figura 10).

\section{Quem/o que procuraria primeiro em caso de dúvida sobre o uso de drogas}

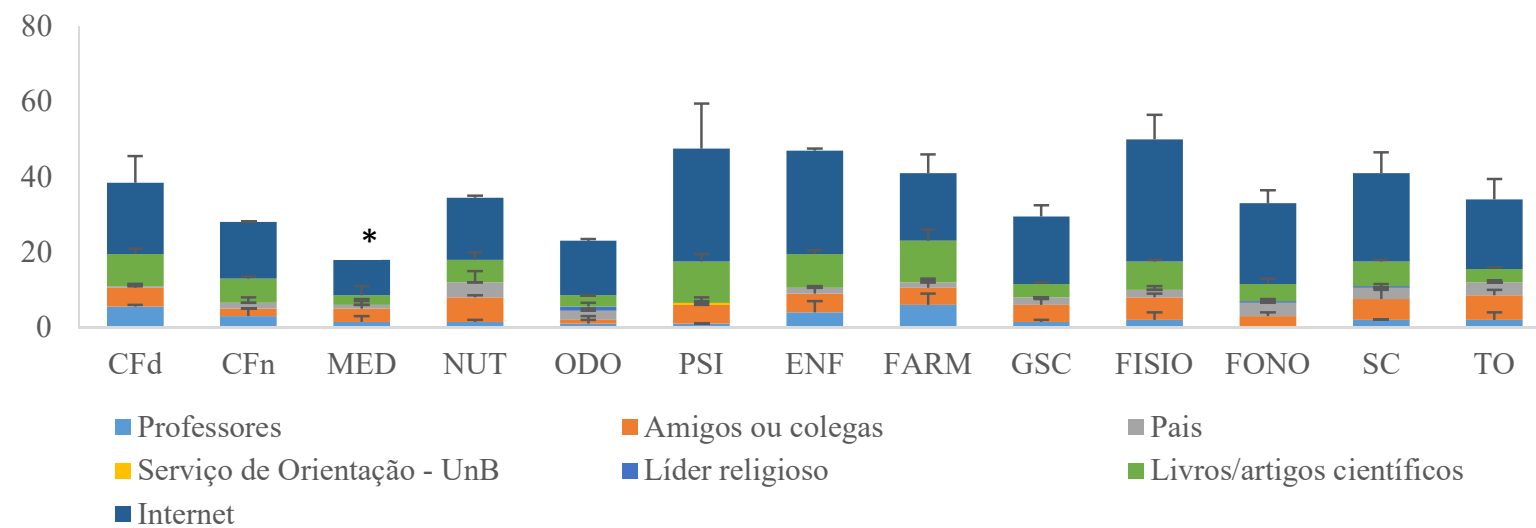

Figura 10: Quem/o que os estudantes procuram primeiro em caso de dúvidas sobre o uso de drogas e dependência química. As colunas representam as médias e as barras o EPM. p < 0,05 , de acordo com a ANOVA One-Way. * Indica diferença significativa entre os cursos Psicologia e Medicina, Farmácia e Medicina na alternativa Livros, de acordo com o teste de Student-Newman-Keuls $(\mathrm{n}=944)$.

\begin{tabular}{l} 
7. O que você pensa a respeito de uma pessoa usar uma única vez \\
as seguintes substâncias? (Marque apenas aprovo, desaprovo ou \\
não sei para cada uma das substâncias) \\
\hline Álcool \\
\hline Benzodiazepínicos e barbitúricos \\
\hline Cigarro de nicotina \\
\hline Cigarro de maconha \\
\hline Cocaína \\
\hline Crack \\
\hline Drogas sintéticas \\
\hline Esteroides anabolizantes \\
\hline Inalantes \\
\hline
\end{tabular}


Na Questão 7, a ANOVA One-Way, seguida pelo teste de Student-Newman-Keuls, revelou que houve diferença significativa nas alternativas Álcool entre os cursos Fisioterapia ( $\bar{X}=40 ; \mathrm{EPM}=0)$ e Odontologia $(\bar{X}=17.5 ; \mathrm{EPM}=3.50)$, Fisioterapia $(\bar{X}=40 ; \mathrm{EPM}=$ $0)$ e Medicina $(\bar{X}=21 ; \mathrm{EPM}=3)$, Psicologia $(\bar{X}=39.5 ; \mathrm{EPM}=5.50)$ e Odontologia $(\bar{X}=$ 17.5; EPM =3.50), Psicologia $(\bar{X}=39.5 ; \mathrm{EPM}=5.50)$ e Medicina $(\bar{X}=21 ; \mathrm{EPM}=3)$ com $\mathrm{F}_{(12,931)}=5.53$ e $\mathrm{p}=0.005 ;$ Cigarro de nicotina entre os cursos Psicologia $(\bar{X}=23 ; \mathrm{EPM}=3)$ e Ciências Farmacêuticas diurno $(\bar{X}=7.50 ; \mathrm{EPM}=2.50)$, Psicologia $(\bar{X}=23 ; \mathrm{EPM}=3)$ e Ciências Farmacêuticas noturno $(\bar{X}=10.50 ; \mathrm{EPM}=5.50)$, Psicologia $(\bar{X}=23 ; \mathrm{EPM}=3) \mathrm{e}$ Medicina $(\bar{X}=7.50 ; \mathrm{EPM}=0.50)$, Psicologia $(\bar{X}=23 ; \mathrm{EPM}=3)$ e Nutrição $(\bar{X}=7 ; \mathrm{EPM}=$ 1), Psicologia $(\bar{X}=23 ; \mathrm{EPM}=3)$ e Odontologia $(\bar{X}=4.50 ; \mathrm{EPM}=2.50)$, Psicologia $(\bar{X}=$ $23 ; \mathrm{EPM}=3)$ e Enfermagem $(\bar{X}=9.50 ; \mathrm{EPM}=0.50)$, Psicologia $(\bar{X}=23 ; \mathrm{EPM}=3) \mathrm{e}$ Farmácia $(\bar{X}=13.50 ;$ EPM $=3.50)$, Psicologia $(\bar{X}=23 ;$ EPM $=3)$ e Gestão em Saúde Coletiva $(\bar{X}=11 ; \mathrm{EPM}=2)$, Psicologia $(\bar{X}=23 ; \mathrm{EPM}=3)$ e Fisioterapia $(\bar{X}=10.50 ; \mathrm{EPM}$ $=2.50)$, Psicologia $(\bar{X}=23 ; \mathrm{EPM}=3)$ e Fonoaudiologia $(\bar{X}=3.50 ; \mathrm{EPM}=0.50)$, Psicologia $(\bar{X}=23 ; \mathrm{EPM}=3)$ e Saúde Coletiva $(\bar{X}=7.50 ; \mathrm{EPM}=0.50)$ e Psicologia $(\bar{X}=23 ; \mathrm{EPM}=$ 3) e Terapia Ocupacional $(\bar{X}=10.50 ; \mathrm{EPM}=0.50)$ com $\mathrm{F}_{(12,931)}=4$ e $\mathrm{p}=0.009 ;$ Cigarro de maconha entre Psicologia $(\bar{X}=27.50 ; \mathrm{EPM}=7.50)$ e Odontologia $(\bar{X}=3.50 ; \mathrm{EPM}=3.50)$, $\operatorname{com} \mathrm{F}_{(12,931)}=2.47$ e $\mathrm{p}=0.05 ;$ Drogas sintéticas entre Psicologia $(\bar{X}=12.5 ; \mathrm{EPM}=1.50) \mathrm{e}$ Nutrição $(\bar{X}=2.5 ; \mathrm{EPM}=1.50)$, Psicologia $(\bar{X}=12.5 ; \mathrm{EPM}=1.50)$ e Odontologia $(\bar{X}=$ 1.50; $\mathrm{EPM}=0.50)$, Psicologia $(\bar{X}=12.5 ; \mathrm{EPM}=1.5)$ e Enfermagem $(\bar{X}=1.50 ; \mathrm{EPM}=$ $0.50)$, Psicologia $(\bar{X}=12.5 ; \mathrm{EPM}=1.50)$ e Fonoaudiologia $(\bar{X}=2.50 ; \mathrm{EPM}=0.50)$, Psicologia $(\bar{X}=12.5 ; \mathrm{EPM}=1.50)$ e Saúde Coletiva $(\bar{X}=3 ; \mathrm{EPM}=2)$, Psicologia $(\bar{X}=$ 12.5; $\mathrm{EPM}=1.50)$ e Terapia Ocupacional $(\bar{X}=3 ; \mathrm{EPM}=1) \operatorname{com} \mathrm{F}_{(12,931)}=2.67$ e p $=0.04$ e Esteroides anabolizantes entre Psicologia $(\bar{X}=6 ; \mathrm{EPM}=2)$ e Terapia Ocupacional $(\bar{X}=$ $0.50 ; \mathrm{EPM}=0.50) \operatorname{com} \mathrm{F}_{(12,931)}=2.73$ e $\mathrm{p}=0.04($ Figura 11$)$. 


\section{Aprovação do uso de substâncias \\ uma única vez}

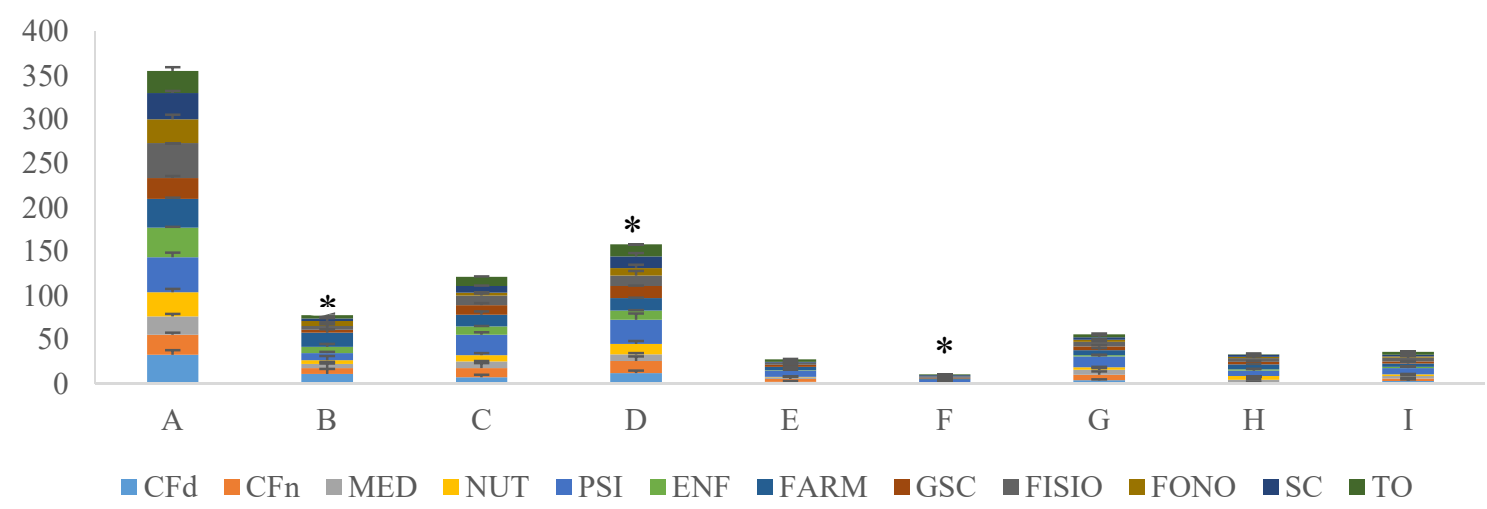

Figura 11: Aprovação do uso uma única vez de substâncias. As colunas representam as médias das alternativas $\mathrm{A}=$ Álcool, $\mathrm{B}=$ Benzodiazepínicos e barbitúricos, $\mathrm{C}=$ Cigarro de nicotina, $\mathrm{D}=$ Cigarro de maconha, $\mathrm{E}=$ Cocaína, $\mathrm{F}=$ Crack, $\mathrm{G}=$ Drogas sintéticas, $\mathrm{H}=$ Esteroides anabolizantes, I = Inalantes e as barras o EPM. e as barras o EPM. p $<0,05$ de acordo com ANOVA One-Way. * Indica diferença significativa de acordo com o teste de Student-Newman-Keuls (vide texto) $(\mathrm{n}=944)$.

Na Questão 7, sobre a desaprovação do uso uma única vez de substâncias, a ANOVA One-Way, seguida pelo teste de Student-Newman-Keuls, houve diferença significativa nas alternativas Cigarro de nicotina entre Fisioterapia $(\bar{X}=36.5$; EPM $=2.50)$ e Ciências Farmacêuticas noturno $(\bar{X}=17 ; \mathrm{EPM}=4)$, Fisioterapia $(\bar{X}=36.5 ; \mathrm{EPM}=2.50)$ e Medicina ( $\bar{X}=14.5 ; \mathrm{EPM}=2.50)$, Fisioterapia $(\bar{X}=36.5 ; \mathrm{EPM}=2.50)$ e Gestão em Saúde Coletiva ( $\bar{X}=16.5 ; \mathrm{EPM}=1.50)$ e Fisioterapia $(\bar{X}=36.5 ; \mathrm{EPM}=2.50)$ e Terapia Ocupacional $(\bar{X}=$ 14.5; $\mathrm{EPM}=7.50), \operatorname{com~}_{(12,931)}=4.44 \mathrm{e} \mathrm{p}=0.006$; Cigarro de maconha entre Fisioterapia ( $\bar{X}=34.5 ; \mathrm{EPM}=5.50)$ e Ciências Farmacêuticas noturno $(\bar{X}=10 ; \mathrm{EPM}=4)$, $\operatorname{com} \mathrm{F}_{(12,931)}=$ 2.68 e p $=0.04$; Cocaína entre Fisioterapia $(\bar{X}=47 ; \mathrm{EPM}=0)$ e Medicina $(\bar{X}=19.5 ; \mathrm{EPM}=$ 2.50), $\operatorname{com} \mathrm{F}_{(12,931)}=2.91$ e $\mathrm{p}=0.03 ;$ Crack entre Fisioterapia $(\bar{X}=48.5 ; \mathrm{EPM}=0.50) \mathrm{e}$ Medicina $(\bar{X}=21.5 ; \mathrm{EPM}=2.50)$ e Fisioterapia $(\bar{X}=48.5 ; \mathrm{EPM}=0.50)$ e Terapia Ocupacional $(\bar{X}=22.5 ; \mathrm{EPM}=9.50), \operatorname{com}_{(12,931)}=3.72$ e $\mathrm{p}=0.01 ;$ Drogas sintéticas entre os cursos Fisioterapia $(\bar{X}=39 ; \mathrm{EPM}=1)$ e Ciências Farmacêuticas noturno $(\bar{X}=17.5$; EPM $=2.50)$, Fisioterapia $(\bar{X}=39 ; \mathrm{EPM}=1)$ e Medicina $(\bar{X}=15.5 ; \mathrm{EPM}=1.50)$, Fisioterapia $(\bar{X}$ $=39 ; \mathrm{EPM}=1)$ e Psicologia $(\bar{X}=22 ; \mathrm{EPM}=3)$, Fisioterapia $(\bar{X}=39 ; \mathrm{EPM}=1)$ e Gestão 
em Saúde Coletiva $(\bar{X}=18.5 ; \mathrm{EPM}=3.50)$, Fisioterapia $(\bar{X}=39 ; \mathrm{EPM}=1)$ e Terapia Ocupacional $(\bar{X}=19 ; \mathrm{EPM}=6), \operatorname{com}_{(12,931)}=5.78$ e $\mathrm{p}=0.001$ e Inalantes entres os cursos Enfermagem $(\bar{X}=37.5 ; \mathrm{EPM}=1.50)$ e Medicina $(\bar{X}=19 ; \mathrm{EPM}=2)$, Enfermagem $(\bar{X}=$ 37.5; EPM $=1.50)$ e Terapia Ocupacional $(\bar{X}=19 ; \mathrm{EPM}=7)$, Fisioterapia $(\bar{X}=41 ; \mathrm{EPM}=$ 2) e Ciências Farmacêuticas noturno $(\bar{X}=21.5$; EPM $=1.50)$, Fisioterapia $(\bar{X}=41$; EPM $=$ 2) e Medicina $(\bar{X}=19 ; \mathrm{EPM}=2)$, Fisioterapia $(\bar{X}=41 ; \mathrm{EPM}=2)$ e Gestão em Saúde Coletiva $(\bar{X}=22 ; \mathrm{EPM}=2)$, Fisioterapia $(\bar{X}=41 ; \mathrm{EPM}=2)$ e Terapia Ocupacional $(\bar{X}=$ 19; EPM =76), Saúde Coletiva $(\bar{X}=42 ; E P M=0)$ e Ciências Farmacêuticas noturno $(\bar{X}=$ 21.5; EPM = 1.50), Saúde Coletiva $(\bar{X}=42 ; \mathrm{EPM}=0)$ e Medicina $(\bar{X}=19 ; \mathrm{EPM}=2)$, Saúde Coletiva $(\bar{X}=42 ; \mathrm{EPM}=0)$ e Gestão em Saúde Coletiva $(\bar{X}=22 ; \mathrm{EPM}=2)$, Saúde Coletiva $(\bar{X}=42 ; \mathrm{EPM}=)$ e Terapia Ocupacional $(\bar{X}=19 ; \mathrm{EPM}=7), \operatorname{com} \mathrm{F}_{(12,931)}=5.75 \mathrm{e}$ $\mathrm{p}=0.001$. Contudo, não houve diferença significativa nas alternativas Álcool com $\mathrm{F}_{(12,931)}=$ 1.48 e $\mathrm{p}=0.24$; Benzodiazepínicos e barbitúricos $\operatorname{com} \mathrm{F}_{(12,931)}=0.80$ e $\mathrm{p}=0.64$ e Esteroides anabolizantes com $\mathrm{F}_{(12,931)}=2.41$ e $\mathrm{p}=0.06$ (Figura 12).

\section{Desaprovação do uso de substâncias uma única vez}

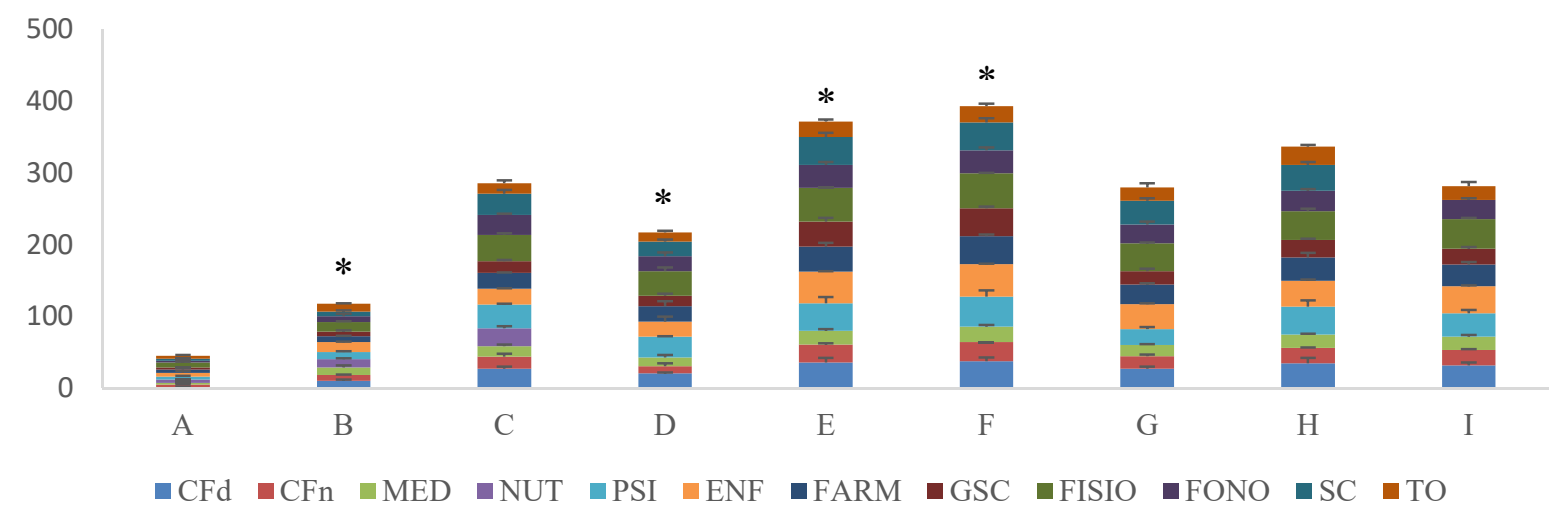

Figura 12: Desaprovação do uso de substâncias uma única vez. As colunas representam as médias das alternativas $\mathrm{A}=$ Álcool, $\mathrm{B}=$ Benzodiazepínicos e barbitúricos, $\mathrm{C}=$ Cigarro de nicotina, $\mathrm{D}=$ Cigarro de maconha, $\mathrm{E}=$ Cocaína, $\mathrm{F}=\mathrm{Crack}, \mathrm{G}=$ Drogas sintéticas, $\mathrm{H}=$ Esteroides anabolizantes, $\mathrm{I}=$ Inalantes e as barras o EPM. $\mathrm{p}<0,05$, de acordo com a ANOVA One-Way. * Indica diferença significativa de acordo com o teste de Student-Newman-Keuls (vide texto) $(\mathrm{n}=944)$. 
Na Questão 7, na alternativa não sei sobre o uso uma única vez de substâncias, a ANOVA One-Way, seguida pelo teste de Student-Newman-Keuls, revelou que houve diferença significativa nas alternativas Álcool entre os cursos Enfermagem ( $\bar{X}=7.50$; $\mathrm{EPM}=2.50)$ e Ciências Farmacêuticas diurno $(\bar{X}=2.50 ; \mathrm{EPM}=0.50)$, Enfermagem $(\bar{X}=$ 7.50; EPM $=2.50)$ e Ciências Farmacêuticas noturno $(\bar{X}=2.50 ; \mathrm{EPM}=0.50)$, Enfermagem ( $\bar{X}=7.50 ; \mathrm{EPM}=2.50)$ e Medicina $(\bar{X}=1 ; \mathrm{EPM}=0)$, Enfermagem $(\bar{X}=7.50 ; \mathrm{EPM}=2.50)$ e Nutrição $(\bar{X}=2 ; E P M=0)$, Enfermagem $(\bar{X}=7.50 ; E P M=2.50)$ e Odontologia $(\bar{X}=$ 2.50; $\mathrm{EPM}=0.50)$, Enfermagem $(\bar{X}=7.50 ; \mathrm{EPM}=2.50)$ e Psicologia $(\bar{X}=3 ; \mathrm{EPM}=0)$, Enfermagem $(\bar{X}=7.50 ; \mathrm{EPM}=2.50)$ e Gestão em Saúde Coletiva $(\bar{X}=3 ; \mathrm{EPM}=1)$, Enfermagem $(\bar{X}=7.50 ; \mathrm{EPM}=2.50)$ e Fonoaudiologia $(\bar{X}=3.50 ; \mathrm{EPM}=0.50)$, Enfermagem $(\bar{X}=7.50 ; \mathrm{EPM}=2.50)$ e Saúde Coletiva $(\bar{X}=2 ; \mathrm{EPM}=0)$, Enfermagem $(\bar{X}$ $=7.50 ; \mathrm{EPM}=2.50)$ e Terapia Ocupacional $(\bar{X}=1 ; \mathrm{EPM}=0) \operatorname{com} \mathrm{F}_{(12,931)}=3.28$ e p $=0.02$ e Cigarro de nicotina Farmácia $(\bar{X}=6 ; \mathrm{EPM}=1)$ e Ciências Farmacêuticas noturno $(\bar{X}=1$; $\mathrm{EPM}=50)$, com $\mathrm{F}_{(12,931)}=3.48 \mathrm{e} \mathrm{p}=0.02$. As demais alternativas não apresentaram diferença significativa com Benzodiazepínicos e barbitúricos com $\mathrm{F}_{(12,931)}=2.44$ e $\mathrm{p}=0.06$, Cigarro de maconha $\operatorname{com} \mathrm{F}_{(12,931)}=1.52$ e $\mathrm{p}=0.23$, Cocaína $\operatorname{com} \mathrm{F}_{(12,931)}=1.90$ e $\mathrm{p}=0.13$, Crack $\mathrm{com}$ $\mathrm{F}_{(12,931)}=2.40$ e $\mathrm{p}=0.06$, Drogas sintéticas com $\mathrm{F}_{(12,931)}=2.12$ e $\mathrm{p}=0.09$, Esteroides anabolizantes com $\mathrm{F}_{(12,931)}=1.70$ e $\mathrm{p}=0.17$ e Inalantes $\operatorname{com} \mathrm{F}_{(12,931)}=1.37$ e $\mathrm{p}=0.29$ (Figura $13)$. 


\section{Não sabem opinar sobre o uso de substâncias uma única vez}

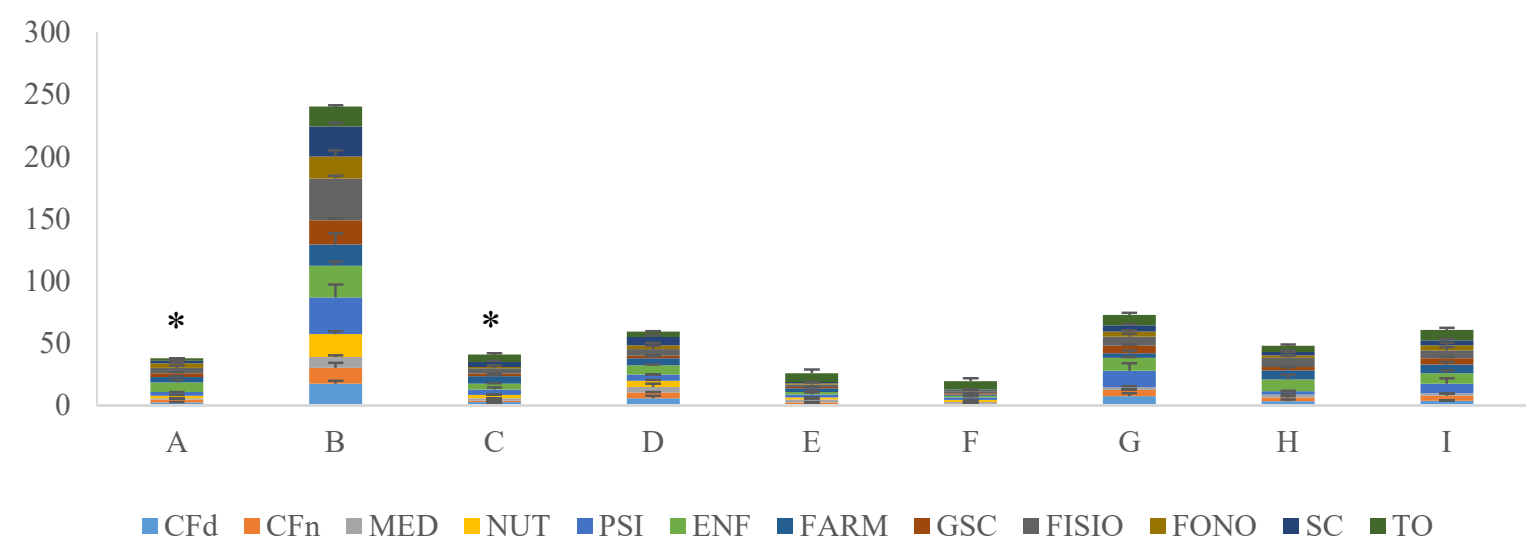

Figura 13: Alternativa não sei sobre o uso de substâncias uma única vez. As colunas representam as médias das alternativas $\mathrm{A}=$ Álcool, $\mathrm{B}=$ Benzodiazepínicos e barbitúricos, $\mathrm{C}=$ Cigarro de nicotina, $\mathrm{D}=$ Cigarro de maconha, $\mathrm{E}=$ Cocaína, $\mathrm{F}=$ Crack, $\mathrm{G}=$ Drogas sintéticas, $\mathrm{H}=$ Esteroides anabolizantes, $\mathrm{I}=$ Inalantes e as barras o EPM. $\mathrm{p}<0,05$, de acordo com a ANOVA One-Way. * Indica diferença significativa de acordo com o teste de StudentNewman-Keuls (vide texto) $(\mathrm{n}=944)$.

\begin{tabular}{l} 
8. O que você pensa a respeito de uma pessoa usar com \\
frequência as seguintes substâncias? (Marque apenas aprovo, \\
desaprovo ou não sei para cada uma das substâncias) \\
\hline Álcool \\
\hline Benzodiazepínicos e barbitúricos \\
\hline Cigarro de nicotina \\
\hline Cigarro de maconha \\
\hline Cocaína \\
\hline Crack \\
\hline Drogas sintéticas \\
\hline Esteroides anabolizantes \\
\hline Inalantes
\end{tabular}

Na Questão 8, a ANOVA One-Way, seguida pelo teste de Student-Newman-Keuls revelou que houve diferença significativa apenas na alternativa Cigarro de nicotina entre os cursos Psicologia $(\bar{X}=6.5 ; \mathrm{EPM}=0.50)$ e Medicina $(\bar{X}=0 ; \mathrm{EPM}=0)$, Psicologia $(\bar{X}=6.5$; $\mathrm{EPM}=0.50)$ e Nutrição $(\bar{X}=1 ; \mathrm{EPM}=1)$, Psicologia $(\bar{X}=6.5 ; \mathrm{EPM}=0.50)$ e Odontologia $(\bar{X}=0 ; \mathrm{EPM}=0)$, Psicologia $(\bar{X}=6.5 ; \mathrm{EPM}=0.5)$ e Enfermagem $(\bar{X}=1.50 ; \mathrm{EPM}=0.50)$, Psicologia $(\bar{X}=6.5 ; \mathrm{EPM}=0.50)$ e Fonoaudiologia $(\bar{X}=1 ; \mathrm{EPM}=0)$, Psicologia $(\bar{X}=6.5$; $\mathrm{EPM}=0.50)$ e Saúde Coletiva $(\bar{X}=2.50 ; \mathrm{EPM}=1.50)$, Psicologia $(\bar{X}=6.5 ; \mathrm{EPM}=0.50)$ e Terapia Ocupacional $(\bar{X}=1.50 ; \mathrm{EPM}=0.50)$, Farmácia $(\bar{X}=6 ; \mathrm{EPM}=1)$ e Medicina $(\bar{X}=$ 
$0 ; \mathrm{EPM}=0)$, Farmácia $(\bar{X}=6 ; \mathrm{EPM}=1)$ e Odontologia $(\bar{X}=0 ; \mathrm{EPM}=0)$, com $\mathrm{F}_{(12,931)}=$ 4.45 e $\mathrm{p}=0.06$. As demais alternativas não apresentaram diferença significativa: Álcool $\mathrm{F}_{(12,931)}=2$ e $\mathrm{p}=0.11 ;$ Benzodiazepínicos e barbitúricos $\mathrm{F}_{(12,931)}=1.29$ e $\mathrm{p}=0.32 ;$ Maconha $\mathrm{F}_{(12,931)}=2.44$ e $\mathrm{p}=0.06 ;$ Cocaína $\mathrm{F}_{(12,931)}=0.84$ e $\mathrm{p}=0.06 ;$ Crack $\mathrm{F}_{(12,931)}=1.64$ e $\mathrm{p}=0.19$; Drogas sintéticas $\mathrm{F}_{(12,931)}=0.89$ e $\mathrm{p}=0.57$; Esteroides anabolizantes $\mathrm{F}_{(12,931)}=1.02$ e $\mathrm{p}=$ 0.48 e Inalantes $\mathrm{F}_{(12,931)}=1.11$ e $\mathrm{p}=0.42$ (Figura 14).

\section{Aprovação do uso frequente de substâncias}

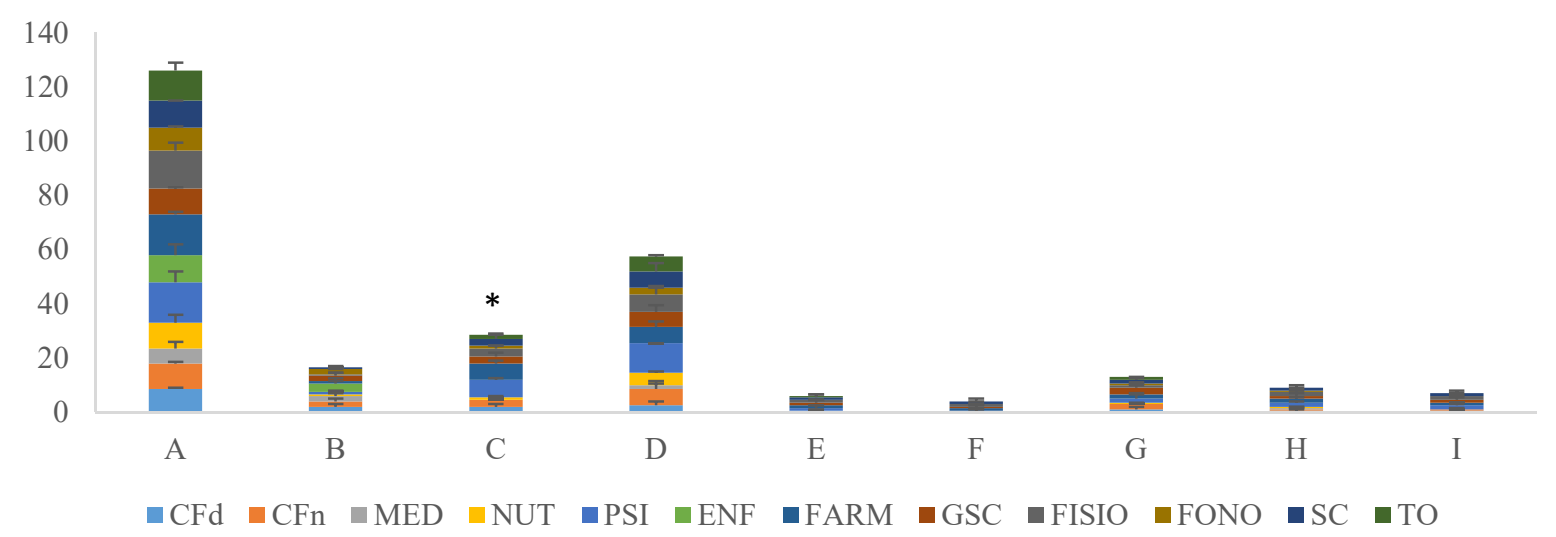

Figura 14: Aprovação do uso frequente de substâncias. As colunas representam as médias das alternativas $\mathrm{A}=$ Álcool, $\mathrm{B}=$ Benzodiazepínicos e barbitúricos, $\mathrm{C}=$ Cigarro de nicotina, $\mathrm{D}=$ Cigarro de maconha, $\mathrm{E}=$ Cocaína, $\mathrm{F}=\mathrm{Crack}, \mathrm{G}=$ Drogas sintéticas, $\mathrm{H}=$ Esteroides anabolizantes, $\mathrm{I}=$ Inalantes e as barras o EPM. $\mathrm{p}<0,05$, de acordo com a ANOVA One-Way. * Indica diferença significativa de acordo com o teste de Student-Newman-Keuls (vide texto) $(\mathrm{n}=944)$.

Ainda na Questão 8, a respeito da desaprovação do uso frequente de substâncias, a ANOVA One-Way, seguida pelo teste de Student-Newman-Keuls, mostrou que houve diferença significativa nas alternativas Cigarro de nicotina entre os cursos Fisioterapia $(\bar{X}=$ 47.5; $\mathrm{EPM}=1.50)$ e Medicina $(\bar{X}=21.5 ; \mathrm{EPM}=3.50)$, Fisioterapia $(\bar{X}=47.5 ; \mathrm{EPM}=1.50)$ e Odontologia $(\bar{X}=22.5 ; \mathrm{EPM}=2.50)$, com $\mathrm{F}_{(12,931)}=3.62$ e $\mathrm{p}=0.01 ;$ Cigarro de maconha entre os cursos Fisioterapia $(\bar{X}=43.5 ; \mathrm{EPM}=0.50)$ e Ciências Farmacêuticas noturno $(\bar{X}=$ $20.5 ; \mathrm{EPM}=1.50)$, Fisioterapia $(\bar{X}=43.5 ; \mathrm{EPM}=0.50)$ e Medicina $(\bar{X}=18.5 ; \mathrm{EPM}=$ 3.50), Fisioterapia $(\bar{X}=43.5 ; \mathrm{EPM}=0.50)$ e Odontologia $(\bar{X}=21.5 ; \mathrm{EPM}=1.50)$, Fisioterapia $(\bar{X}=43.5 ; \mathrm{EPM}=0.50)$ e Gestão em Saúde Coletiva $(\bar{X}=20.5 ; \mathrm{EPM}=5.50)$, 
Fisioterapia $(\bar{X}=43.5 ; \mathrm{EPM}=0.50)$ e Terapia Ocupacional $(\bar{X}=20 ; \mathrm{EPM}=6)$, com $\mathrm{F}_{(12,931)}$ $=3.91$ e p $=0.01 ;$ Cocaína entre os cursos Fisioterapia $(\bar{X}=50 ;$ EPM $=2)$ e Medicina $(\bar{X}=$ 23; $\mathrm{EPM}=3)$, Fisioterapia $(\bar{X}=50 ; \mathrm{EPM}=2)$ e Odontologia $(\bar{X}=22.5 ; \mathrm{EPM}=2.50)$, com $\mathrm{F}_{(12,931)}=3.46$ e $\mathrm{p}=0.01$, Crack entre os cursos Fisioterapia $(\bar{X}=50 ; \mathrm{EPM}=2)$ e Medicina ( $\bar{X}=23 ; \mathrm{EPM}=3)$, Fisioterapia $(\bar{X}=50 ; \mathrm{EPM}=2)$ e Odontologia $(\bar{X}=22.5 ; \mathrm{EPM}=2.50)$, com $\mathrm{F}_{(12,931)}=3.70$ e $\mathrm{p}=0.01$, Drogas sintéticas entre os cursos Fisioterapia $(\bar{X}=45.5 ; \mathrm{EPM}$ $=2.50)$ e Medicina $(\bar{X}=20.5 ; \mathrm{EPM}=2.50)$, Fisioterapia $(\bar{X}=45.5 ; \mathrm{EPM}=2.50) \mathrm{e}$

Odontologia $(\bar{X}=21.5 ; \mathrm{EPM}=2.50)$, com $\mathrm{F}_{(12,931)}=3.76$ e $\mathrm{p}=0.01 ;$ Esteroides anabolizantes entre os cursos Fisioterapia $(\bar{X}=46 ; \mathrm{EPM}=1)$ e Medicina $(\bar{X}=21 ; \mathrm{EPM}=)$, com $\mathrm{F}_{(12,931)}=3.19$ e p $=0.02$ e Inalantes entre os cursos Gestão em Saúde Coletiva $(\bar{X}=$ 46.5; $\mathrm{EPM}=0.5)$ e Medicina $(\bar{X}=21.5 ; \mathrm{EPM}=2.50)$, Gestão em Saúde Coletiva $(\bar{X}=46.5 ;$ $\mathrm{EPM}=0.50)$ e Odontologia $(\bar{X}=22 ; \mathrm{EPM}=2), \operatorname{com} \mathrm{F}_{(12,931)}=3.34$ e $\mathrm{p}=0.02$. Não houve diferença nas alternativas Álcool com $\mathrm{F}_{(12,931)}=2.21$ e p $=0.08$ e Benzodiazepínicos e barbitúricos $\operatorname{com} \mathrm{F}_{(12,931)}=1.36$ e $\mathrm{p}=0.29$ (Figura 15).

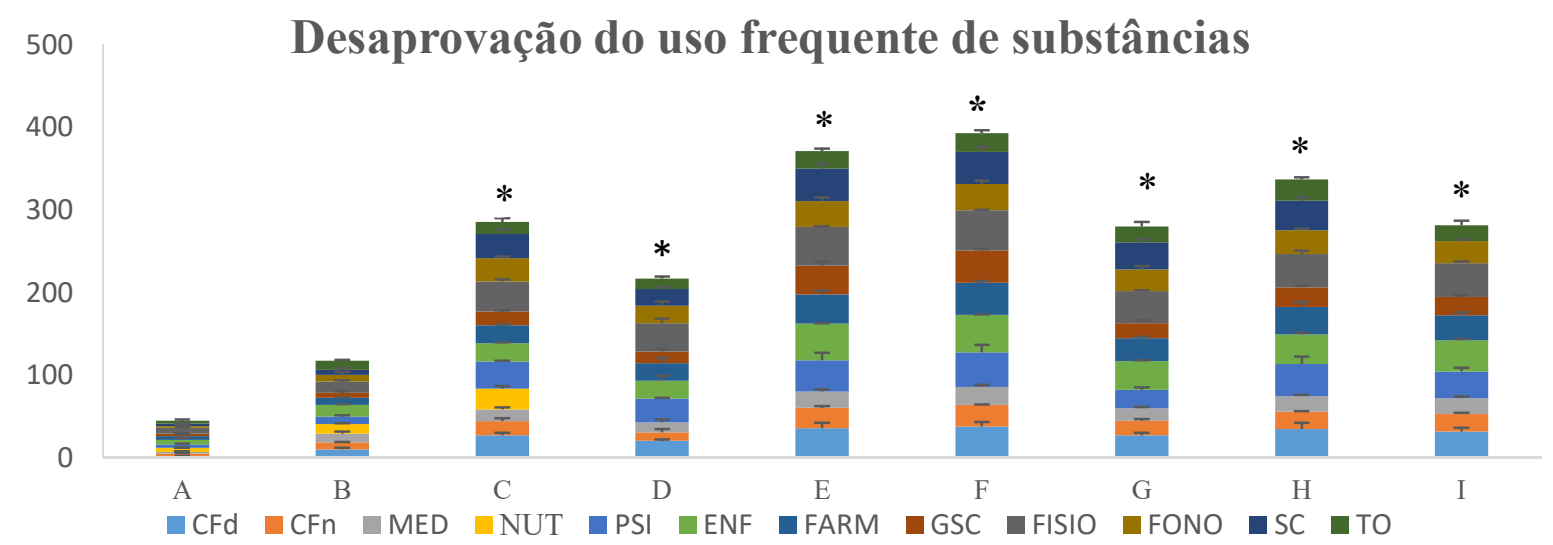

Figura 15: Desaprovação do uso frequente de substâncias. As colunas representam as médias das alternativas $\mathrm{A}=$ Álcool, $\mathrm{B}=$ Benzodiazepínicos e barbitúricos, $\mathrm{C}=$ Cigarro de nicotina, $\mathrm{D}$ $=$ Cigarro de maconha, $\mathrm{E}=$ Cocaína, $\mathrm{F}=\mathrm{Crack}, \mathrm{G}=$ Drogas sintéticas, $\mathrm{H}=$ Esteroides anabolizantes, $\mathrm{I}=$ Inalantes e as barras o EPM. $\mathrm{p}<0,05$ de acordo com a ANOVA One-Way. * Indica diferença significativa de acordo com o teste de Student-Newman-Keuls (vide texto) $(\mathrm{n}=944)$.

Finalmente, na Questão 8, a alternativa não sei sobre o uso frequente de substâncias, a ANOVA One-Way, seguida pelo teste de Student-Newman-Keuls, apresentou diferença 
significativa apenas na alternativa Drogas sintéticas entre os cursos: Psicologia $(\bar{X}=9.50$; $\mathrm{EPM}=2.50)$ e Ciências Farmacêuticas noturno $(\bar{X}=1 ; \mathrm{EPM}=1)$, Psicologia $(\bar{X}=9.50$; $\mathrm{EPM}=2.50)$ e Medicina $(\bar{X}=3.50 ; \mathrm{EPM}=0.50)$, Psicologia $(\bar{X}=9.50 ; \mathrm{EPM}=2.50) \mathrm{e}$ Nutrição $(\bar{X}=3 ; \mathrm{EPM}=2)$, Psicologia $(\bar{X}=9.50 ; \mathrm{EPM}=2.50)$ e Odontologia $(\bar{X}=0.50$; $\mathrm{EPM}=0.50)$, Psicologia $(\bar{X}=9.50 ; \mathrm{EPM}=2.50)$ e Farmácia $(\bar{X}=2 ; \mathrm{EPM}=1)$, Psicologia ( $\bar{X}=9.50 ; \mathrm{EPM}=2.50)$ e Gestão em Saúde Coletiva $(\bar{X}=2.50 ; \mathrm{EPM}=0.50)$, Psicologia $(\bar{X}$ $=9.50 ; \mathrm{EPM}=2.50)$ e Fonoaudiologia $(\bar{X}=0.50 ; \mathrm{EPM}=0.50)$, Psicologia $(\bar{X}=9.50 ; \mathrm{EPM}$ $=2.50)$ e Saúde Coletiva $(\bar{X}=3 ; E P M=2), \operatorname{com}_{(12,931)}=3.38$ e $\mathrm{p}=0.001$. As outras alternativas não apresentaram diferença significativa: Álcool $\mathrm{F}_{(12,931)}=2.25$ e p $=0.08$; Benzodiazepínicos e barbitúricos $\mathrm{F}_{(12,931)}=1.73$ e $\mathrm{p}=0.16$; Cigarro de nicotina $\mathrm{F}_{(12,931)}=$ 2.43 e $\mathrm{p}=0.06 ;$ Cigarro de maconha $\mathrm{F}_{(12,931)}=1.40$ e $\mathrm{p}=0.27$; Cocaína $\mathrm{F}_{(12,931)}=0.83$ e $\mathrm{p}=$ $0.62 ;$ Crack $\mathrm{F}_{(12,931)}=0.61$ e $\mathrm{p}=0.79 ;$ Esteroides anabolizantes $\mathrm{F}_{(12,931)}=0.61$ e $\mathrm{p}=0.79$ e Inalantes $\mathrm{F}_{(12,931)}=1.57$ e $\mathrm{p}=0.21$ (Figura 16).

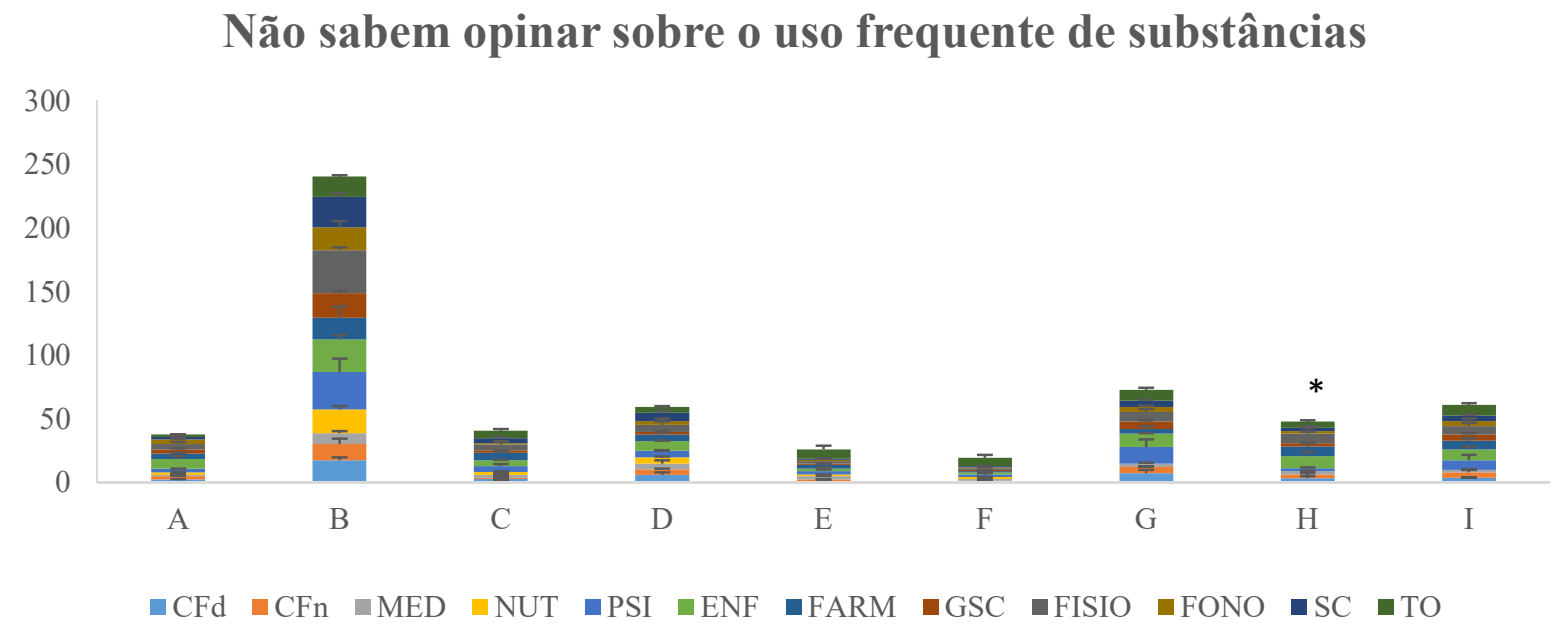

Figura 16: Alternativa não sei sobre usar frequentemente substâncias. As colunas representam as médias das alternativas $\mathrm{A}=$ Álcool, $\mathrm{B}=$ Benzodiazepínicos e barbitúricos, $\mathrm{C}=$ Cigarro de nicotina, $\mathrm{D}=$ Cigarro de maconha, $\mathrm{E}=$ Cocaína, $\mathrm{F}=\mathrm{Crack}, \mathrm{G}=$ Drogas sintéticas, $\mathrm{H}=$ Esteroides anabolizantes, $\mathrm{I}=$ Inalantes e as barras o EPM. $\mathrm{p}<0,05$, de acordo com a ANOVA One-Way. * Indica diferença significativa de acordo com o teste de StudentNewman-Keuls (vide texto) $(\mathrm{n}=944)$.

\begin{tabular}{l}
\hline $\begin{array}{l}\text { 9. Para você, existe diferença entre usuário de drogas e } \\
\text { dependente químico? }\end{array}$ \\
\hline Sim \\
\hline Não
\end{tabular}


Na Questão 9, a ANOVA One-Way, seguida pelo teste de Student-Newman- Keuls, revelou que houve diferença significativa na alternativa Sim entre os cursos Psicologia ${ }^{\bar{X}}=$ 39.50; EPM = 5.50) e Ciências Farmacêuticas noturno ( $\bar{X}=20 ; \mathrm{EPM}=5)$, Psicologia $(\bar{X}$ $=39.50 ; \mathrm{EPM}=5.50)$ e Medicina $(\bar{X}=18 ; \mathrm{EPM}=2)$, Psicologia $(\bar{X}=39.50 ; \mathrm{EPM}=5.50) \mathrm{e}$ Odontologia $(\bar{X}=13.50 ; \mathrm{EPM}=2.50)$, Enfermagem $(\bar{X}=33 ; \mathrm{EPM}=0)$ e Odontologia $(\bar{X}=$ 13.50; $\mathrm{EPM}=2.50)$, Fisioterapia $(\bar{X}=33.50 ; \mathrm{EPM}=1.50)$ e Odontologia $(\bar{X}=13.50 ; \mathrm{EPM}$ $=2.50)$, com $\mathrm{F}_{(12,931)}=4.27$ e $\mathrm{p}=0.007$. Alternativa $N a ̃ o$ sem diferença significativa $\mathrm{F}_{(12,931)}=$ 2.08 e $\mathrm{p}=0.10$ (Figura 17).

\section{Diferença entre usuário e dependente químico}

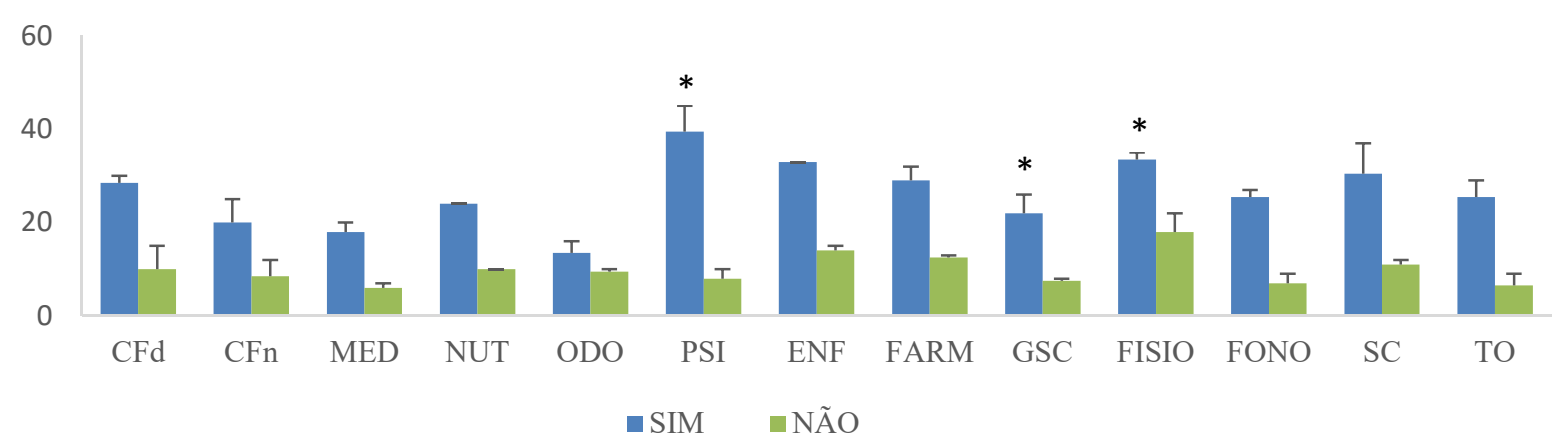

Figura 17: Diferença entre usuário de drogas e dependente químico. As colunas representam as médias e as barras o EPM. $\mathrm{p}<0,05$, de acordo com a ANOVA One-Way. * Indica diferença significativa de acordo com o teste de Student-Newman-Keuls (vide texto) ( $\mathrm{n}=$ 944).

\begin{tabular}{l} 
10. Como você vê a dependência química? (Assinale todas as \\
alternativas que se aplicam) \\
\hline Indica fraqueza moral \\
\hline Mostra a falta de vontade de mudar \\
\hline Pode ser combatida pela fé \\
\hline Tem cura \\
\hline Tem tratamento \\
\hline Nenhuma das anteriores \\
\hline
\end{tabular}

Na Questão 10, a ANOVA One-Way, seguida pelo teste de Student-Newman-Keuls, revelou que houve diferença significativa nas alternativas Indica fraqueza moral entre os cursos Fisioterapia $(\bar{X}=22 ; \mathrm{EPM}=1)$ e Psicologia $(\bar{X}=14.5 ; \mathrm{EPM}=0.50), \operatorname{com} \mathrm{F}_{(12,931)}=$ 3.05 e $\mathrm{p}=0.02 ;$ Tem cura entre os cursos Fisioterapia $(\bar{X}=22 ; \mathrm{EPM}=1)$ e Terapia Ocupacional $(\bar{X}=4.50 ; E P M=2.50)$, Enfermagem $(\bar{X}=21 ; E P M=0)$ e Terapia 
Ocupacional $(\bar{X}=4.50 ; \mathrm{EPM}=2.50)$, com $\mathrm{F}_{(12,931)}=3.46$ e $\mathrm{p}=0.01 ;$ Tem tratamento entre os cursos Fisioterapia $(\bar{X}=46.5 ; \mathrm{EPM}=1)$ e Medicina $(\bar{X}=20.5 ; \mathrm{EPM}=0.50)$, Fisioterapia $(\bar{X}=46.5 ; \mathrm{EPM}=3.50)$ e Odontologia $(\bar{X}=19 ; \mathrm{EPM}=1)$, Psicologia $(\bar{X}=45 ; \mathrm{EPM}=8) \mathrm{e}$ Medicina $(\bar{X}=20.5 ; \mathrm{EPM}=0.50)$ e Psicologia $(\bar{X}=45 ; \mathrm{EPM}=8)$ e Odontologia $(\bar{X}=19 ;$ $\mathrm{EPM}=1)$, com $\mathrm{F}_{(12,931)}=4.33$ e $\mathrm{p}=0.006 ;$ Nenhum dos tratamentos anteriores entre os cursos Gestão em Saúde Coletiva $(\bar{X}=5.50 ; \mathrm{EPM}=0.50)$ e Ciências Farmacêuticas noturno $(\bar{X}=1 ; E P M=1)$, Gestão em Saúde Coletiva $(\bar{X}=5.50 ;$ EPM $=0.50)$ e Nutrição $(\bar{X}=1 ;$ $\mathrm{EPM}=0)$, Gestão em Saúde Coletiva $(\bar{X}=5.50 ; \mathrm{EPM}=0.50)$ e Farmácia $(\bar{X}=0.50 ; \mathrm{EPM}=$ 0.50), Gestão em Saúde Coletiva $(\bar{X}=5.50 ;$ EPM $=0.50)$ e Fisioterapia $(\bar{X}=0.50 ;$ EPM $=$ $0.50)$, Gestão em Saúde Coletiva $(\bar{X}=5.50 ; \mathrm{EPM}=0.50)$ e Fonoaudiologia $(\bar{X}=0 ; \mathrm{EPM}=$ 0), Gestão em Saúde Coletiva $(\bar{X}=5.50 ; \mathrm{EPM}=0.50)$ e Saúde Coletiva $(\bar{X}=1 ; \mathrm{EPM}=1)$, Gestão em Saúde Coletiva $(\bar{X}=5.50 ; \mathrm{EPM}=0.50)$ e Terapia Ocupacional $(\bar{X}=0.50 ; \mathrm{EPM}$ $=0.50), \operatorname{com} \mathrm{F}_{(12,931)}=2.91$ e $\mathrm{p}=0.03($ Figura 18).

\section{Justificativas sobre a dependência química}

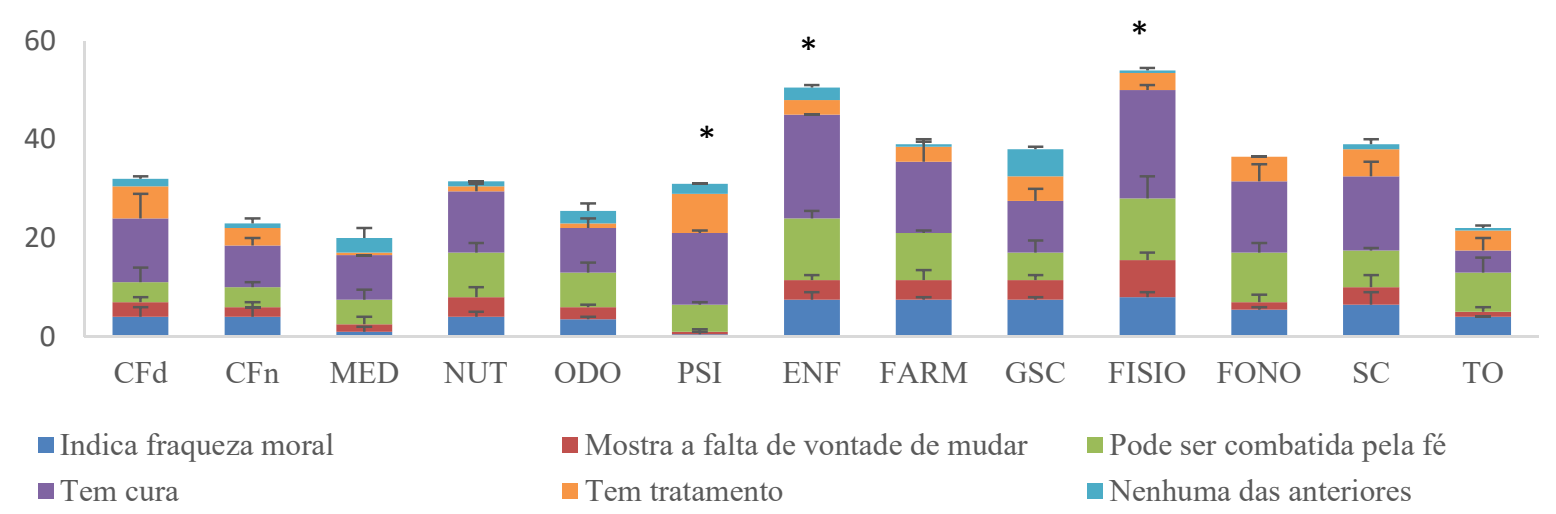

Figura 18: Justificativas sobre a dependência química. As colunas representam as médias e as barras o EPM. p < 0,05, de acordo com a ANOVA One-Way. * Indica diferença significativa de acordo com o teste de Student-Newman-Keuls (vide texto) $(\mathrm{n}=944)$.

\begin{tabular}{l}
$\begin{array}{l}\text { 11. O que poderia caracterizar a dependência química? (Assinale } \\
\text { todas as alternativas que se aplicam) }\end{array}$ \\
\hline Abandono dos prazeres e interesses \\
\hline Estado de abstinência \\
\hline Dificuldade de controlar o consumo \\
\hline Fissura \\
\hline Persistência no uso \\
\hline
\end{tabular}




\begin{tabular}{l}
\hline Tolerância \\
\hline Nenhuma das anteriores \\
\hline
\end{tabular}

Na Questão 11, a ANOVA One-Way, seguida pelo teste de Student-Newman- Keuls, revelou que houve diferença significativa na alternativa Dificuldade de controlar o consumo entre os cursos Fisioterapia $(\bar{X}=46 ; \mathrm{EPM}=1)$ e Odontologia $(\bar{X}=18 ; \mathrm{EPM}=2)$,

Fisioterapia $(\bar{X}=46 ; \mathrm{EPM}=1)$ e Medicina $(\bar{X}=20 ; \mathrm{EPM}=1)$, Psicologia $(\bar{X}=46 ; \mathrm{EPM}=$ 8) e Odontologia $(\bar{X}=18 ; \mathrm{EPM}=2)$, Psicologia $(\bar{X}=46 ; \mathrm{EPM}=8)$ e Medicina $(\bar{X}=20$; $\mathrm{EPM}=1)$, Enfermagem $(\bar{X}=42.5 ; \mathrm{EPM}=0.50)$ e Odontologia $(\bar{X}=18 ; \mathrm{EPM}=2)$, com $\mathrm{F}_{(12,931)}=4.54$ e $\mathrm{p}=0.005$. As demais alternativas não apresentaram diferença significativa Abandono dos prazeres $\mathrm{F}_{(12,931)}=1.51 \mathrm{e} \mathrm{p}=0.40$; Estado de abstinência com $\mathrm{F}_{(12,931)}=2.29 \mathrm{e}$ $\mathrm{p}=0.07 ;$ Fissura $\mathrm{F}_{(12,931)}=2.34$ e $\mathrm{p}=0.07 ;$ Tolerância $\mathrm{F}_{(12,931)}=1.31$ e $\mathrm{p}=0.31$ e Nenhuma das anteriores $\mathrm{F}_{(12,931)}=1.20 \mathrm{e} \mathrm{p}=0.37$ (Figura 19).

\section{Caracterização da dependência química}

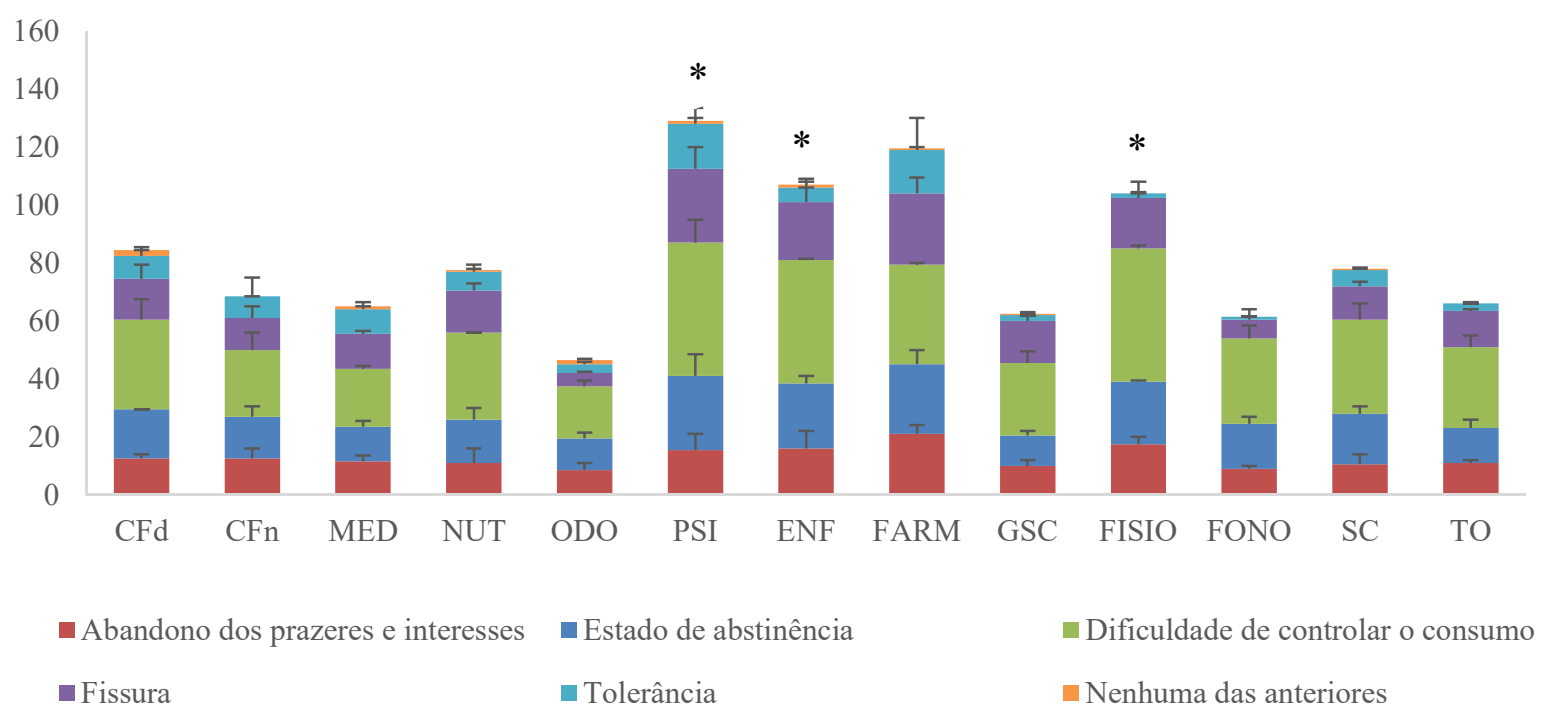

Figura 19: Características da dependência química. As colunas representam as médias e as barras o EPM. $\mathrm{p}<0,05$ de acordo com a ANOVA One-Way. * Indica diferença significativa de acordo com o teste de Student-Newman-Keuls (vide texto) $(\mathrm{n}=944)$.

\begin{tabular}{l}
\hline 12. Como você acha que poderia se dar o aprendizado sobre uso \\
de drogas e dependência química durante a graduação? (Assinale \\
todas as alternativas que se aplicam) \\
\hline Aulas expositivas \\
\hline Congressos \\
\hline Cursos de extensão
\end{tabular}




\begin{tabular}{l}
\hline Palestras \\
\hline Projetos de iniciação científica \\
\hline Outros
\end{tabular}

Na Questão 12, a ANOVA One-Way, seguida pelo teste de Student-Newman-Keuls, revelou que houve diferença significativa nas alternativas Congressos entre os cursos Psicologia $(\bar{X}=25.5 ; \mathrm{EPM}=3.50)$ e Medicina $(\bar{X}=8.50 ; \mathrm{EPM}=0.50)$, Psicologia $(\bar{X}=$ $25.5 ; \mathrm{EPM}=3.50)$ e Odontologia $(\bar{X}=6.50 ; \mathrm{EPM}=1.50)$, Farmácia $(\bar{X}=24 ; \mathrm{EPM}=3)$ e Medicina $(\bar{X}=8.50 ; \mathrm{EPM}=0.50)$, Farmácia $(\bar{X}=24 ; \mathrm{EPM}=3)$ e Odontologia $(\bar{X}=6.50$; $\mathrm{EPM}=1.50)$, Enfermagem $(\bar{X}=22.5 ; \mathrm{EPM}=3.50)$ e Odontologia $(\bar{X}=6.50 ; \mathrm{EPM}=0.50)$, com $\mathrm{F}_{(12,931)}=4.25$ e $\mathrm{p}=0.007$; Cursos de extensão entre os cursos Psicologia $(\bar{X}=34$; EPM $=5)$ e Medicina $(\bar{X}=13 ; \mathrm{EPM}=0)$, Psicologia $(\bar{X}=34 ; \mathrm{EPM}=5)$ e Odontologia $(\bar{X}=$ 5.50; $\mathrm{EPM}=2.50)$, Farmácia $(\bar{X}=27.5 ; \mathrm{EPM}=6.50)$ e Medicina $(\bar{X}=13 ; \mathrm{EPM}=0)$, Farmácia $(\bar{X}=27.5 ; \mathrm{EPM}=6.50)$ e Odontologia $(\bar{X}=5.50 ; \mathrm{EPM}=2.50)$, com $\mathrm{F}_{(12,931)}=$ 3.53 e $\mathrm{p}=0.01$; Palestras entre os cursos Fisioterapia $(\bar{X}=41 ; \mathrm{EPM}=5)$ e Medicina $(\bar{X}=$ 13.5; $\mathrm{EPM}=1.50)$, com $\mathrm{F}_{(12,931)}=2.76 \mathrm{e} \mathrm{p}=0.04$, Projetos de iniciação científica entre os cursos Psicologia $(\bar{X}=31 ; \mathrm{EPM}=6)$ e Medicina $(\bar{X}=13 ; \mathrm{EPM}=1)$, Psicologia $(\bar{X}=31$; $\mathrm{EPM}=6)$ e Odontologia $(\bar{X}=9.50 ; \mathrm{EPM}=1.50)$, Fisioterapia $(\bar{X}=26.5 ; \mathrm{EPM}=2.50) \mathrm{e}$ Odontologia $(\bar{X}=9.50 ; \mathrm{EPM}=1.50)$, Farmácia $(\bar{X}=27 ; \mathrm{EPM}=4)$ e Odontologia $(\bar{X}=$ 9.50; $\mathrm{EPM}=1.50)$, Enfermagem $(\bar{X}=27 ; \mathrm{EPM}=1)$ e Odontologia $(\bar{X}=9.50 ; \mathrm{EPM}=1.50)$, $\operatorname{com} \mathrm{F}_{(12,931)}=4.80$ e $\mathrm{p}=0.004$ e Outros entre os cursos Psicologia $(\bar{X}=14 ; \mathrm{EPM}=3)$ e Ciências Farmacêuticas diurno $(\bar{X}=3.50 ; \mathrm{EPM}=2.50)$, Psicologia $(\bar{X}=14 ; \mathrm{EPM}=3) \mathrm{e}$ Ciências Farmacêuticas noturno $(\bar{X}=4.50 ; \mathrm{EPM}=2.50)$, Psicologia $(\bar{X}=14 ; \mathrm{EPM}=3) \mathrm{e}$ Medicina $(\bar{X}=3.50 ; \mathrm{EPM}=0.50)$, Psicologia $(\bar{X}=14 ; \mathrm{EPM}=3)$ e Nutrição $(\bar{X}=4 ; \mathrm{EPM}=$ 1), Psicologia $(\bar{X}=14 ; \mathrm{EPM}=3)$ e Odontologia $(\bar{X}=4.50 ; \mathrm{EPM}=1.50)$, Psicologia $(\bar{X}=$ 14; $\mathrm{EPM}=3)$ e Enfermagem $(\bar{X}=6 ; \mathrm{EPM}=2)$, Psicologia $(\bar{X}=14 ; \mathrm{EPM}=3)$ e Gestão em Saúde Coletiva $(\bar{X}=7.50 ; \mathrm{EPM}=0,40)$, Psicologia $(\bar{X}=5,67 ; \mathrm{EPM}=0,23) \mathrm{e}$ Fonoaudiologia $(\bar{X}=4,36 ; \mathrm{EPM}=1.50)$, Psicologia $(\bar{X}=14 ; \mathrm{EPM}=3)$ e Saúde Coletiva ( 
$\bar{X}=3 ; \mathrm{EPM}=0)$, Psicologia $(\bar{X}=14 ; \mathrm{EPM}=3)$ e Terapia Ocupacional $(\bar{X}=4.50 ; \mathrm{EPM}=$ $1.50)$, com $\mathrm{F}_{(12,931)}=3.37$ e $\mathrm{p}=0,01$. Não houve diferença significativa na alternativa Aulas expositivas $\mathrm{F}_{(12,931)}=2.02$ e $\mathrm{p}=0.11$ (Figura 20).

\section{Formas de aprendizado sobre o uso de drogas e dependência química}

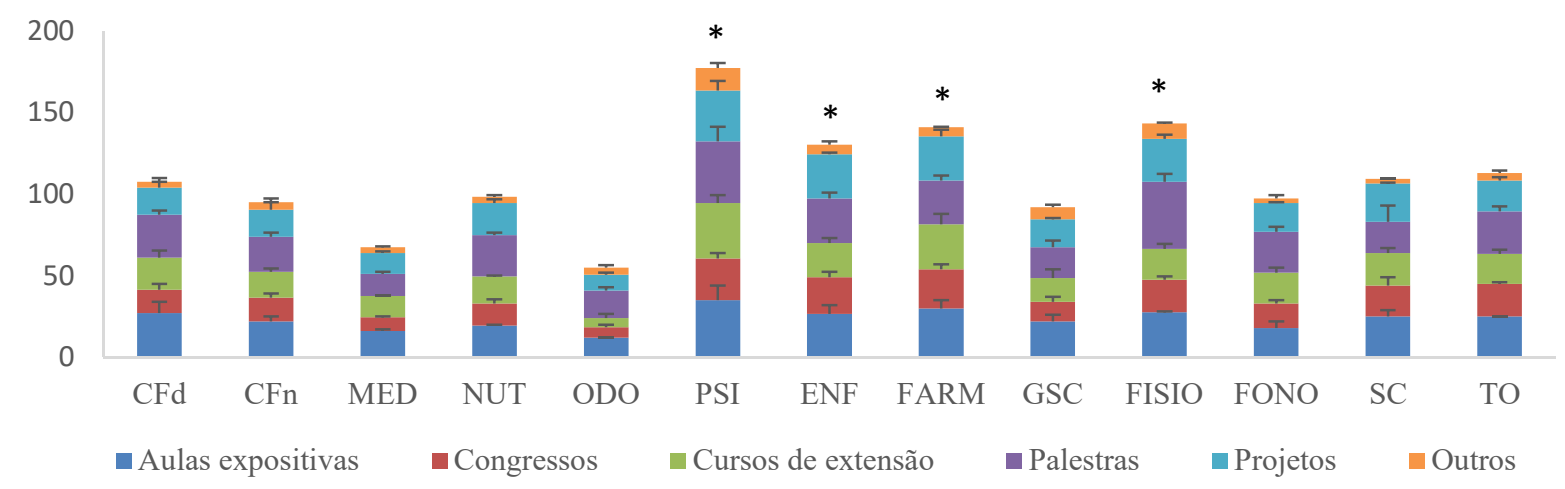

Figura 20: Formas de aprendizado sobre o uso de drogas e dependência química. As colunas representam as médias e as barras o EPM. $\mathrm{p}<0,05$, de acordo com a ANOVA One-Way. * Indica diferença significativa de acordo com o teste de Student-Newman- Keuls (vide texto) $(n=944)$.

\begin{tabular}{l}
\hline 16. Você acha que a formação sobre uso de drogas e dependência \\
química é relevante no seu curso? \\
\hline Sim \\
\hline Não
\end{tabular}

Na Questão 16, a ANOVA One-Way, seguida pelo teste de Student-Newman- Keuls, revelou que houve diferença significativa na alternativa Sim entre os cursos Enfermagem ( $\bar{X}$ $=6 ; \mathrm{EPM}=2)$ e Odontologia $(\bar{X}=22.5 ; \mathrm{EPM}=2.50)$, $\operatorname{com} \mathrm{F}_{(12,931)}=4.14$ e $\mathrm{p}=0.008$. Não

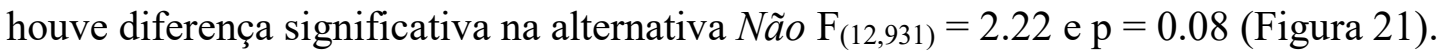

\section{Opinião sobre a relevância da formação a respeito da dependência química}

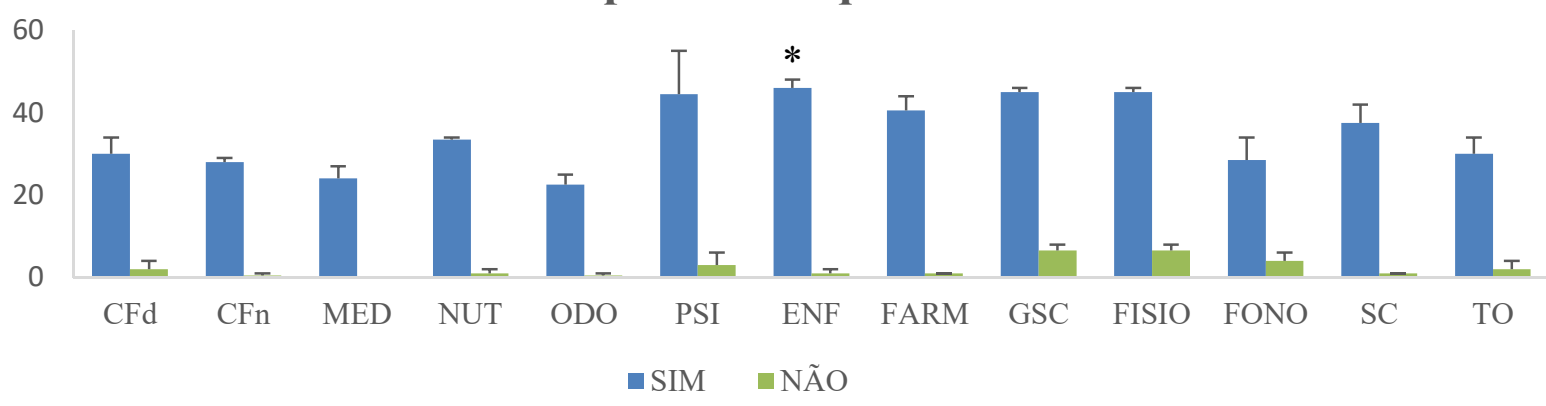

Figura 21: Opinião sobre a relevância da formação sobre dependência química. As colunas representam as médias e as barras o EPM. $\mathrm{p}<0,05$ de acordo com a ANOVA One-Way. * 
Indica diferença significativa entre Enfermagem e Odontologia, de acordo com o teste de Student-Newman-Keuls $(\mathrm{n}=944)$.

\begin{tabular}{l}
\hline $\begin{array}{l}\text { 17. Você já usou alguma(s) da(s) substância(s) a seguir? (Assinale } \\
\text { todas as alternativas que se aplicam) }\end{array}$ \\
\hline Álcool \\
\hline Alucinógeno (LSD, chá de ayahuasca) \\
\hline Anfetamina (metanfetamina, metilfenidato) \\
\hline Benzodiazepínico ou barbitúrico (clonazepam, fenobarbital) \\
\hline Cigarro de nicotina \\
\hline Cocaína ou merla ou crack \\
\hline Esteroide anabolizante (testosterona) \\
\hline Inalantes (loló, lança-perfume) \\
\hline Maconha ou haxixe ou skank \\
\hline Opiáceo (codeína, morfina, meperidina)/ Opióide (heroína) \\
\hline
\end{tabular}

Na Questão 17, a ANOVA One-Way, seguida pelo teste de Student-Newman- Keuls, revelou que houve diferença significativa nas alternativas Álcool entre os cursos Psicologia ( $\bar{X}=43 ; \mathrm{EPM}=6)$ e Medicina $(\bar{X}=22 ; \mathrm{EPM}=5)$, Psicologia $(\bar{X}=43 ; \mathrm{EPM}=6)$ e

Odontologia $(\bar{X}=21 ; \mathrm{EPM}=3)$, Fisioterapia $(\bar{X}=41.5 ; \mathrm{EPM}=2.50)$ e Medicina $(\bar{X}=22$; $\mathrm{EPM}=5)$, Fisioterapia $(\bar{X}=41.5 ; \mathrm{EPM}=2.50)$ e Odontologia $(\bar{X}=21 ; \mathrm{EPM}=3)$, Enfermagem $(\bar{X}=40.5 ; \mathrm{EPM}=1.50)$ e Odontologia $(\bar{X}=21 ; \mathrm{EPM}=3), \operatorname{com~}_{(12,931)}=4.86$ e $\mathrm{p}=0.004 ;$ Anfetaminas entre os cursos Farmácia $(\bar{X}=4 ; \mathrm{EPM}=1)$ e Fisioterapia $(\bar{X}=0$; $\mathrm{EPM}=0)$, com $\mathrm{F}_{(12,931)}=3.41$ e $\mathrm{p}=0.01$; Cigarro de nicotina entre os cursos Farmácia $(\bar{X}=$ 16.5; $\mathrm{EPM}=0.50)$ e Odontologia $(\bar{X}=5 ; \mathrm{EPM}=2), \operatorname{com~}_{(12,931)}=2.52$ e $\mathrm{p}=0.05$ e Esteroides anabolizantes entre os cursos Nutrição $(\bar{X}=2.50 ; \mathrm{EPM}=0.50)$ e Ciências Farmacêuticas diurno $(\bar{X}=0 ; \mathrm{EPM}=0)$, Nutrição $(\bar{X}=2.50 ; \mathrm{EPM}=0.50)$ e Ciências Farmacêuticas noturno $(\bar{X}=0.50 ; \mathrm{EPM}=0.50)$, Nutrição $(\bar{X}=2.50 ; \mathrm{EPM}=0.50) \mathrm{e}$ Medicina $(\bar{X}=0 ; \mathrm{EPM}=0)$, Nutrição $(\bar{X}=2.50 ; \mathrm{EPM}=0.50)$ e Odontologia $(\bar{X}=0.50$; $\mathrm{EPM}=0.50)$, Nutrição $(\bar{X}=2.50 ; \mathrm{EPM}=0.50)$ e Psicologia $(\bar{X}=0 ; \mathrm{EPM}=0)$, Nutrição $(\bar{X}$ $=2.50 ; \mathrm{EPM}=0.50)$ e Enfermagem $(\bar{X}=0 ; \mathrm{EPM}=0)$, Nutrição $(\bar{X}=2.50 ; \mathrm{EPM}=0.50)$ e Fonoaudiologia $(\bar{X}=0 ; \mathrm{EPM}=0)$, Nutrição $(\bar{X}=2.50 ; \mathrm{EPM}=0.50)$ e Saúde Coletiva $(\bar{X}=$ $0.50 ; \mathrm{EPM}=0.50)$, Nutrição $(\bar{X}=2.50 ; \mathrm{EPM}=0.50)$ e Terapia Ocupacional $(\bar{X}=0 ; \mathrm{EPM}=$ 0 ), com $\mathrm{F}_{(12,931)}=5.29$ e $\mathrm{p}=0.002$ (Figura 46). Não houve diferença significativa nas 
alternativas: Alucinógenos com $\mathrm{F}_{(12,931)}=0.82$ e $\mathrm{p}=0.62$; Benzodiazepínicos e barbitúricos com $\mathrm{F}_{(12,931)}=0.46$ e $\mathrm{p}=0.90 ;$ Cocaína ou merla ou crack $\operatorname{com} \mathrm{F}_{(12,931)}=0.42$ e $\mathrm{p}=0.92$;

Inalantes com $\mathrm{F}_{(12,931)}=0.43$ e $\mathrm{p}=0.90 ;$ Maconha ou haxixe ou skank com $\mathrm{F}_{(12,931)}=1.15$ e $\mathrm{p}$ $=0.40$ e Opiáceo/Opióide com $\mathrm{F}_{(12,931)}=1.63$ e $\mathrm{p}=0.19$ (Figura 22).

\section{Uso de drogas psicotrópicas por estudantes}

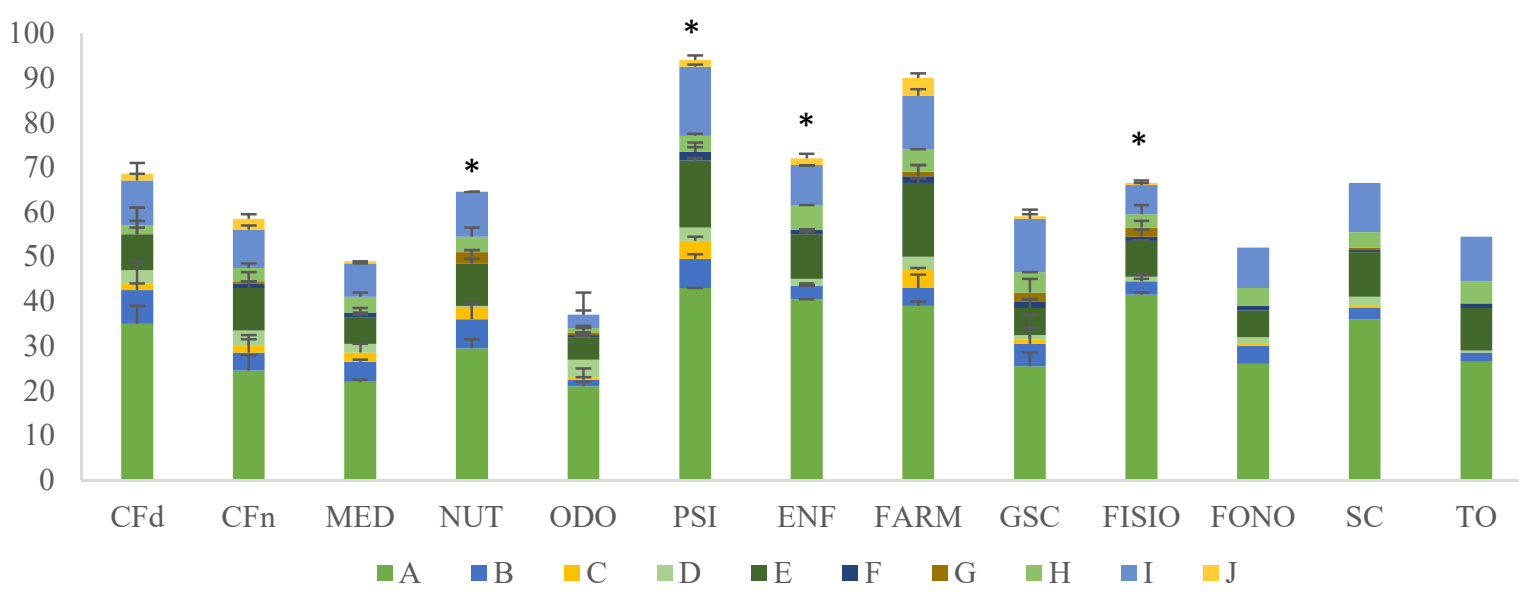

Figura 22: Consumo drogas psicotrópicas entre estudantes de todos os cursos. As colunas representam as médias das alternativas A - Álcool, B - Alucinógeno, C - Anfetamina, D Benzodiazepínico ou barbitúrico, E - Cigarro de nicotina, F - Cocaína ou merla ou crack, G Esteroide anabolizante, H - Inalantes, I - Maconha ou haxixe ou skank e J - Opiáceo/Opióide e as barras o EPM. $\mathrm{p}<0,05$, de acordo com a ANOVA One-Way. * Indica diferença significativa de acordo com o teste de Student-Newman- Keuls (vide texto) ( $\mathrm{n}=944)$.

\begin{tabular}{l}
18 . Você usou alguma das substâncias da questão 17 nos últimos \\
30 dias? \\
\hline Sim \\
\hline Não \\
\hline Não se aplica
\end{tabular}

\begin{tabular}{l}
$\begin{array}{l}\text { 19. Você usou alguma das substâncias da questão } 17 \text { no último } \\
\text { ano? }\end{array}$ \\
\hline Sim \\
\hline Não \\
\hline Não se aplica
\end{tabular}

Nas questões 18 e 19, a ANOVA One-Way, seguida pelo teste de Student-NewmanKeuls, revelou que apenas na alternativa Sim no último ano houve diferença significativa entre os cursos Psicologia $(\bar{X}=38 ; \mathrm{EPM}=7)$ e Odontologia $(\bar{X}=18 ; \mathrm{EPM}=2)$, com $\mathrm{F}_{(12,931)}=2.83$ e $\mathrm{p}=0.03$. Não houve diferença significativa na opção Sim nos últimos 30 dias 
com $F_{(12,931)}=2.20$ e $p=0.08$ (Figura 23). Essas análises foram feitas separadamente, mas optou-se por apresentar os dados conjuntamente.

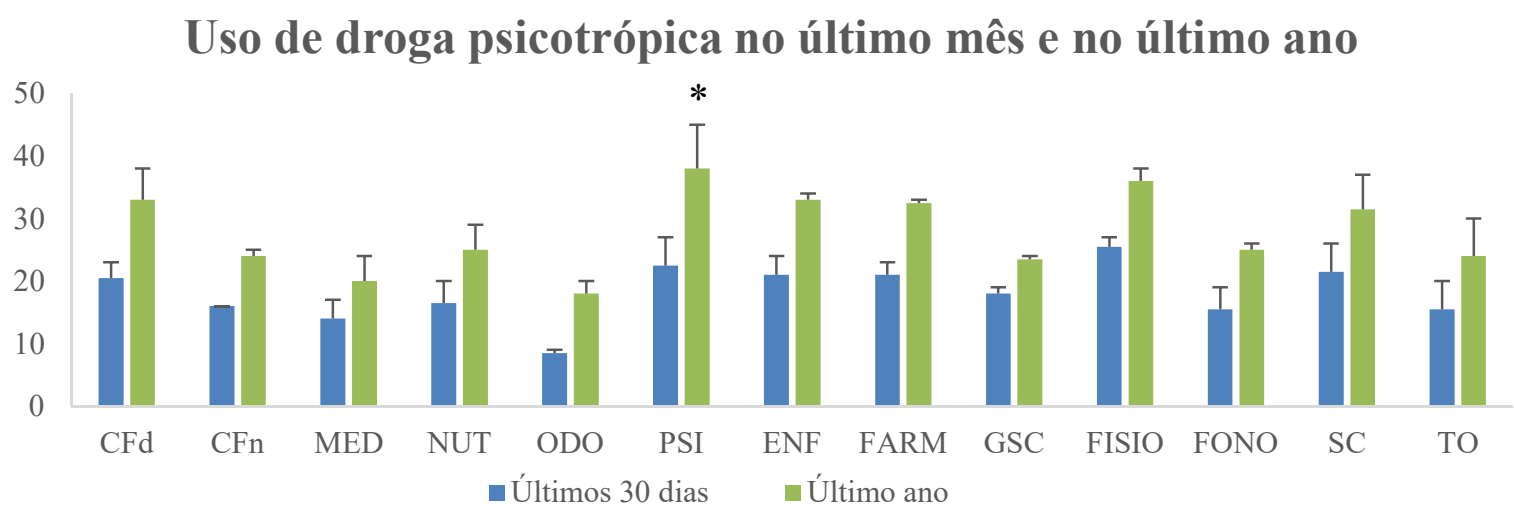

Figura 23: Uso de droga psicotrópica nos últimos 30 dias e no último ano. As colunas representam as médias e as barras o EPM. $\mathrm{p}<0,05$, de acordo com a ANOVA One-Way. * Indica diferença significativa entre Psicologia e Odontologia, de acordo com o teste de Student-Newman-Keuls $(\mathrm{n}=944)$.

\begin{tabular}{l}
\hline $\begin{array}{l}\text { 20. Que motivo o levou a fazer uso de alguma das substâncias citadas } \\
\text { anteriormente pela primeira vez? (Assinale todas as alternativas que se } \\
\text { aplicam) }\end{array}$ \\
\hline Alívio de cansaço \\
\hline Alívio de dor \\
\hline Aumentar desempenho de estudo \\
\hline Curiosidade \\
\hline Diversão/prazer \\
\hline Manter-se acordado(a) \\
\hline Melhorar desempenho sexual \\
\hline Perder inibição \\
\hline Porque amigos/namorado(a) usam \\
\hline Prática religiosa \\
\hline Relaxar \\
\hline Outro
\end{tabular}

Na Questão 20, a respeito do motivo para o uso de drogas psicotrópicas entre os estudantes, a ANOVA One-Way, seguida pelo teste de Student-Newman- Keuls, revelou que houve diferença significativa nas alternativas Perder inibição entre os cursos Psicologia ( $\bar{X}$ $=14 ; \mathrm{EPM}=2)$ e Ciências Farmacêuticas diurno $(\bar{X}=4.50 ; \mathrm{EPM}=1.50)$, Psicologia $(\bar{X}=$ 14; $\mathrm{EPM}=2)$ e Ciências Farmacêuticas noturno $(\bar{X}=5 ; \mathrm{EPM}=2)$, Psicologia $(\bar{X}=14 ; \mathrm{EPM}$ $=2)$ e Medicina $(\bar{X}=6 ; \mathrm{EPM}=3)$, Psicologia $(\bar{X}=14 ; \mathrm{EPM}=2)$ e Nutrição $(\bar{X}=8 ; \mathrm{EPM}=$ 1), Psicologia $(\bar{X}=14 ; \mathrm{EPM}=2)$ e Odontologia $(\bar{X}=2 ; \mathrm{EPM}=1)$, Psicologia $(\bar{X}=14$; 
$\mathrm{EPM}=2)$ e Enfermagem $(\bar{X}=3.50 ; \mathrm{EPM}=1.50)$, Psicologia $(\bar{X}=14 ; \mathrm{EPM}=2)$ e Farmácia $(\bar{X}=5.50 ; \mathrm{EPM}=2.50)$, Psicologia $(\bar{X}=14 ; \mathrm{EPM}=2)$ e Gestão em Saúde Coletiva $(\bar{X}=$ 3.50; $\mathrm{EPM}=1.50)$, Psicologia $(\bar{X}=14 ; \mathrm{EPM}=2)$ e Fisioterapia $(\bar{X}=7 ; \mathrm{EPM}=2)$, Psicologia $(\bar{X}=14 ; \mathrm{EPM}=2)$ e Fonoaudiologia $(\bar{X}=2.50 ; \mathrm{EPM}=0.50)$, Psicologia $(\bar{X}=$ 14; $\mathrm{EPM}=2)$ e Saúde Coletiva $(\bar{X}=4 ; \mathrm{EPM}=0)$ e Psicologia $(\bar{X}=14 ; \mathrm{EPM}=2)$ e Terapia Ocupacional $(\bar{X}=3 ; \mathrm{EPM}=1)$, com $\mathrm{F}_{(12,931)}=3.47$ e $\mathrm{p}=0.01 ;$ Porque amigos $/$ namorado usam os cursos Psicologia $(\bar{X}=12 ; \mathrm{EPM}=0)$ e Ciências Farmacêuticas diurno $(\bar{X}=4$; EPM =1), Psicologia $(\bar{X}=12 ; \mathrm{EPM}=0)$ e Ciências Farmacêuticas noturno $(\bar{X}=4 ; \mathrm{EPM}=1)$, Psicologia $(\bar{X}=12 ; \mathrm{EPM}=0)$ e Medicina $(\bar{X}=3 ; \mathrm{EPM}=1)$, Psicologia $(\bar{X}=12 ; \mathrm{EPM}=0)$ e Gestão em Saúde Coletiva $(\bar{X}=4.50 ; \mathrm{EPM}=0.50)$, Psicologia $(\bar{X}=12 ; \mathrm{EPM}=0)$ e Fonoaudiologia $(\bar{X}=3.50 ; \mathrm{EPM}=0.50)$ e Psicologia $(\bar{X}=12 ; \mathrm{EPM}=0)$ e Terapia Ocupacional $(\bar{X}=4.50 ; \mathrm{EPM}=0.50), \operatorname{com} \mathrm{F}_{(12,931)}=2.87$ e $\mathrm{p}=0.03$ e Prática Religiosa entre os cursos Psicologia $(\bar{X}=2 ; \mathrm{EPM}=1)$ e Enfermagem $(\bar{X}=0 ; \mathrm{EPM}=0), \operatorname{com} \mathrm{F}_{(12,931)}=3.23$ e $\mathrm{p}=0.02$. Não houve diferença significativa nas alternativas Alívio de cansaço $\mathrm{F}_{(12,931)}=1.62$ e $\mathrm{p}=0.20 ;$ Alívio de dor $\mathrm{F}_{(12,931)}=1.30$ e $\mathrm{p}=0.32 ;$ Aumentar desempenho de estudo $\mathrm{F}_{(12,931)}=$ 1.61 e $\mathrm{p}=0.20 ;$ Curiosidade $\mathrm{F}_{(12,931)}=1.82$ e $\mathrm{p}=0.14 ;$ Diversão/Prazer $\mathrm{F}_{(12,931)}=2.17$ e $\mathrm{p}=$ 0.09; Manter-se acordado(a) $\mathrm{F}_{(12,931)}=1.88$ e $\mathrm{p}=0.13 ;$ Melhorar desempenho sexual $\mathrm{F}_{(12,931)}$ $=0.75$ e $\mathrm{p}=0.68 ;$ Relaxar $\mathrm{F}_{(12,931)}=2.36$ e $\mathrm{p}=0.06$ Outro $\mathrm{F}_{(12,931)}=1.82$ e $\mathrm{p}=0.14$ (Figura 24$)$. 


\section{Motivos para o uso de drogas psicotrópicas entre estudantes}

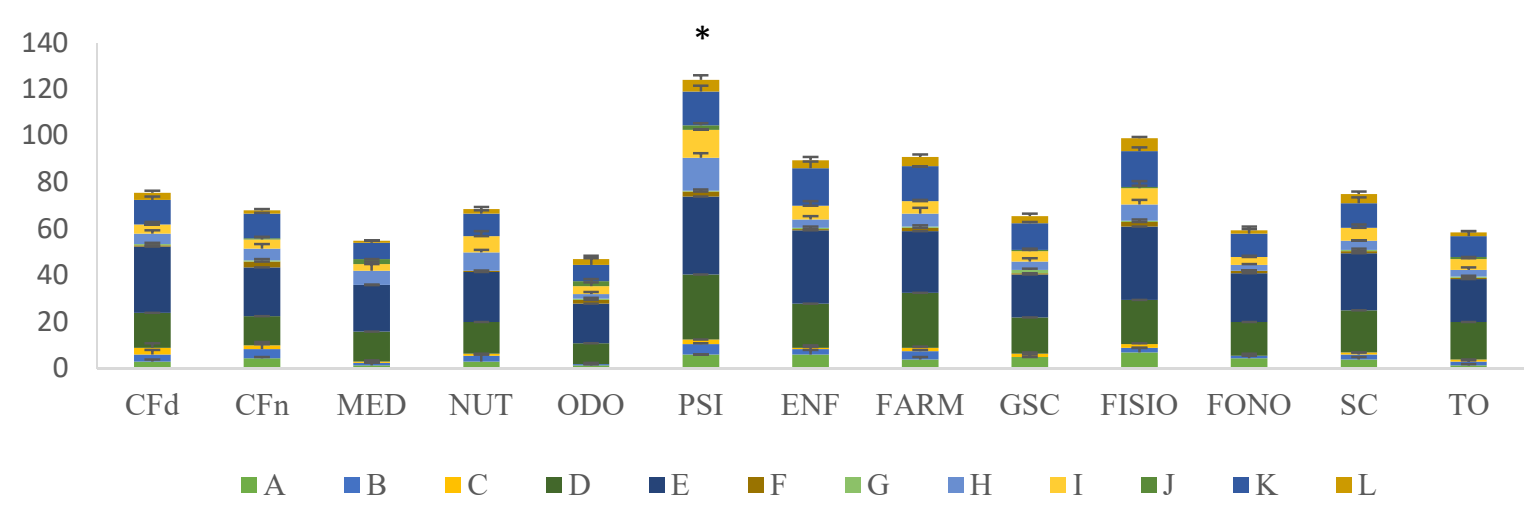

Figura 24: Motivos para o uso de drogas psicotrópicas entre estudantes. As colunas representam as médias das alternativas: A - Alívio de cansaço, B - Alívio de dor, C Aumentar desempenho de estudo, D - Curiosidade, E - Diversão/prazer, F - Manter-se acordado(a), G - Melhorar desempenho sexual, H - Perder inibição, I - Porque amigos/namorado(a) usam, J - Prática religiosa, K - Relaxar, L - Outro e as barras o EPM. p < 0,05 , de acordo com a ANOVA One-Way * Indica diferença significativa de acordo com o teste de Student-Newman-Keuls (vide texto) $(\mathrm{n}=944)$.

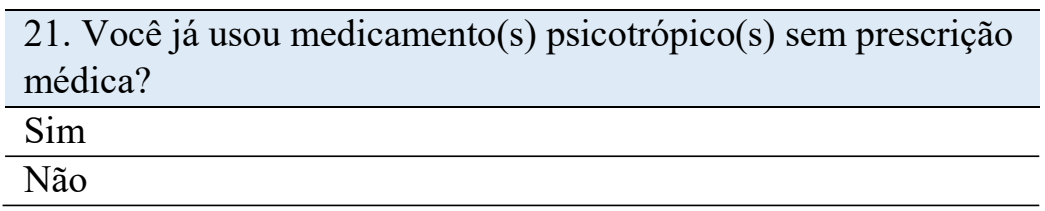

Na Questão 21, a ANOVA One-Way, seguida pelo teste de Student-Newman- Keuls, revelou que houve diferença significativa na alternativa $N a \tilde{o}$ entre os cursos Fisioterapia $(\bar{X}=$ 46; $\mathrm{EPM}=0)$ e Odontologia $(\bar{X}=2.50 ; \mathrm{EPM}=1.50), \operatorname{com}_{(12,931)}=3.26$ e $\mathrm{p}=0.02 . \mathrm{Na}$ alternativa Sim não houve diferença significativa $\mathrm{F}_{(12,931)}=0.64$ e $\mathrm{p}=0.77$ (Figura 25).

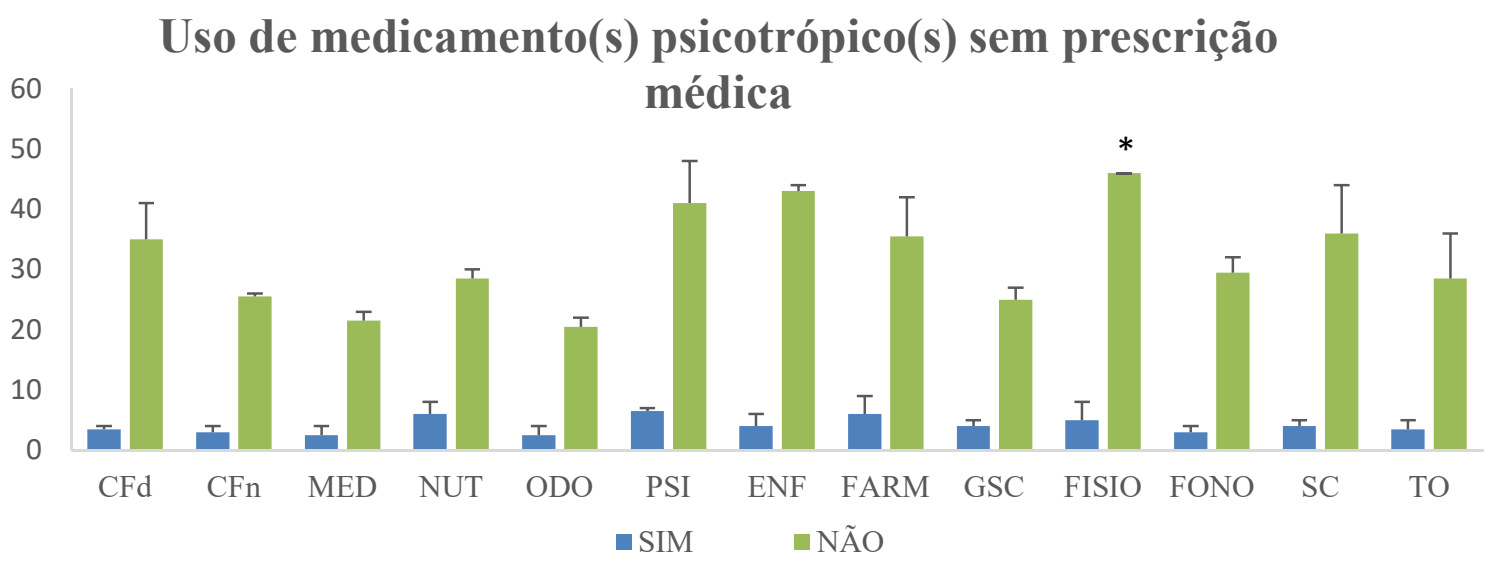

Figura 25: Uso de medicamentos psicotrópicos entre estudantes sem prescrição médica. As colunas representam as médias e as barras o EPM. $\mathrm{p}<0,05$, de acordo com a ANOVA OneWay. * Indica diferença significativa entre Fisioterapia e Odontologia, de acordo com o teste de Student-Newman-Keuls ( $\mathrm{n}=944)$. 


\begin{tabular}{l}
\hline $\begin{array}{l}\text { 22. Se você julgar que existe tratamento para dependência química, } \\
\text { como deveria ser? (Assinale todas as alternativas que se aplicam) }\end{array}$ \\
\hline Eletrochoque \\
\hline Internação em clínica de orientação religiosa \\
\hline Internação voluntária \\
\hline Internação compulsória \\
\hline Psicoterapia \\
\hline Uso de medicamentos \\
\hline Não há tratamento \\
\hline Nenhum dos tratamentos anteriores
\end{tabular}

Na Questão 22, a ANOVA One-Way, seguida pelo teste de Student-Newman- Keuls, revelou que houve diferença significativa nas alternativas Internação voluntária entre os cursos Fisioterapia $(\bar{X}=45.5 ; \mathrm{EPM}=1.50)$ e Odontologia $(\bar{X}=17.50 ; \mathrm{EPM}=1.50) \mathrm{e}$ Fisioterapia $(\bar{X}=45.5 ; \mathrm{EPM}=1.50)$ e Medicina $(\bar{X}=19 ; \mathrm{EPM}=1), \operatorname{com}_{(12,931)}=3.71$ e $\mathrm{p}$ $=0.01 ;$ Internação compulsória entre os cursos Enfermagem $(\bar{X}=17 ; \mathrm{EPM}=0)$ e Terapia Ocupacional $(\bar{X}=3.50 ; \mathrm{EPM}=1.50), \operatorname{com~}_{(12,931)}=3.08$ e $\mathrm{p}=0.02$ e Psicoterapia entre os cursos Psicologia $(\bar{X}=45.5 ; \mathrm{EPM}=6.50)$ e Ciências Farmacêuticas noturno $(\bar{X}=22.5$; $\mathrm{EPM}=5.50)$, Psicologia $(\bar{X}=45.5 ; \mathrm{EPM}=6.50)$ e Medicina $(\bar{X}=21.5 ; \mathrm{EPM}=1.50)$, Psicologia $(\bar{X}=45.5 ; \mathrm{EPM}=6.50)$ e Odontologia $(\bar{X}=15.5 ; \mathrm{EPM}=0.50)$, Psicologia $(\bar{X}=$ $45.5 ; \mathrm{EPM}=6.50)$ e Gestão em Saúde Coletiva $(\bar{X}=22 ; \mathrm{EPM}=4)$, Psicologia $(\bar{X}=45.5$; $\mathrm{EPM}=6.50)$ e Fonoaudiologia $(\bar{X}=23 ; \mathrm{EPM}=7)$, Psicologia $(\bar{X}=45.5 ; \mathrm{EPM}=6.50) \mathrm{e}$ Terapia Ocupacional $(\bar{X}=23.5 ; \mathrm{EPM}=1.50), \operatorname{com} \mathrm{F}_{(12,931)}=3.71$ e $\mathrm{p}=0.01$. Não houve diferença significativa nas alternativas Eletrochoque $\mathrm{F}_{(12,931)}=1.12$ e $\mathrm{p}=0.41$; Internação em clínica religiosa $\mathrm{F}_{(12,931)}=1.72$ e $\mathrm{p}=0.17$; Uso de medicamentos $\mathrm{F}_{(12,931)}=1.29$ e $\mathrm{p}=0.32$; Não há tratamento $\mathrm{F}_{(12,931)}=0.91$ e $\mathrm{p}=0.55$ e Nenhum dos anteriores $\mathrm{F}_{(12,931)}=1.38$ e $\mathrm{p}=$ 0.28 (Figura 26). 


\section{Conhecimento sobre tratamentos para dependência química}

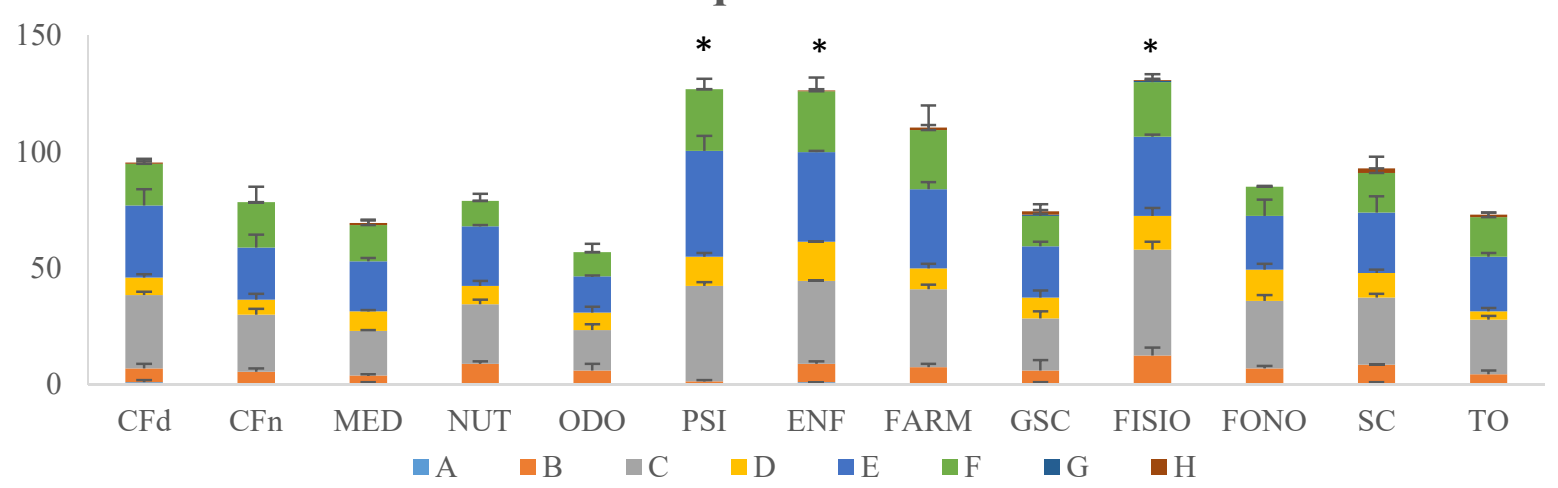

Figura 26: Conhecimento sobre diferentes modalidades de tratamento para a dependência química. As colunas representam as médias das alternativas A - Eletrochoque, B - Internação em clínica de orientação religiosa, C - Internação voluntária, D - Internação Compulsória, EPsicoterapia, F - Uso de medicamentos, G - Não há tratamento, H - Nenhum dos anteriores e as barras o EPM. $\mathrm{p}<0,05$, de acordo com a ANOVA One-Way. * Indica diferença significativa de acordo com o teste de Student-Newman-Keuls (vide texto) $(\mathrm{n}=944)$.

A seguir são apresentadas as ANOVA One-Way, seguidas pelo teste de StudentNewman-Keuls $(\mathrm{p}<0,05)$, das combinações com maior frequência de resposta, obtidas a partir das questões em que havia a possibilidade de assinalar mais de uma alternativa.

Na Questão 10, a análise da combinação entre as alternativas Tem cura e Tem tratamento revelou que houve diferença significativa apenas entre os cursos Psicologia $(\bar{X}=$ $11 ; \mathrm{EPM}=1)$ e Terapia Ocupacional $(\bar{X}=0.50 ; \mathrm{EPM}=0 ; 50), \operatorname{com} \mathrm{F}_{(12,146)}=2.49$ e $\mathrm{p}=0.05$ (Figura 27).

\section{Combinação respostas Questão 10}

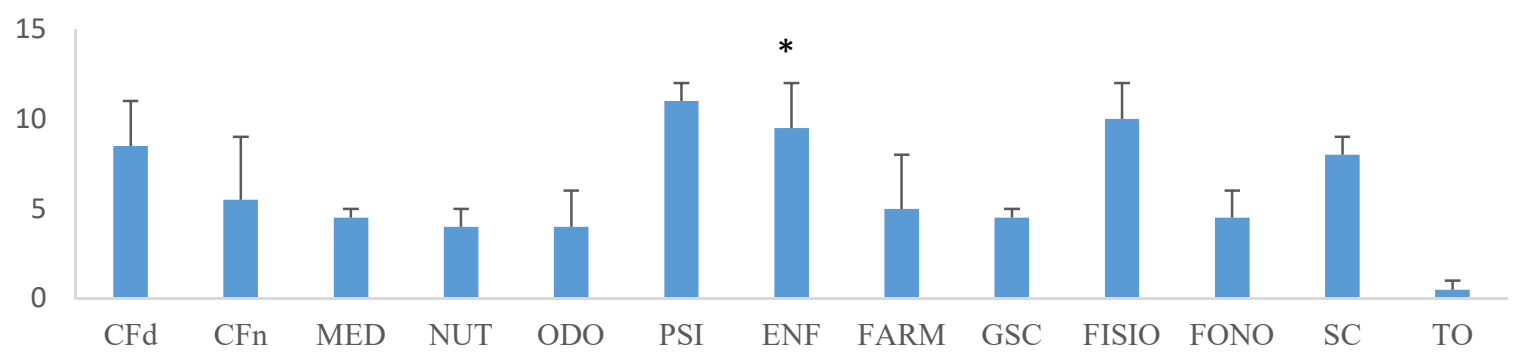

Figura 27: Combinação mais frequente entre as alternativas Tem cura e Tem tratamento da Questão 10. As colunas representam as médias e as barras o EPM. $\mathrm{p}<0,05$, de acordo com a ANOVA One-Way. * Indica diferença significativa entre Psicologia e Terapia Ocupacional, de acordo com o teste de Student-Newman-Keuls ( $\mathrm{n}$ total $=159)$. 
Na Questão 11, a análise da combinação, por meio da ANOVA One-Way, seguida pelo teste de Student-Newman-Keuls, revelou que entre as alternativas Dificuldade de controlar o consumo e Persistência no uso houve diferença significativa entre os cursos Fisioterapia $(\bar{X}=8.50 ; \mathrm{EPM}=1.50)$ e Ciências Farmacêuticas diurno $(\bar{X}=1.50 ; \mathrm{EPM}=$ $1 ; 50)$, Fisioterapia $(\bar{X}=8.50 ; \mathrm{EPM}=1.50)$ e Medicina $(\bar{X}=1 ; \mathrm{EPM}=0)$, Fisioterapia $(\bar{X}=$ 8.50; $\mathrm{EPM}=1.50)$ e Odontologia $(\bar{X}=1 ; \mathrm{EPM}=1)$, Fisioterapia $(\bar{X}=8.50 ; \mathrm{EPM}=1.50) \mathrm{e}$ Farmácia $(\bar{X}=1 ; E P M=1)$, Fonoaudiologia $(\bar{X}=8.50 ; E P M=0.50)$ e Ciências Farmacêuticas diurno $(\bar{X}=1.50 ; \mathrm{EPM}=1 ; 50)$, Fonoaudiologia $(\bar{X}=8.50 ; \mathrm{EPM}=0.50) \mathrm{e}$ Medicina $(\bar{X}=1 ; \mathrm{EPM}=0)$, Fonoaudiologia $(\bar{X}=8.50 ; \mathrm{EPM}=0.50)$ e Odontologia $(\bar{X}=1$; $\mathrm{EPM}=1)$, Fonoaudiologia $(\bar{X}=8.50 ; \mathrm{EPM}=0.50)$ e Farmácia $(\bar{X}=1 ; \mathrm{EPM}=1)$, com $\mathrm{F}_{(1,943)}=4.15$ e $\mathrm{p}=0$ (Figura 28).

\section{Combinação respostas Questão 11}

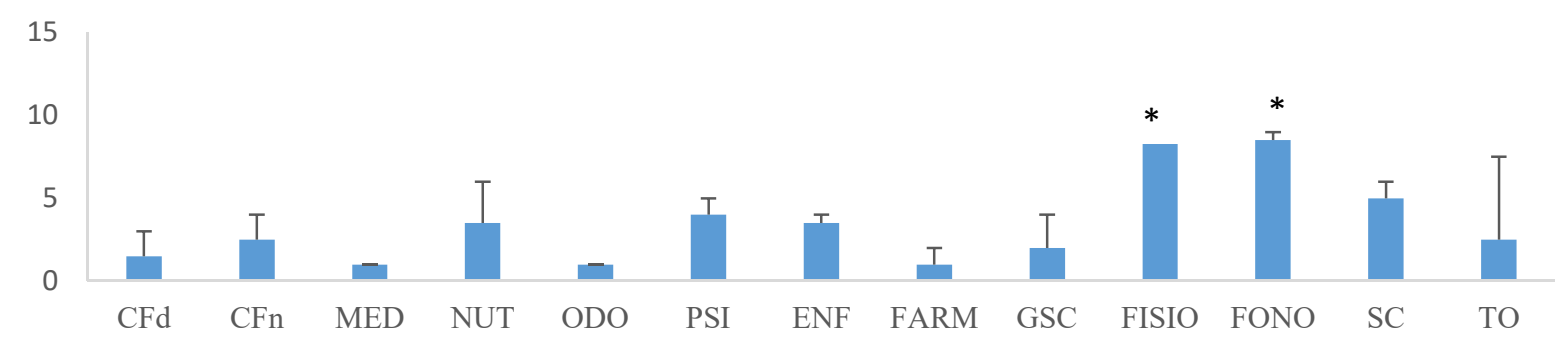

Figura 28: As colunas representam as médias e as barras o EPM da combinação das respostas Dificuldade de controlar o consumo e Persistência no uso da Questão 12. $\mathrm{p}<0,05$ de acordo com ANOVA One-Way. * Indica diferença significativa de acordo com o teste de StudentNewman-Keuls $(\mathrm{n}$ total $=76)$.

Na Questão 20, a análise da combinação, por meio da ANOVA One-Way, seguida pelo teste de Student-Newman-Keuls, entre as alternativas Alívio de cansaço e Aumentar desempenho de estudo revelou que houve diferença significativa entre os cursos Fisioterapia ( $\bar{X}=9 ; \mathrm{EPM}=1)$ e Ciências Farmacêuticas diurno $(\bar{X}=2.50 ; \mathrm{EPM}=1 ; 50)$, Fisioterapia $(\bar{X}$ $=9 ; \mathrm{EPM}=1)$ e Medicina $(\bar{X}=1.50 ; \mathrm{EPM}=1.50)$, Fisioterapia $(\bar{X}=9 ; \mathrm{EPM}=1) \mathrm{e}$ Odontologia $(\bar{X}=2 ; \mathrm{EPM}=0)$, Fisioterapia $(\bar{X}=9 ; \mathrm{EPM}=1)$ e Farmácia $(\bar{X}=2 ; \mathrm{EPM}=$ 1), Fonoaudiologia $(\bar{X}=9 ; \mathrm{EPM}=1)$ e Ciências Farmacêuticas diurno $(\bar{X}=2.50 ; \mathrm{EPM}=$ $1 ; 50)$, Fonoaudiologia $(\bar{X}=9 ; \mathrm{EPM}=1)$ e Medicina $(\bar{X}=1.50 ; \mathrm{EPM}=1.50)$, Fonoaudiologia $(\bar{X}=9 ; \mathrm{EPM}=1)$ e Odontologia $(\bar{X}=2 ; \mathrm{EPM}=0)$, Fonoaudiologia $(\bar{X}=9$; $\mathrm{EPM}=1)$ e Farmácia $(\bar{X}=2 ; \mathrm{EPM}=1), \operatorname{com~}_{(1,943)}=4.15$ e $\mathrm{p}=0$ (Figura 29). 


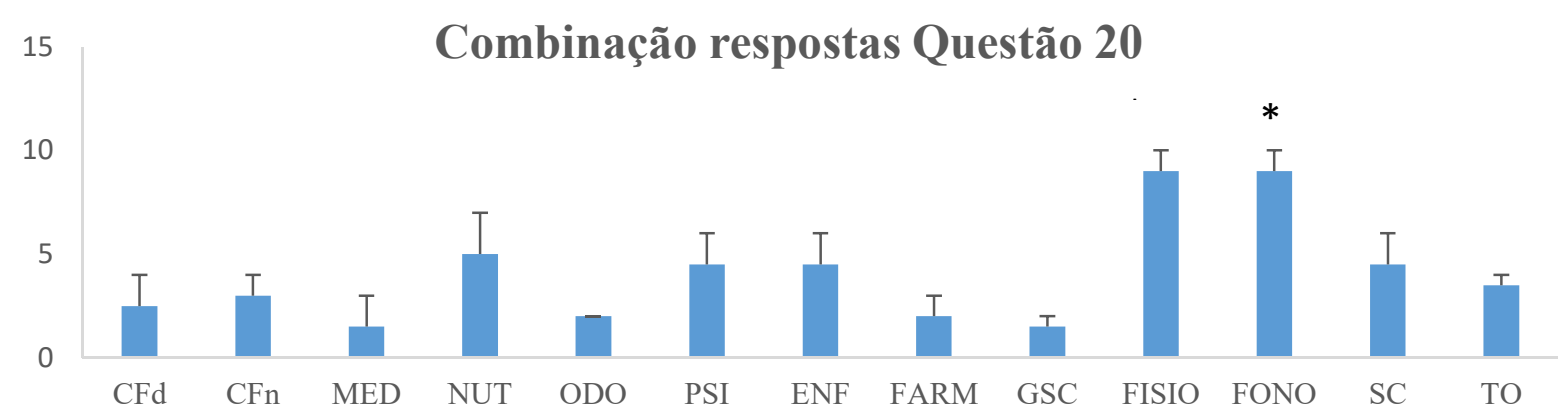

Figura 29: As colunas representam as médias e as barras o EPM da combinação das respostas Alívio de cansaço e Aumentar desempenho de estudo da Questão 20. $\mathrm{p}<0,05$ de acordo com ANOVA One-Way. * Indica diferença significativa de acordo com o teste de StudentNewman-Keuls $(\mathrm{n}$ total $=95)$.

\section{Discussão}

A partir dos resultados obtidos, a discussão foi subdividida em três eixos temáticos, baseados no questionário: conhecimento dos conceitos sobre dependência química, consumo de drogas psicotrópicas entre estudantes e conhecimento sobre o tratamento da dependência química. As perguntas e seus respectivos resultados são agrupados e discutidos segundo o tema que abordam e não necessariamente em ordem numérica.

\section{Conhecimento dos conceitos acerca da dependência química}

A respeito do conhecimento sobre a dependência química por parte dos estudantes, percebe-se uma perspectiva de alteração no conhecimento sobre a temática após cursarem disciplina(s) da área de Farmacologia (Figura 32), pois houve uma tendência ao aumento da média da alternativa Bem informado(a), assim como uma tendência à diminuição das médias das alternativas Mais ou menos informado(a) e Mal informado(a). Similarmente ao estudo de Câmara, Rocha e Balteiro (2011), percebe-se que os estudantes têm consciência do que sabem sobre o assunto, mostrando a relação entre o nível de conhecimento e sua autoavaliação. Apesar disso, na comparação intercursos, a ANOVA One-Way revelou que as maiores médias deram-se para Mais ou menos informado(a), em um curso experimental (Psicologia) e um controle (Fisioterapia), sugerindo que o fato de ter contato com disciplina(s) da área de Farmacologia (no caso, especificamente, Psicofarmacologia), não permite que o estudante se 
autoavalie como detentor do conhecimento nesse assunto (Figura 9). Dos treze cursos avaliados, apenas quatro apresentaram médias superiores por considerarem-se bem informados, dos quais Medicina, Ciências Farmacêuticas noturno, Farmácia e Saúde Coletiva, dado considerado preocupante quando há a expectativa de que futuros profissionais de saúde utilizem seus conhecimentos na intervenção direcionada aos dependentes químicos (Tommasini et al., 2017; Kordahl \& Fougner, 2017).

Da mesma maneira, os dados apontam que o entendimento sobre como deveria se dar o aprendizado a respeito do tema uso de drogas e dependência química durante a graduação (Figura 1) implicam na utilização de outras formas de abordagem da temática, além de aulas expositivas, como congressos, cursos de extensão, palestras e projetos de iniciação científica. Relativamente a esse tema, Ceccin e Feuerwerker (2004, p. 1401) afirmam que "informar o campo das práticas e de uma formação que dê possibilidade a essas práticas" é fundamental para que a área da educação em saúde "cumpra a sua finalidade constitucional de desenvolvimento pleno dos educandos, seu preparo para o exercício da cidadania e sua qualificação para o trabalho". Do mesmo modo, a opinião dos estudantes a respeito da relevância da formação sobre uso de drogas e dependência química em seus cursos (Figuras 41 e 22) indica que eles apontam a relevância da formação na área, algo que sem o qual, de acordo com Vander Bilt et al. (1997), conforme citado por Ramos et al. (2001, p. 36), esses futuros profissionais “não poderiam exercer suas funções dentro do enfoque da prevenção, intervenção e tratamento".

Na comparação entre o antes e depois de cursar alguma(s) disciplina(s) da área de Farmacologia, verifica-se mudança no tipo de material de consulta utilizado quando há dúvidas sobre dependência química, com aumento estatisticamente significativo da média da alternativa Livros e/ou artigos acadêmicos e uma tendência à diminuição da média na alternativa Internet, apesar dessa alternativa ter alcançado a maior média em relação às outras 
alternativas dessa pergunta, tanto na avaliação somente do grupo experimental (Figura 1) como também na avaliação intercursos (Figura 10). A esse respeito, Carraro et al. (2005) defendem que o cuidado profissional dispensado às pessoas envolvidas com drogas está relacionado com a formação recebida durante a graduação e que a formação acadêmica pode não “dar conta de toda esta problemática, mas poderá despertar no futuro profissional a busca de novos conhecimentos e habilidades" (p. 870). Aqui, a questão a ser discutida poderia ser direcionada ao fato de, após cursar disciplina(s) da área de Farmacologia e conhecer os autores/livros, o estudante entraria em contato com a literatura específica da área, sendo essa uma das prerrogativas de qualquer disciplina de graduação mas, em contrapartida, mantém-se em contato com um tipo de literatura, proveniente da internet, cujas informações não condizem, necessariamente, com a aquisição de conhecimento nessa temática. Nessa perspectiva, Griffiths e Mounteney (2017) apontam não somente o uso da internet objetivando a busca de informações mas, também, como um acesso fácil ao consumo de drogas ilícitas. Ainda sobre quem/ou que os estudantes procurariam primeiro em caso de dúvidas, os dados mostram que as alternativas Amigos ou colegas de faculdade e Pais foram recorrentes em todos os cursos (Figura 10), o que demonstra a importância do âmbito pessoal na vida dos estudantes. Esses achados, sobre a fonte de consulta dos estudantes, se associam com aqueles obtidos no estudo de Bucher e Totugui (1987), no qual também foi aplicado um questionário direcionado aos estudantes da Universidade de Brasília, com a mesma temática deste estudo, mostrando que houve procura por colegas e pais para se informar sobre o tema. Esse achado está de acordo com o entendimento de Campos e Figlie (2011) sobre a influência que os indivíduos recebem de seus pais e de seu contexto social, o que explicaria, talvez, a manutenção de conceitos e preconceitos no que tange ao uso de drogas, apesar da exposição e do acesso ao conhecimento acadêmico. Destarte, o direcionamento ao dependente químico é o mesmo relacionado ao paciente que é diagnosticado com transtornos mentais, conforme 
apontado por Corrigan et al. (2017), envolvendo o que é chamado de "fenômeno do estigma da adição", caracterizado por estereotipagem, preconceito e discriminação. Nesse sentido e, de acordo com a investigação, neste estudo, sobre a escolha de uma palavra que definisse o dependente químico, as mais frequentes e recorrentes em todos os cursos avaliados (Figura 55) foram "doente" e "necessitado" sugerindo, de fato, a aproximação com “o louco".

Considerando ainda a procura de recursos para o entendimento do tema, intervenções educativas e preventivas, como as que estão previstas nos objetivos do Serviço de Orientação ao Universitário (SOU) da UnB, seriam relevantes na qualidade de vida dos estudantes e no apoio aos mesmos. Entretanto, os dados obtidos nesse estudo demonstram que, mesmo presente em todos os campi da Universidade de Brasília, esse serviço, que deveria “contribuir para a construção do desenvolvimento acadêmico e oportunizar reflexões e ações integradas que impactem no processo educativo" (UnB, 2017), não é acessado pelos estudantes com a finalidade de esclarecimento sobre a dependência química (Figuras 1 e 10), tendo obtido a frequência zero em todos os cursos.

No que se refere ao uso de drogas psicotrópicas e, a despeito das médias da aprovação de seu uso uma única vez serem maiores que aquelas da aprovação do uso frequente (Figuras 2, 3, 11 e 14), percebe-se que as médias da desaprovação do uso, agudo ou crônico, são ainda maiores (Figuras 33, 35, 12 e 15), exceto para o uso único (experimentar) e frequente do álcool e da maconha. Nesse sentido, Rosenberg, Bonar, Pavlick e Jones (2012) elucidam que há vários fatores que influenciam na aprovação ou desaprovação do uso de drogas, como o medo de ser pego usando drogas, a compreensão da falta de benefícios advindos do uso de drogas e a violação de preceitos religiosos. Em geral, a média da aprovação do uso de álcool e da maconha foi maior que das outras substâncias listadas nesse estudo o que, provavelmente, tem relação com os altos padrões de consumo de álcool entre universitários do país, de acordo com levantamento realizado pela SENAD (Brasil, 2010a), enquanto as maiores médias de 
desaprovação dizem respeito ao uso das substâncias cocaína e crack. Isso poderia ser explicado por fatores tanto socioculturais como econômicos, entendendo-se que o uso do álcool, principalmente, seria mais aceitável na maioria das sociedades e, além disso, comercializado por um custo reduzido em comparação com outras drogas psicotrópicas, tornando-se atraente para jovens estudantes universitários (Figuras 47 e 48) (Andrabi, Khoddam \& Leventhal, 2017). Ao contrário, os riscos e prejuízos para a saúde envolvendo o uso agudo ou crônico da cocaína e seus derivados são amplamente divulgados, explicando o maior nível de desaprovação dessas drogas (Girczys-Poledniok, Pudlo, Jarzab \& Szimlak, 2016; Voon et al., 2016).

Esse estudo também revelou que os estudantes discernem entre usuário de drogas e dependente químico (Figuras 36 e 17), sendo a diferença apontada em todos os cursos avaliados. Todavia, Alves, Martins e Laranjeira (2011) apontam que, mesmo fazendo a diferenciação teórica entre ambos, relativamente ao diagnóstico da dependência química entre os próprios profissionais da área de saúde, há grande negação, falta de entendimento acerca da necessidade de cuidados, medo das consequências da dependência química, como perda de licença de trabalho ou do status, trabalho em excesso e preconceito sobre a temática, que levam à falta de detecção do problema e às justificativas para a conduta de risco por parte do profissional. Outrossim, em outro estudo, Alves, Vieira, Laranjeira, Vieira e Martins (2012) reiteram que é necessária uma discussão sobre o reconhecimento da dependência química na área de saúde como "uma provável doença associada a fatores ocupacionais" (p. 363) e que, a partir da mudança da compreensão a respeito desse tema, haveria um tratamento mais efetivo e precoce para o público em questão. Portanto, a despeito do reconhecimento da diferença teórica, talvez sua aplicação clínica seja complexa.

A ideia anterior é corroborada quando se avalia o conhecimento dos critérios para o diagnóstico da dependência química. Esse estudo revelou que, apesar dos estudantes 
conhecerem alguns desses critérios, evidencia-se uma visão moralista e retrógada que tenta explicar o uso de drogas psicotrópicas, de acordo com Carraro et al. (2005). A associação da dependência química como falta de vontade do indivíduo, por parte dos estudantes que participaram desse estudo (Figuras 5, 37, 19 e 20) reflete, segundo os autores supracitados, "a falta do preparo teórico [...] e contribui para o julgamento de valores e a estigmatização por parte dos profissionais de saúde" (p. 869). Além disso, novamente aqui, ter cursado a(s) disciplina(s) da área de Farmacologia não promoveu alteração quanto a esse conhecimento, não havendo diferença entre os cursos do grupo experimental e do controle. O mesmo foi verificado quanto aos critérios para se considerar a dependência química, tendo sido apontada por cursos de ambos os grupos somente a alternativa Dificuldade de controlar o consumo da substância como estatisticamente significativa (Figura 19). Os estudantes deveriam conhecer a relação da dependência química com bases biológicas, psicológicas e sociais que são independentes da vontade do indivíduo de se manter abstinente, defendem Kabli, Liu, Seifert e $\operatorname{Arnot}(2013)$.

Tomados em conjunto, os resultados das médias referentes às características da dependência química (Figuras 5, 37, 18 e 19) indicam que os estudantes as conhecem, mesmo aqueles que não cursaram a(s) disciplina(s) da área de Farmacologia. Quanto a isso, Pillon et al. (2011a) asseveram que a formação na área deveria envolver múltiplos instrumentos, condutas e fazeres que se relacionariam com saberes e práticas de diversos profissionais, tais como médicos, enfermeiros, psicólogos, fisioterapeutas, terapeutas ocupacionais, nutricionistas, farmacêuticos, entre outros. Nesse sentido, uma alternativa apontada por Tai, Canny, Haines e Molloy (2016) para o desenvolvimento do ensino na área de saúde seria o engajamento, tanto de educadores como de estudantes, na chamada aprendizagem por pares, caracterizada por reduzir a noção de inferioridade e de insegurança relatados pelos estudantes 
no modelo de ensino tradicional, ao proporcionar intervenções reais em saúde, diminuindo a idealização do papel do profissional dessa área.

\section{Consumo de drogas psicotrópicas entre estudantes}

Os dados desse estudo sobre o consumo de drogas psicotrópicas entre os estudantes da área de saúde da Universidade de Brasília revelaram uma tendência ao aumento das médias entre os cursos depois de cursar disciplina(s) da área de Farmacologia (Figura 42). A respeito desse achado, Silva et al. (2006) esclarecem que o consumo de drogas psicotrópicas é maior nos últimos anos da faculdade e que os principais motivos para o uso seriam o estresse no final do curso, a sobrecarga de plantões, estágios e exames finais e a facilidade de obtenção de receitas e do próprio medicamento pelos estudantes. A propósito, conforme Nóbrega et al. (2012), o uso de drogas psicotrópicas pode mudar o entendimento dos estudantes da área de saúde sobre a dependência química, banalizando sua atuação no aconselhamento de seus futuros clientes sobre o uso de drogas e impactando em sua performance estudantil e, além do mais, esses autores sugerem o desenvolvimento de estratégias de diminuição da vulnerabilidade dos estudantes, como programas de prevenção e intervenções educacionais.

Ainda sobre essa temática, a análise da comparação entre todos os cursos (Figura 22) revelou que as substâncias mais consumidas, pelo menos uma vez na vida, entre os estudantes dos cursos avaliados nesse estudo foram: álcool (nos cursos de Psicologia, Fisioterapia e Enfermagem), anfetamina (no curso de Farmácia), nicotina (no curso de Farmácia) e esteroides anabolizantes (no curso de Nutrição). Similarmente, o levantamento da SENAD (Brasil, 2010a) demonstrou que álcool, tabaco e anfetaminas são as três drogas psicotrópicas mais consumidas no meio universitário e ainda, segundo a pesquisa sobre Consumo de Drogas no Mundo (UNODC, 2016), 29 milhões de pessoas ao redor do mundo apresentam transtornos relacionados ao consumo de drogas psicotróicas e, entre essas, as anfetaminas são as drogas mais consumidas, depois da maconha. Nesse sentido, talvez o fato de cursar 
disciplina(s) da área de Farmacologia ou de ser estudante da área de saúde não alterou os padrões de consumo dessas drogas na amostra desse estudo, visto que o álcool e a nicotina, “apesar de serem drogas, não são vistas como tal pela sociedade" e têm seu uso estimulado por meio de propagandas e do fácil acesso, reconhecem Portugal et al. (2008).

No que concerne ao uso de drogas e ao sexo, deve-se levar em consideração que a maioria dos estudantes que participaram desse estudo são do sexo feminino (Figuras 49 e 50). Isso posto, Cunha, Peuker e Bizarro (2012) declaram que o consumo de álcool teria aumentado entre as mulheres e que há incidência de consumo de risco, tanto entre jovens do sexo feminino quanto do masculino, sendo esse consumo de risco definido como o índice elevado de problemas relacionados ao beber. Os achados do presente estudo corroboram com o levantamento de Nealis, Collins, Lee-Baggley, Sherry e Stewart (2017), a respeito da frequência do consumo de bebidas alcóolicas entre as estudantes de graduação ao longo do tempo, e com a revisão de Davoren, Demant, Shiely e Perry (2016), que aponta altos níveis de consumo de álcool entre estudantes universitárias em comparação com estudantes do sexo masculino. Apesar disso, sabe-se que os homens seriam mais vulneráveis ao uso abusivo de drogas psicotrópicas, principalmente de álcool, podendo levar à dependência química, devido ao seu metabolismo mais acelerado comparado às mulheres, explicado pela maior concentração do citocromo P-450 e de testosterona (Oga, Camargo \& Batistuzzo, 2014), o que poderia predispô-los à tolerância, considerado um dos critérios para o diagnóstico de dependência química.

Os achados desse estudo, na comparação entre Antes e Depois de cursar a(s) disciplina(s) da área de Farmacologia, apontam que, entre os motivos para o uso de drogas citados pelos estudantes, a alternativa Melhorar desempenho sexual obteve diferença significativa (Figura 7), ao passo que, na comparação intercursos, as alternativas que tiveram diferença significativa foram Perder a inibição e Porque amigos ou namorado(a) usam 
(Figura 25). A respeito dessas razões para o uso de drogas psicotrópicas, associadas com a as altas médias do uso de álcool, em todos os cursos, Adan, Navarro e Forero (2016) afirmam que o consumo problemático de bebidas alcóolicas se relaciona com problemas emocionais entre as mulheres e dificuldade de controle de impulso entre os homens, além de transtornos emocionais, tensão, preocupação, medo, agir sem pensar e busca por emoção e experiências novas, enquanto Pedrosa, Camacho, Passos e Oliveira (2011) reafirmam a influência do contexto em que os estudantes estão inseridos sobre o uso de drogas. As frequências do uso de drogas psicotrópicas entre os estudantes que participaram desse estudo no último mês e no último ano, retratadas nas Figuras 42 e 43, demonstram que, eventualmente, o consumo não seria esporádico, ao mesmo tempo que na comparação intercursos (Figura 23) a alternativa Sim, acerca do consumo no último ano, apresentou diferença significativa entre os cursos Psicologia e Odontologia. A maior frequência de consumo de drogas psicotrópicas teria relação com a diminuição do acesso a atividades agradáveis e atividades sem a presença de substâncias poderiam ser úteis na prevenção do abuso em jovens, argumentam Andrabi et al. (2017). Ainda nesse sentido, Carvalho e Martins et al. (2012) alegam que o consumo de drogas psicotrópicas entre estudantes universitários, bem como o acesso fácil e rápido às drogas, é uma realidade preocupante.

\section{Conhecimento sobre tratamento da dependência química}

Quanto ao conhecimento acerca do tratamento da dependência química por parte dos estudantes, os dados revelam que os grupos experimental e controle apresentaram alta frequência na alternativa Tem cura (Figura 18), mostrando um lapso no conhecimento sobre a expectativa clínica para a dependência química. Nesse caso, tanto a(s) disciplina(s) da área de Farmacologia, como as outras disciplinas que abordam o uso de drogas, mencionadas na Questão 14 (Figuras 39 e 51), poderiam ter oferecido subsídios teóricos a fim de minimizar esse tipo de falha, o que não ocorreu. O mesmo também foi verificado quando a combinação 
mais frequente entre as alternativas Tem tratamento e Tem cura foi avaliada (Figura 27), sendo os cursos de Fisioterapia (grupo controle) e Enfermagem (grupo experimental) os que alcançaram as maiores médias. A esse respeito, van den Brink e Haasen (2006), Verrando, Robaeys, Matheï e Buntinx (2005) e van den Brink e van Ree (2003) apontam que a dependência química, especialmente nesses estudos que retratam o uso de opióides e cocaína, é uma doença crônica, para o qual não se almeja a cura, mas sim tratamentos que objetivam estabilizar os pacientes e reduzir os danos, aumentando, com isso, sua expectativa e qualidade de vida.

Não obstante, é importante observar que, entre as modalidades de tratamento para a dependência química, a alternativa Uso de medicamentos indicou tendência de aumento, após os estudantes cursarem a(s) disciplina(s) da área de Farmacologia (Figura 45). Esse dado demonstra que o tratamento medicamentoso da dependência química fora abordado durante as aulas. Entrementes, na comparação entre todos os cursos, a alternativa Psicoterapia apresentou sua maior média no curso Psicologia (Figura 26). Tal resultado pode ser pensado em termos da maior exploração do tema nesse curso. Na tentativa de compreensão a respeito das modalidades de tratamento para a dependência química, van Wormer e Davis (2016) declaram que o primeiro elemento para a reabilitação é o reconhecimento do diagnóstico, seguido do tratamento mais adequado, de acordo com cada caso, e sem implicar necessariamente na abstinência, mas na estabilização e no bem-estar do paciente.

No que se refere à modalidade denominada redução de danos, houve diferença significativa com o aumento do conhecimento do termo e a diminuição do desconhecimento depois de cursar a(s) disciplina(s) de Farmacologia (Figura 46). Esse achado foi comprovado pela diminuição estatisticamente significativa da alternativa Não sei depois de cursar a(s) disciplina(s) de Farmacologia (Figura 8). Todavia, na comparação intergrupos, nenhuma das alternativas concernentes à redução de danos da Questão 24 apresentou diferença significativa 
(Figura 54 e 55), denotando que o conhecimento dessa modalidade de tratamento específica seria superficial. O conhecimento adequado acerca da redução de danos poderia reduzir a probabilidade de resultados negativos durante e após o consumo de drogas psicotrópicas por parte dos estudantes, uma vez que a probabilidade de danos biomédicos e psicossociais diminuiriam em função da formação recebida, o que também contribuiria para o desenvolvimento de práticas mais efetivas e centradas no paciente, além de aumentar a aceitação da não-abstinência por parte dos estudantes, asseguram Rosenberg et al. (2011), Reiman (2009) e Davis e Lauritsen (2015).

Finalmente, os achados desse estudo apontam que o fato de cursar a(s) disciplina(s) da área de Farmacologia não alterou os parâmetros avaliados. Ademais, na comparação intercursos verificou-se que, a despeito da formação diversificada entre os cursos da área de saúde, a opinião pessoal e o contexto no qual os estudantes estão inseridos apresentam relevância nessa temática.

\section{Referências}

Adan, A.; Navarro, J. F. \& Forero, D. A. (2016). Personality profile of binge drinking in university students is modulated by sex. A study using the Alternative Five Factor Model. Drug and alcohol dependence, 165: 120-125.

Alves, H. N. P.; Laranjeira, R. R. \& Martins. (2011). Dependência química no currículo de graduação de profissionais da saúde. In: Diehl, A.; Cordeiro, D. \& Laranjeira, R. Dependência química: prevenção, tratamento e políticas públicas. Porto Alegre: Artmed.

Alves, H. N. P.; Vieira, D. L.; Laranjeira, R. R.; Vieira, J. E. \& Martins L. A. N. (2012). Perfil clínico e demográfico de anestesiologistas usuários de álcool e outras drogas atendidos em um serviço pioneiro no Brasil. Revista Brasileira de Anestesiologia, 62 (3): 356-364.

American Psychiatric Association. (2013). Manual diagnóstico e estatístico de transtornos mentais. 5. ed. Porto Alegre: Artmed. 
Andrabi, N.; Khoddam, R. \& Leventhal, A. M. (2017). Socioeconomic disparities in adolescent substance use: role of enjoyable alternative substance-free activities. Social Science and Medicine, 12 (32): 30712-2.

Boni, R., Pillon, S. C.; Santos, E. C.; Camata, M. W.; Macieira, M. S. (2004). Os conteúdos álcool e drogas no ensino de enfermagem da UFES: uma análise crítica. Revista Eletrônica de Enfermagem, 6 (1): 1-15.

Brasil. (2004). Ministério da Saúde (Secretaria de Gestão do Trabalho e da Educação na Saúde - Departamento de Gestão da Educação na Saúde). AprenderSUS: o SUS e os cursos de graduação da área da saúde. Brasília.

Brasil. (2010a). Presidência da República. Secretaria Nacional de Políticas sobre Drogas. I Levantamento Nacional sobre o Uso de Álcool, Tabaco e Outras Drogas entre Universitários das 27 Capitais Brasileiras. GREA/IPQ-HC/FMUSP. Brasília: SENAD.

Brasil. (2010b). VI Levantamento Nacional sobre o Consumo de Drogas Psicotrópicas entre Estudantes do Ensino Fundamental e Médio das Redes Pública e Privada de Ensino nas 27 Capitais Brasileiras. CEBRID/UNIFESP. Brasília: SENAD.

Bucher, R. \& Totugui, M. L. (1987). Conhecimento e uso de drogas entre alunos de Brasília. Psicologia: Teoria e Pesquisa, 3 (2): 178-194.

Câmara, H.; Rocha, C. \& Balteiro, J. (2011). Grau de conhecimento e consumo de psicofármacos dos alunos da Escola Superior de Tecnologia da Saúde de Coimbra. Revista Portuguesa de Saúde Pública, 29 (2): 173-179.

Campos, G. M. \& Figlie, N. B. (2011). Prevenção ao uso nocivo de substâncias focada no indivíduo e no ambiente. In: Diehl, A., Cordeiro, D. C., \& Laranjeira, R. Dependência química: prevenção, tratamento e políticas públicas. Porto Alegre: Artmed, 2011, p. $482-492$.

Carlini, E. A.; Galduróz, J. C.; Noto, A. R.; Carlini, C. M.; Oliveira, L. G.; Nappo, S. A.; Moura, Y. G. \& Sanchez, Z. V. D. M. (2007). II Levantamento Domiciliar sobre o Uso de Drogas Psicotrópicas no Brasil: estudo envolvendo as 108 maiores cidades do país. v. 1. São Paulo: Páginas \& Letras.

Carvalho e Martins, M. C.; Souza Filho, M. D.; Santos, T. L.; Sousa, L. G.; Carvalho, I. L. N. F.; Silva, R. O.; Veloso Junior P. H. V. \& Andrade, F. T. (2012). Uso de drogas 
psicotrópicas entre os estudantes de uma universidade pública. Brasília Médica, 49 (3): 150-157.

Carraro, T. E.; Rassool, G. H. \& Luis, M. A. V. (2005). A formação do enfermeiro e o fenômeno das drogas no sul do Brasil: atitudes e crenças dos estudantes de enfermagem sobre o cuidado. Revista Latino Americana de Enfermagem, 13: 863-871.

CASA. (2007). Wasting the Best and the Brightest: substance abuse at America's Colleges and Universities. Nova Iorque.

Ceccin, R. B. \& Feuerwerker, L. C. M. (2004). Mudança na graduação das profissões de saúde sob o eixo da integralidade. Cadernos de Saúde Pública, 20 (5): 1400-1410.

Correio Braziliense (2016). Ocupação Universidade de Brasília. Recuperado em 20 dezembro 2016 de: http://www.correiobraziliense.com.br/app/noticia/euestudante/ensino_ensinosuperior/2016/10/31/ensino_ensinosuperior_interna,555533/alu nos-da-unb-estao-neste-momento-em-assembleia-geral.shtml e http://www.correiobraziliense.com.br/app/noticia/cidades/2016/12/09/interna_cidadesdf, 560618/desocupacao-na-unb-sera-gradual-e-so-deve-terminar-em-13-dedezembro.shtml.

Corrigan, P.; Schomerus, G.; Shuman, V.; Kraus, D.; Perlick, D.; Harnish, A.; Kulesza, M.; Kane-Willis, K.; Qin, S.; Smelson, D. (2017). Developing a research agenda for understanding the stigma of addictions Part I: lessons from the mental health stigma literature. The American Journal on Addictions, 26 (1): 59-66.

Cunha, S. M.; Peuker, A.C. \& Bizarro, L. (2012). Consumo de álcool de risco e repertório de habilidades sociais entre universitários. Psico, 43 (3), 289 -297.

Davis, A. K. \& Lauritsen, K. J (2015). Acceptability of non-abstinence goals among students enrolled in addiction studies programs across the United States. Substance Abuse, 0 (0): $1-5$.

Davoren, M. P.; Demant, J.; Shiely, F. \& Perry, I. J. (2016). Alcohol consumption among university students in Ireland and the United Kingdom from 2002 to 2014: a systematic review. BMC Public Health, 16: 1-13

Diehl, A.; Cordeiro, D. \& Laranjeira, R. (2010). Tratamentos farmacológicos para dependência química: da evidência científica à prática clínica. Porto Alegre: Artmed. EMCDDA. (2014). European Drug Report: trends and developments. Lisboa. 
IBGE. (2012). Pesquisa nacional de saúde do escolar. Brasil.

Girczys-Poledniok, K.; Pudlo, R.; Jarzab, M.; Szimlak, A. (2016). Cocaine - characteristics and addiction. Medycyna Pracy, 67 (4): 537-544.

Gonzaléz, A. D. \& Almeida, M. J. (2010). Movimentos de mudança na formação em saúde: da medicina comunitária às diretrizes curriculares. Physis Revista de Saúde Coletiva, 20, $2-10$.

Griffiths, P. \& Mounteney, J. (2017). Disruptive Potential of the Internet to Transform Illicit Drug Markets and Impact on Future Patterns of Drug Consumption. Clinical Pharmacology and Therapeutics, 101 (2): 176-178.

Gunther, H. (2003). Como elaborar um questionário. Série: planejamento de pesquisa nas ciências sociais, 1.Laboratório de Psicologia Ambiental, UnB.

Kabli, N.; Liu, B.; Seifert, T. \& Arnot, M. (2013). Effects of academic service learning in drug misuse and addiction on students' learning preferences and attitudes toward harm reduction. American Journal of Pharmaceutical Education, 12, 77(3): 63.

Kordahl, H.L.; Fougner, M. (2017). Facilitating awareness of philosophy of science, ethics and communication through manual skills training in undergraduate education. Physiotherapy Theory and Practice, 17: 1-12.

Kuo, M.; Adlaf, E.M.; Lee, H.; Gliksman, L.; Demers, A. \& Wechsler, H. (2002). More Canadian students drink but American students drink more: comparing college alcohol use in two countries. Addiction, 97 (12): 1583-92.

Lucas, A.; Parente, R.; Picanço, N.; Conceição, D.; Costa, K.; Magalhães, I. \& Siqueira, J. (2006). Uso de psicotrópicos entre universitários da área da saúde da Universidade Federal do Amazonas, Brasil. Cadernos de Saúde Pública, 22 (3): 663-671.

Martins, M; Souza Filho, M; Santos, T.; Sousa, L; Carvalho, I; Silva, R.; Veloso Júnior, P. \& Andrade, F. (2012). Uso de drogas psicotrópicas entre os estudantes de uma universidade pública. Brasília Médica, 49 (3): 150-157.

Medeiros, S.; Rediess, S.; Hauck Filho, N.; Martins, M. \& Mazoni, C. (2012). Prevalência do uso de drogas entre acadêmicos de uma universidade particular do sul do Brasil. Aletheia, 38-39: 81-93. 
Nealis, L. J.; Collins, J. L., Lee-Baggley, D. L.; Sherry, S. B.\& Stewart, S. H. (2017). One of these things is not like the others: Testing trajectories in drinking frequency, drinking quantity, and alcohol-related problems in undergraduate women. Addictive Behaviors, 66: 66- 69.

Nóbrega, M.; Simich, L.; Strike, C.; Brands, B.; Giesbrecht, N. \& Khenti, A. (2012).

Policonsumo simultâneo de drogas entre estudantes de graduação da área de ciências da saúde de uma universidade: implicações de gênero, sociais e legais, Santo André Brasil. Texto e Contexto em Enfermagem, 21: 25-33.

Oga, S.; Camargo, M. M. A. \& Batistuzzo, J. A. O. (2014). Toxicocinética. In: Oga, S.; Camargo, M. M. A. \& Batistuzzo, J. A. O. (eds.). Fundamentos de Toxicologia, São Paulo: Atheneu.

OMS. (2006). Classificação de transtornos mentais e de comportamento da CID-10. Porto Alegre: Artmed.

Pedrosa, A. A. S; Camacho, L. A. B.; Passos, S. R. L. \& Oliveira, R. V. C. (2011). Consumo de álcool entre estudantes universitários. Cadernos de Saúde Pública, 27(8): 1611-1621.

Pillon, S.; Jora, N. P. \& Santos, M. A. (2011a). O papel da equipe multidisciplinar na dependência química. In: Diehl, A.; Cordeiro, D. \& Laranjeira, R. Dependência química: prevenção, tratamento e políticas públicas. Porto Alegre: Artmed, p. 453-460.

Pillon, S.; Siqueira, M. \& Silva, J. (2011b). Dependência química no currículo de graduação de profissionais da saúde. In: Diehl, A.; Cordeiro, D. \& Laranjeira, R. Dependência química: prevenção, tratamento e políticas públicas. Porto Alegre: Artmed, p. 206-212.

Portugal, F.; Souza, R.; Buaiz, V. \& Siqueira, M. (2008). Uso de drogas por estudantes de Farmácia da Universidade Federal do Espírito Santo. Jornal Brasileiro de Psiquiatria, 57 (2): $127-132$.

Ramos, L.; Pillon, S.; Cavalcante, M.; Luiz, M. V.; Padredi, F. M. \& Laranjeira, R. R. (2001). O ensino sobre dependência química em cursos de graduação de enfermagem no Brasil, 1998. Revista Acta Paulista de Enfermagem, 14 (3): 35-43.

Rassool, G. H. (2000). Substance use and misuse one preoccupation of everybody: the responses of health care professional. In: Luís, M. A. V.; Santos, M. A. Uso e abuso de álcool e drogas. FIERP - EERP - USP/FAPESP: Ribeirão Preto, p. 15-26. 
Reiman, A. (2009). Cannabis as a substitute for alcohol and other drugs. Harm Reduction Journal, 6: 35-45.

Rosenberg, H.; Bonar, E. E.; Pavlick, M. \& Jones, L. (2012). Associations between university students' reported reasons for abstinence from illicit substances and type of drug. Journal of College Student Development, 53 (1): 91-105.

Silva, L. V. E. R.; Malbergier, A.; Stempliuk, V. A. \& Andrade, A. G. (2006). Fatores associados ao consumo de álcool e drogas entre estudantes universitários. Revista de Saúde Pública, 40 (2): 280-288.

Soldera, M.; Dalgalarrondo, P.; Corrêa Filho, H. R. \& Silva, C. A. M. (2004). Uso de drogas psicotrópicas por estudantes: prevalência e fatores sociais associados. Revista de Saúde Pública, 38 (2): 277-283.

Tai, J. H.; Canny, B. J.; Haines, T. P. \& Molloy, E. K. (2016). Implementing peer learning in clinical education: a framework to address challenges in the "real world". Teaching and Learning in Medicine, 20: 1-11.

Tockus, D. \& Gonçalves, P. (2008). Detecção do uso de drogas de abuso por estudantes de medicina de uma universidade privada. Jornal Brasileiro de Psiquiatria. 57(3): 184-187.

Tommasini, C.; Dobrowolska, B.; Zarzycka, D.; Bacatum, C.; Bruun, A.M.; Korsath, D.; Roel, S.; Jansen, M.B.; Milling, T.; Deschamps, A.; Mantzoukas, S.; Mantzouka, C. \& Palese, A. (2017). Competence evaluation processes for nursing students abroad: findings from an international case study. Nurse Education Today, 10 (51): 41-47.

UnB. (2015a). Projeto Político Pedagógico: Faculdade de Ceilândia (FCE), Curso de Graduação em Enfermagem. Recuperado em 10 maio 2015 de: http://www.unb.br/aluno_de_graduacao.

UnB. (2015b). Ementas e fluxos dos cursos de Ciências Farmacêuticas, Enfermagem, Farmácia, Fisioterapia, Fonoaudiologia, Gestão em Saúde Coletiva, Medicina, Nutrição, Odontologia, Psicologia, Saúde Coletiva e Terapia Ocupacional. Recuperados em 10 maio 2015 de: http://www.unb.br/aluno_de_graduacao.

UnB. (2017). Serviço de Orientação ao Universitário. Recuperado em 10 janeiro 2017 de: http://www.unb2.unb.br/administracao/decanatos/deg/sou.htm?menu=471.

UNODC. (2014). World Drug Report. Nações Unidas: Nova Iorque. 
UNODC. (2016). World Drug Report. Nações Unidas: Nova Iorque.

van den Brink, W. \& van Ree J. M. (2003). Pharmacological treatments for heroin and cocaine addiction. European Neuropsychopharmacology Journal, 13 (6): 476-87.

van den Brink, W. \& Haasen, (2006). Evidenced-based treatment of opioid-dependent patients. The Canadian Journal of Psychiatry, 51 (10): 635-646.

van Wormer, K. \& Davis, D. R. (2016). Addiction treatment: a strengths perspective. (4. ed.). Brooks Cole: Stanford.

Verrando, R.; Robaeys, G.; Matheï, C. \& Buntinx, F. (2005). Methadone and buprenorphine maintenance therapies for patients with hepatitis $\mathrm{C}$ virus infected after intravenous drug use. Acta Gastroenterologie, 68 (1): 81-85.

Voon, P.; Ti, L.; Dong, H.; Milloy, M. J.; Wood, E.; Kerr, T. \& Hayashi, K. (2016). Risky and rushed public crack cocaine smoking: the potential for supervised inhalation facilities. BMC Public Health, 16: 476. 
Apêndice 


\section{Apêndice}

No Apêndice estão os resultados da análise dos dados cujas respostas não apresentaram diferença significativa. Na primeira parte, os resultados do teste $t$ não-pareado de Student da comparação entre os dados dos 570 estudantes dos cursos Ciências Farmacêuticas (diurno e noturno), Enfermagem, Farmácia, Medicina, Nutrição, Odontologia e Psicologia Antes de cursar a(s) disciplina(s) da área de Farmacologia e Depois.

Assim sendo, na sequência são expostos os resultados das comparações intergrupos, dos 944 questionários oriundos dos cursos Ciências Farmacêuticas (diurno e noturno), Enfermagem, Farmácia, Medicina, Nutrição, Odontologia e Psicologia (grupo experimental), Gestão em Saúde Coletiva, Fisioterapia, Fonoaudiologia, Saúde Coletiva e Terapia Ocupacional (grupo controle), analisados pela Análise de Variância de uma via (ANOVA One-Way). O teste post-hoc usado para identificar as diferenças foi o teste de StudentNewman-Keuls $(\mathrm{p}<0,05)$.

\section{Avaliação das respostas dos questionários antes e após cursar a(s) disciplina(s) da área de Farmacologia:}

A média geral de idade dos 570 participantes analisados no teste $t$ não-pareado de Student foi 24.4 anos (Figura 30), sendo 418 do sexo feminino e 152 do masculino (Figura 31).

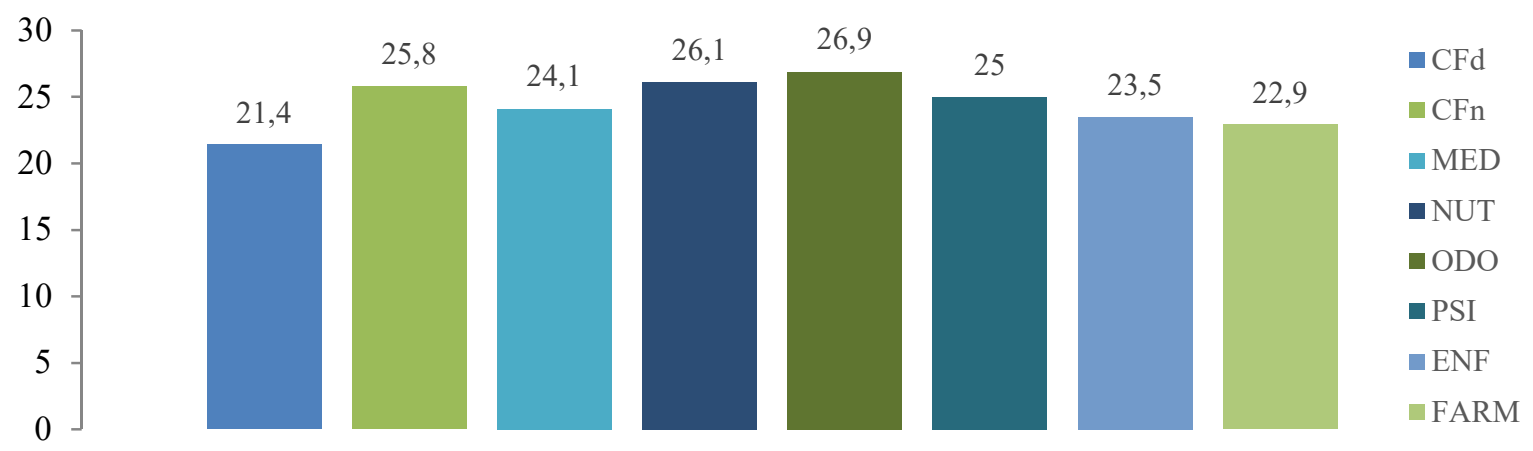

Figura 30: Distribuição da média da idade dos estudantes por curso. Ciências Farmacêuticas diurno (CFd); Ciências Farmacêuticas noturno (CFn); Medicina (Med); Nutrição (Nut); 
Odontologia (Odo); Psicologia (Psi); Enfermagem (Enf) e Farmácia (Farm). As colunas representam os cursos e as barras as médias das frequências.

\section{Distribuição dos estudantes por sexo}

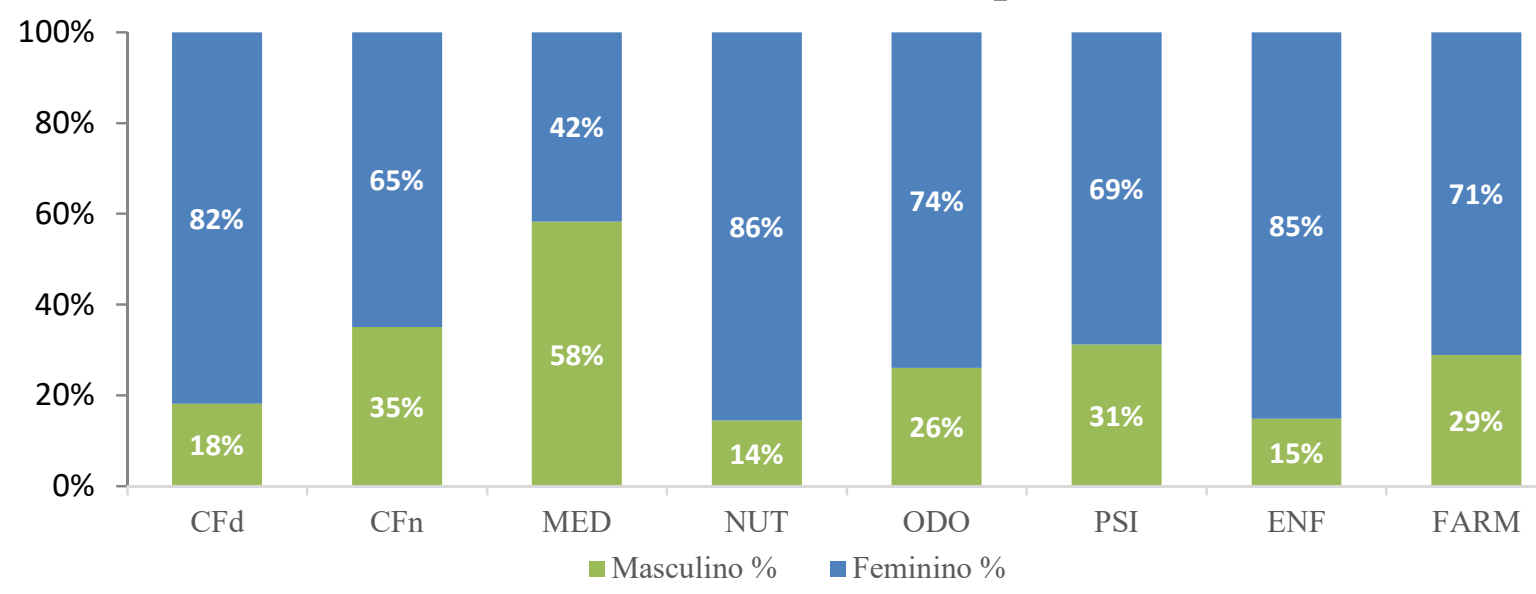

Figura 31: Distribuição da frequência (em \%) dos estudantes por sexo e por curso. Ciências Farmacêuticas diurno (CFd): Feminino (F) $n=63$ e Masculino $(M) n=14$; Ciências Farmacêuticas noturno (CFn): (F) $n=37$ e (M) $n=20$; Medicina (Med): (F) $n=20$ e $M n=$ 28. As colunas representam os cursos e as barras as frequências.

A seguir são apresentados os dados relativos a cada questão.

As Questões 1 e 2 indagavam sobre a idade e o sexo do estudante (Figuras 30 e 31, respectivamente), enquanto a Questão 3 inquiria sobre o curso e teve como função certificar a procedência do estudante, uma vez que em algumas disciplinas nas quais as coletas foram realizadas poderiam haver estudantes de outros cursos. Do mesmo modo, a Questão 4, a respeito do estudante ter cursado ou não disciplina(s) da área de Farmacologia, buscava assegurar a inserção correta do participante ou no grupo Antes ou no grupo Depois de ter cursado tal(is) disciplina(s).

\begin{tabular}{l}
\hline 5. Em relação ao conhecimento sobre o uso de drogas, você \\
se considera (Marque apenas uma alternativa) \\
\hline Bem informado(a) \\
\hline Mais ou menos informado(a) \\
\hline Mal informado(a) \\
\hline
\end{tabular}

Na Questão 5, o teste $t$ não-pareado de Student revelou que não houve diferença significativa entre as respostas obtidas em Bem informado(a) Antes (Média $(\bar{X})=13$; Erro Padrão da Média $(E P M)=1.75)$ e Depois $(\bar{X}=16 ;$ EPM $=2.24), t=-1.05$ e $p=0.30$, assim como as respostas obtidas em Mais ou menos informado(a) Antes $(\bar{X}=21.5 ; \mathrm{EPM}=3.22) \mathrm{e}$ 
Depois $(\bar{X}=18 ; \mathrm{EPM}=1.78), t=0.95$ e $\mathrm{p}=0.35$ e Mal informado(a) Antes $(\bar{X}=2.15 ; \mathrm{EPM}$ $=0.78)$ e Depois $(\bar{X}=1 ; \mathrm{EPM}=0.37), t=1.29 \mathrm{e} \mathrm{p}=0.21$ também não apresentaram diferença significativa (Figura 32).

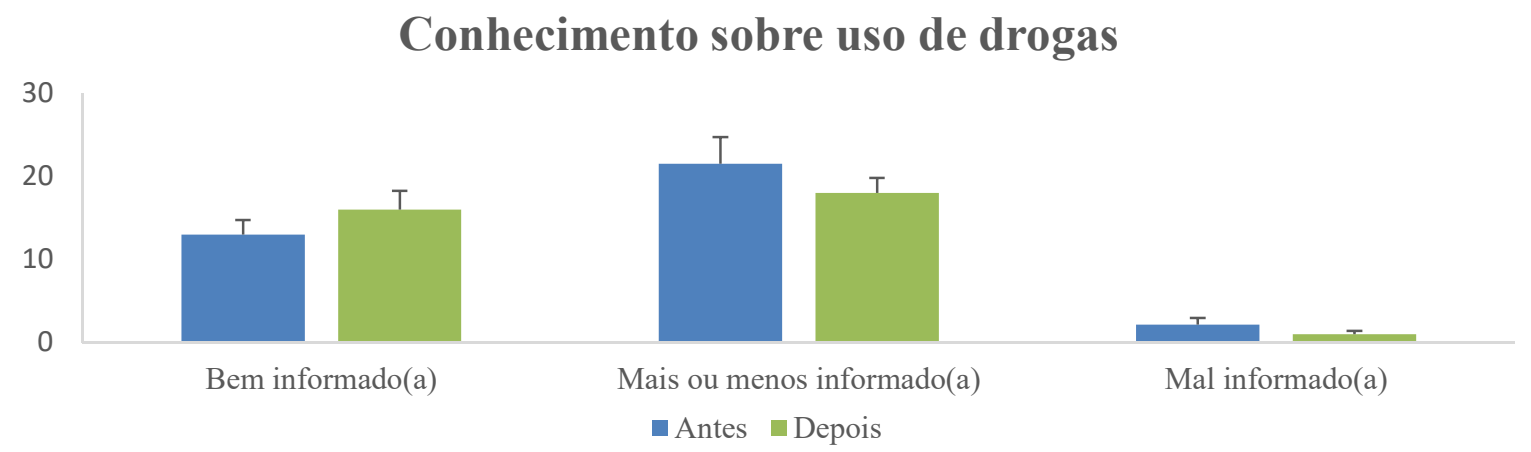

Figura 32: Conhecimento sobre uso de drogas antes e depois de cursar a(s) disciplina(s) da área de Farmacologia. As colunas representam as médias e as barras o EPM. $p<0,05$, de acordo com o teste $t$ não-pareado de Student. Antes $(\mathrm{n}=289)$ e Depois $(\mathrm{n}=281)$.

Quanto à desaprovação do uso uma única vez, o teste $t$ não-pareado de Student não apresentou diferença significativa em nenhuma das alternativas (Figura 33): Álcool Antes ( $\bar{X}$ $=4.13 ; \mathrm{EPM}=0.74)$ e Depois $(\bar{X}=3.25 ; \mathrm{EPM}=0.86), t=0.77$ e $\mathrm{p}=0.45$, Benzodiazepínicos e barbitúricos Antes $(\bar{X}=10.3 ; \mathrm{EPM}=1.22)$ e Depois $(\bar{X}=10.8 ; \mathrm{EPM}=$ $0.90), t=-0.32$ e p $=0.74$, Cigarro de nicotina Antes $(\bar{X}=24.5 ; \mathrm{EPM}=2.71)$ e Depois $(\bar{X}=$ 20.6; $\mathrm{EPM}=2.32), t=1.09$ e $\mathrm{p}=0.29$, Cigarro de Maconha Antes $(\bar{X}=19.4 ; \mathrm{EPM}=2.58) \mathrm{e}$ Depois $(\bar{X}=18.4 ; \mathrm{EPM}=2.80), t=0.26$ e $\mathrm{p}=0.79$, Cocaina Antes $(\bar{X}=34 ; \mathrm{EPM}=4.31) \mathrm{e}$ Depois $(\bar{X}=24.3 ; \mathrm{EPM}=5.19), t=1.45 \mathrm{e} \mathrm{p}=0.17$, Crack Antes $(\bar{X}=35.5 ; \mathrm{EPM}=4.44) \mathrm{e}$ Depois $(\bar{X}=26 ; \mathrm{EPM}=5.84), t=1.29$ e $\mathrm{p}=0.21$, Drogas sintéticas Antes $(\bar{X}=25.4$; EPM $=2.74)$ e Depois $(\bar{X}=24.1 ; \mathrm{EPM}=3.08), t=0.30 \mathrm{e} \mathrm{p}=0.76$, Esteroides anabolizantes Antes $(\bar{X}=30.5 ; \mathrm{EPM}=4.10)$ e Depois $(\bar{X}=20.1 ; \mathrm{EPM}=5.56), t=1.50$ e $\mathrm{p}=0.15$ e Inalantes Antes $(\bar{X}=29,9 ; \mathrm{EPM}=3.05)$ e Depois $(\bar{X}=26.6 ; \mathrm{EPM}=2.27), t=0.85$ e $\mathrm{p}=0.40$. 


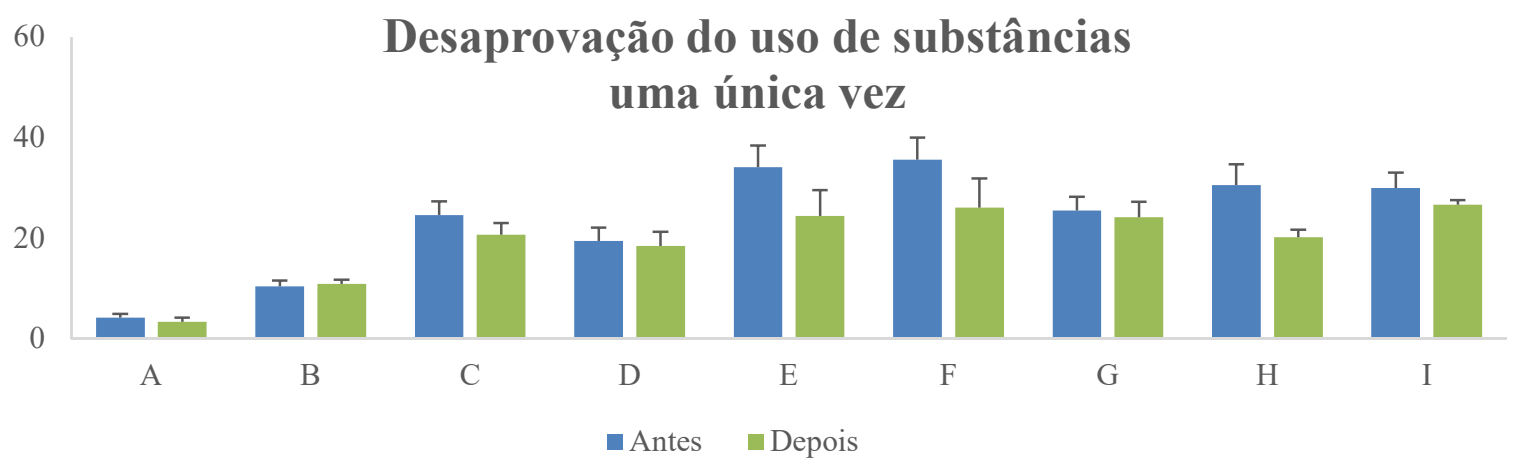

Figura 33: Desaprovação do uso de substâncias uma única vez antes e depois de cursar a(s) disciplina(s) da área de Farmacologia. As colunas representam as médias das alternativas $\mathrm{A}=$ Álcool, $\mathrm{B}=$ Benzodiazepínicos e barbitúricos, $\mathrm{C}=$ Cigarro de nicotina, $\mathrm{D}=$ Cigarro de maconha, $\mathrm{E}=$ Cocaína, $\mathrm{F}=$ Crack, $\mathrm{G}=$ Drogas sintéticas, $\mathrm{H}=$ Esteroides anabolizantes, $\mathrm{I}=$ Inalantes e as barras o EPM. $\mathrm{p}<0,05$, de acordo com o teste $t$ não-pareado de Student. Antes $(\mathrm{n}=289)$ e Depois $(\mathrm{n}=281)$.

Em relação à alternativa não sei, sobre o uso uma única vez, o teste $t$ não-pareado de Student revelou que nenhuma alternativa apresentou diferença significativa (Figura 7) Álcool Antes $(\bar{X}=4.13 ; \mathrm{EPM}=0.97)$ e Depois $(\bar{X}=3.50 ; \mathrm{EPM}=1.01), t=0.44$ e $\mathrm{p}=0.66$, Benzodiazepínicos e barbitúricos Antes $(\bar{X}=20.9$; EPM $=3.40)$ e Depois $(\bar{X}=13.8 ;$ EPM $=$ $2.31), t=1.73$ e $\mathrm{p}=0.10$, Cigarro de nicotina Antes $(\bar{X}=3.25 ; \mathrm{EPM}=0.70)$ e Depois $(\bar{X}=$ 3.13; $\mathrm{EPM}=0.69), t=1.12$ e $\mathrm{p}=0.90$, Cigarro de maconha Antes $(\bar{X}=5.50 ; \mathrm{EPM}=0.94) \mathrm{e}$ Depois $(\bar{X}=4.25 ; \mathrm{EPM}=0.75), t=1.04$ e $\mathrm{p}=0.31$, Cocaína Antes $(\bar{X}=1.75 ; \mathrm{EPM}=0.52)$ e Depois $(\bar{X}=2.75 ; \mathrm{EPM}=1 ; 90), t=-0.62$ e $\mathrm{p}=0.54$, Crack Antes $(\bar{X}=1.38 ; \mathrm{EPM}=0.42)$ e Depois $(\bar{X}=5.88 ; \mathrm{EPM}=3.77), t=-1.18$ e $\mathrm{p}=0.25$, Drogas sintéticas Antes $(\bar{X}=7.13$; $\mathrm{EPM}=1.42)$ e Depois $(\bar{X}=4.88 ; \mathrm{EPM}=0.93), t=1.32$ e $\mathrm{p}=0.20$, Esteroides anabolizantes Antes $(\bar{X}=4.38 ; \mathrm{EPM}=1.40)$ e Depois $(\bar{X}=4.38 ; \mathrm{EPM}=1.39), t=0$ e $\mathrm{p}=1$ e Inalantes Antes $(\bar{X}=5 ; \mathrm{EPM}=1.55)$ e Depois $(\bar{X}=5 ; \mathrm{EPM}=1.05), t=0$ e $\mathrm{p}=1$. 


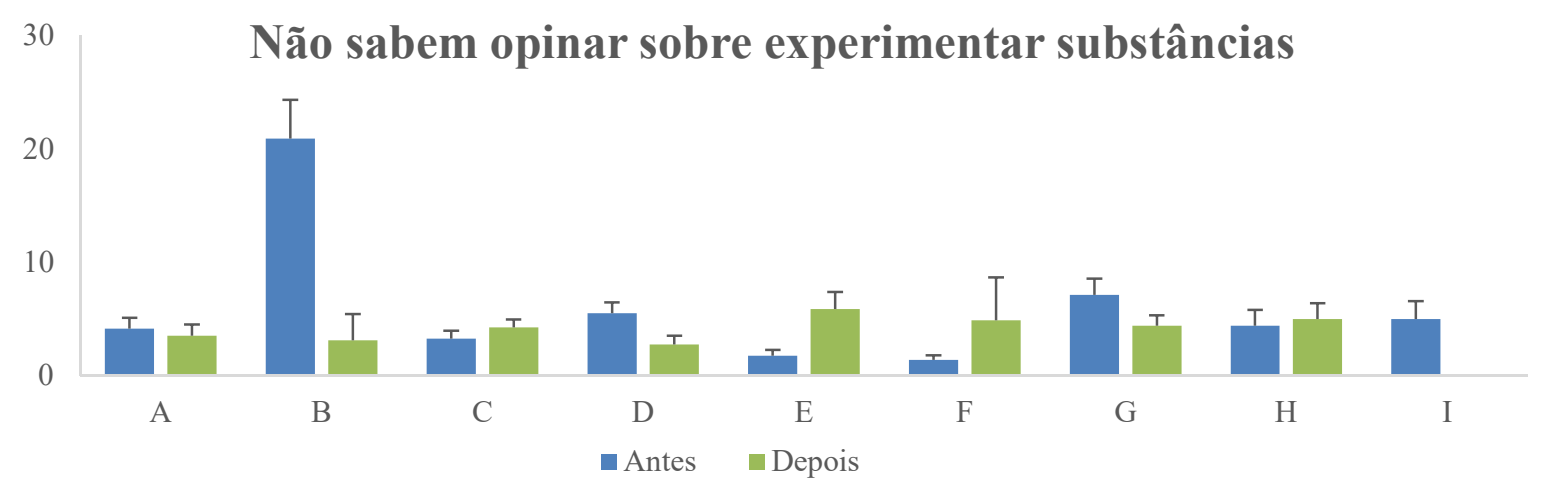

Figura 34: Alternativa não sei sobre experimentar substâncias antes e depois de cursar a(s) disciplina(s) da área de Farmacologia. As colunas representam as médias das alternativas $\mathrm{A}=$ Álcool, $\mathrm{B}=$ Benzodiazepínicos e barbitúricos, $\mathrm{C}=$ Cigarro de nicotina, $\mathrm{D}=$ Cigarro de maconha, $\mathrm{E}=$ Cocaína, $\mathrm{F}=$ Crack, $\mathrm{G}=$ Drogas sintéticas, $\mathrm{H}=$ Esteroides anabolizantes, $\mathrm{I}=$ Inalantes e as barras o EPM. $\mathrm{p}<0,05$ de acordo com o teste $t$ não-pareado de Student. Antes $(\mathrm{n}=289)$ e Depois $(\mathrm{n}=281)$.

Sobre a desaprovação do uso frequente das substâncias da Questão 8, o teste $t$ nãopareado de Student mostrou que não houve diferença significativa em nenhuma das alternativas (Figura 35): Álcool Antes $(\bar{X}=22.8 ; \mathrm{EPM}=3.20)$ e Depois $(\bar{X}=20.6$; EPM $=$ $2.11), t=0.55$ e $\mathrm{p}=0.58$, Benzodiazepínicos e barbitúricos Antes $(\bar{X}=21.3 ; \mathrm{EPM}=2.68) \mathrm{e}$ Depois $(\bar{X}=24.9 ; \mathrm{EPM}=2.38), t=-1.01$ e $\mathrm{p}=0.32$, Cigarro de nicotina Antes $(\bar{X}=31.3$; $\mathrm{EPM}=3.71)$ e Depois $(\bar{X}=31.5 ; \mathrm{EPM}=2.19), t=-0.05$ e $\mathrm{p}=0.95$, Cigarro de maconha Antes $(\bar{X}=28 ; \mathrm{EPM}=3.15)$ e Depois $(\bar{X}=28.4 ; \mathrm{EPM}=2.71), t=0.09$ e $\mathrm{p}=0.92$, Cocaína Antes $(\bar{X}=35.1 ; \mathrm{EPM}=4.46)$ e Depois $(\bar{X}=33.5 ; \mathrm{EPM}=2.61), t=0.31$ e $\mathrm{p}=0.75$, Crack Antes $(\bar{X}=35.3 ; \mathrm{EPM}=4.39)$ e Depois $(\bar{X}=33.9 ; \mathrm{EPM}=2.59), t=0.26$ e $\mathrm{p}=0.79$, Drogas sintéticas Antes $(\bar{X}=30.5 ; \mathrm{EPM}=3.45)$ e Depois $(\bar{X}=31.8 ; \mathrm{EPM}=2.72), t=-0.28$ e $\mathrm{p}=$ 0.78 , Esteroides anabolizantes Antes $(\bar{X}=33.5 ; \mathrm{EPM}=4.46)$ e Depois $(\bar{X}=32.1 ; \mathrm{EPM}=$ $2.48), t=0.26$ e p $=0.79$ e Inalantes Antes $(\bar{X}=32 ; \mathrm{EPM}=3.47)$ e Depois $(\bar{X}=32.1 ; \mathrm{EPM}$ $=2.79), t=-0.02$ e $\mathrm{p}=0.97$. 


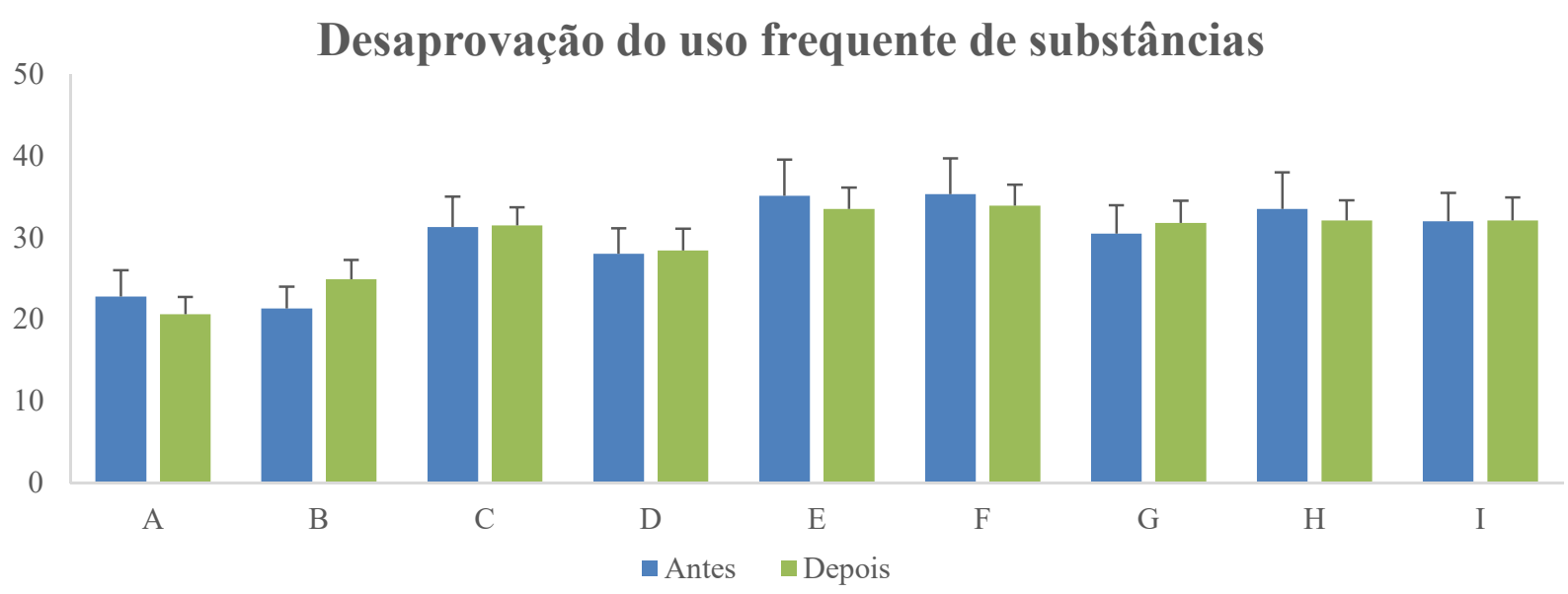

Figura 35: Desaprovação do uso frequente de substâncias antes e depois de cursar a(s) disciplina(s) da área de Farmacologia. As colunas representam as médias das alternativas $\mathrm{A}=$ Álcool, $\mathrm{B}=$ Benzodiazepínicos e barbitúricos, $\mathrm{C}=$ Cigarro de nicotina, $\mathrm{D}=$ Cigarro de maconha, $\mathrm{E}=$ Cocaína, $\mathrm{F}=$ Crack, $\mathrm{G}=$ Drogas sintéticas, $\mathrm{H}=$ Esteroides anabolizantes, $\mathrm{I}=$ Inalantes e as barras o EPM. p $<0,05$, de acordo com o teste $t$ não-pareado de Student. Antes $(\mathrm{n}=289)$ e Depois $(\mathrm{n}=281)$.

\begin{tabular}{l}
$\begin{array}{l}\text { 9. Para você, existe diferença entre usuário de drogas e } \\
\text { dependente químico? }\end{array}$ \\
\hline Sim \\
\hline Não
\end{tabular}

Na Questão 9, o teste $t$ não-pareado de Student revelou que não houve diferença significativa entre as respostas obtidas entre as alternativas $\operatorname{Sim}$ Antes $(\bar{X}=25 ; \mathrm{EPM}=3.94)$ e Depois $(\bar{X}=26.4 ; \mathrm{EPM}=2.28), t=-0.30$ e $\mathrm{p}=0.76$ e Não Antes $(\bar{X}=10.7 ; \mathrm{EPM}=1.06)$ e Depois $(\bar{X}=3.01 ; \mathrm{EPM}=1.33), t=1.10$ e $\mathrm{p}=0.28($ Figura 36$)$

\section{Opinião sobre diferença entre dependente químico e usuário de drogas}

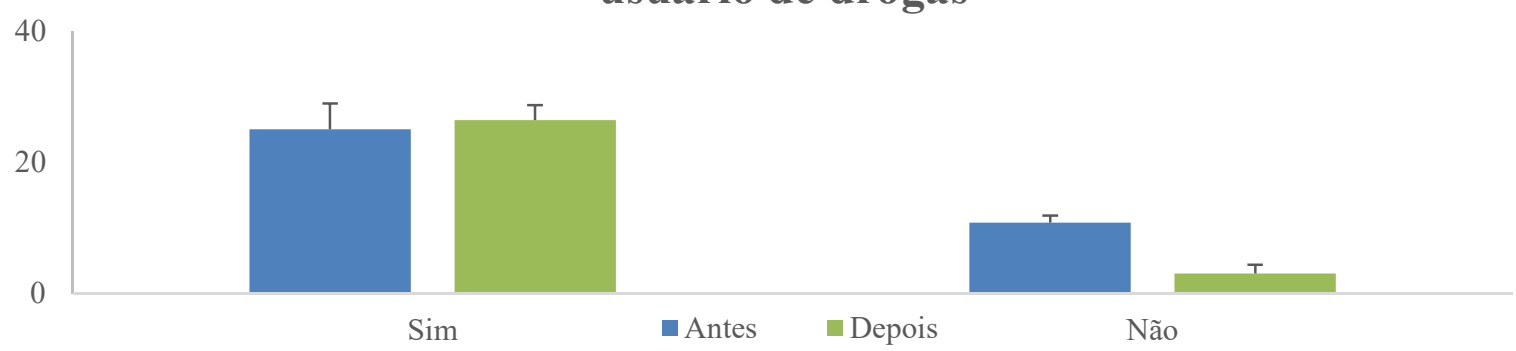

Figura 36: Opinião dos estudantes sobre possível diferença entre dependente químico e usuário de drogas antes e depois de cursar a(s) disciplina(s) da área de Farmacologia. As colunas representam as médias e as barras o EPM. $\mathrm{p}<0,05$, de acordo com o teste $t$ nãopareado de Student. Antes $(\mathrm{n}=289)$ e Depois $(\mathrm{n}=281)$.

11. O que poderia caracterizar a dependência química? (Assinale todas as alternativas que se aplicam)

Abandono dos prazeres e interesses

Estado de abstinência 


\begin{tabular}{l}
\hline Dificuldade de controlar o consumo \\
\hline Fissura \\
\hline Persistência no uso \\
\hline Tolerância \\
\hline Nenhuma das anteriores \\
\hline
\end{tabular}

Na Questão 11, o teste $t$ não-pareado de Student mostrou que não houve diferença significativa entre as respostas obtidas em nenhuma das alternativas acima relacionadas (Figura 37): Abandono dos prazeres e interesses Antes $(\bar{X}=11.6 ; \mathrm{EPM}=1.95)$ e Depois $(\bar{X}$ $=15.5 ; \mathrm{EPM}=1.83), t=-1.45$ e $\mathrm{p}=0.17$, Estado de abstinência Antes $(\bar{X}=16.8 ; \mathrm{EPM}=$ 2.69) e Depois $(\bar{X}=18.6 ; \mathrm{EPM}=2.18), t=-0.54$ e $\mathrm{p}=0.59$, Dificuldade de controlar $o$ consumo Antes $(\bar{X}=31.3 ; \mathrm{EPM}=4.77)$ e Depois $(\bar{X}=30 ; \mathrm{EPM}=2.92), t=0.22$ e $\mathrm{p}=0.82$, Fissura Antes $(\bar{X}=15 ; \mathrm{EPM}=3.18)$ e Depois $(\bar{X}=16.5 ; \mathrm{EPM}=2.87), t=-0.35$ e $\mathrm{p}=0.73$, Persistência no uso Antes $(\bar{X}=23.9 ; \mathrm{EPM}=3.20)$ e Depois $(\bar{X}=22 ; \mathrm{EPM}=2.48), t=0.46$ e $\mathrm{p}=0.65$, Tolerância Antes $(\bar{X}=6.25 ; \mathrm{EPM}=2.34)$ e Depois $(\bar{X}=11 ; \mathrm{EPM}=2.40), t=-$ 1.42 e p $=0.17$ e Nenhuma das anteriores Antes $(\bar{X}=0.62 ; \mathrm{EPM}=0.18)$ e Depois $(\bar{X}=$ $1.25 ; \mathrm{EPM}=0.41), t=-1.39$ e $\mathrm{p}=0.18$

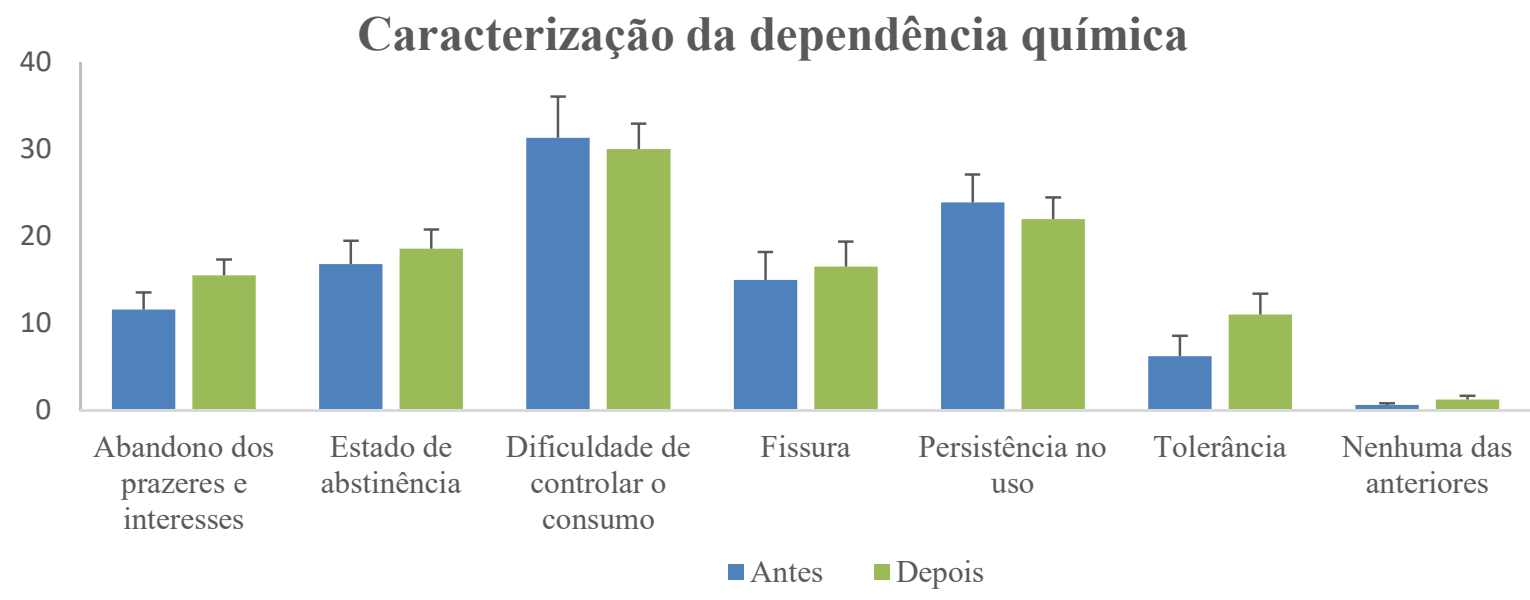

Figura 37: Características da dependência química antes e depois de cursar a(s) disciplina(s) da área de Farmacologia. As colunas representam as médias e as barras o EPM. p < 0,05, de acordo com o teste $t$ não-pareado de Student. Antes $(\mathrm{n}=289)$ e Depois $(\mathrm{n}=281)$.

12. Como você acha que poderia se dar o aprendizado sobre uso de drogas e dependência química durante a graduação? (Assinale todas as alternativas que se aplicam)

Aulas expositivas

Congressos 


\begin{tabular}{l}
\hline Cursos de extensão \\
\hline Palestras \\
\hline Projetos de iniciação científica \\
\hline Outros \\
\hline
\end{tabular}

$\mathrm{Na}$ Questão 12, o teste $t$ não-pareado de Student não mostrou diferença significativa entre as respostas obtidas em nenhuma das alternativas (Figura 38): Aulas expositivas Antes ( $\bar{X}=23.8 ; \mathrm{EPM}=3.72)$ e Depois $(\bar{X}=23.3 ; \mathrm{EPM}=2.74), t=0.10$ e $\mathrm{p}=0.91$, Congressos Antes $(\bar{X}=16.8 ; \mathrm{EPM}=2.83)$ e Depois $(\bar{X}=15.6 ; \mathrm{EPM}=2.59), t=0.29$ e $\mathrm{p}=0.77$, Cursos de extensão Antes $(\bar{X}=18.5 ; \mathrm{EPM}=3.67)$ e Depois $(\bar{X}=19.8 ; \mathrm{EPM}=3.06), t=0.26$ e $\mathrm{p}=$ 0.79 , Palestras Antes $(\bar{X}=26 ; \mathrm{EPM}=3.72)$ e Depois $(\bar{X}=23.1 ; \mathrm{EPM}=1.97), t=0.68$ e $\mathrm{p}=$ 0.50, Projetos de iniciação científica Antes $(\bar{X}=19.6 ; \mathrm{EPM}=3.39)$ e Depois $(\bar{X}=20.4$; $\mathrm{EPM}=2.51), t=-0.17$ e $\mathrm{p}=0.86$ e Outros Antes $(\bar{X}=5.75 ; \mathrm{EPM}=1.69)$ e Depois $(\bar{X}=$ $5.63 ; \mathrm{EPM}=1.10), t=0.06$ e $\mathrm{p}=0.95$.

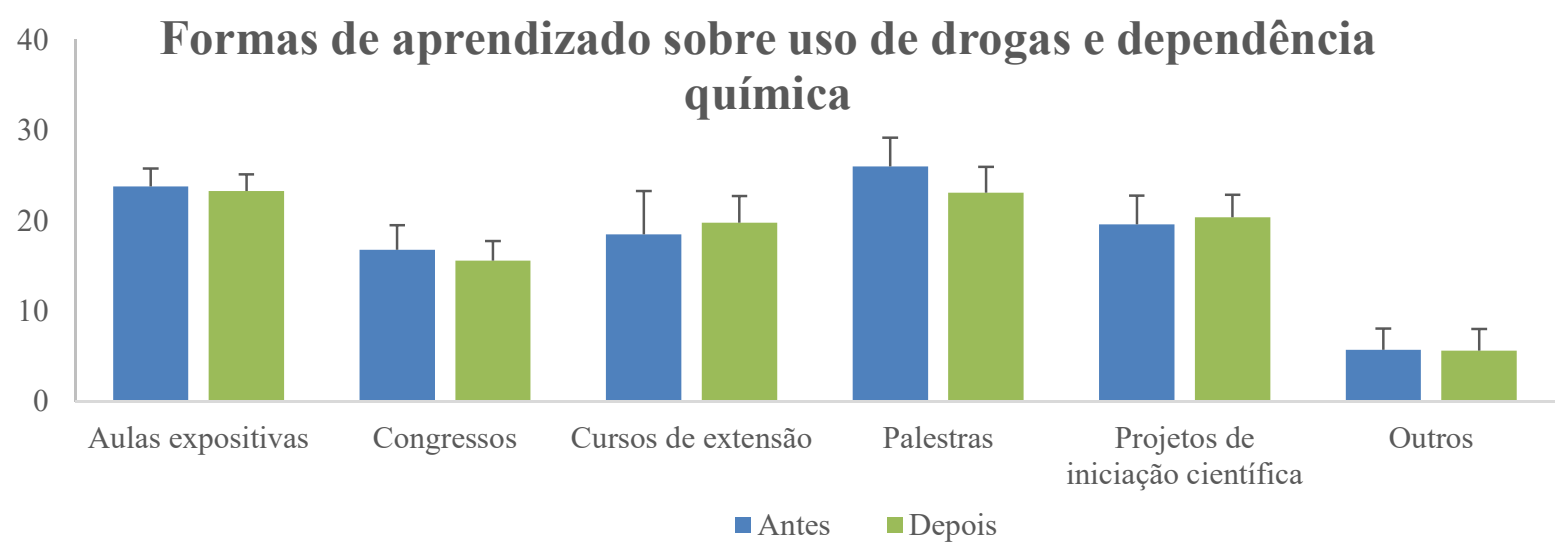

Figura 38: Formas de aprendizado acerca do uso de drogas e da dependência química antes e depois de cursar a(s) disciplina(s) da área de Farmacologia. As colunas representam as médias e as barras o EPM. $\mathrm{p}<0,05$, de acordo com o teste $t$ não-pareado de Student. Antes $(\mathrm{n}=289)$ e Depois $(\mathrm{n}=281)$.

A seguir são apresentadas as frequências das disciplinas mais citadas nos cursos do grupo experimental (Figura 39). 
Disciplinas apontadas por abordarem o uso de drogas no curso Ciências

Farmacêuticas diurno

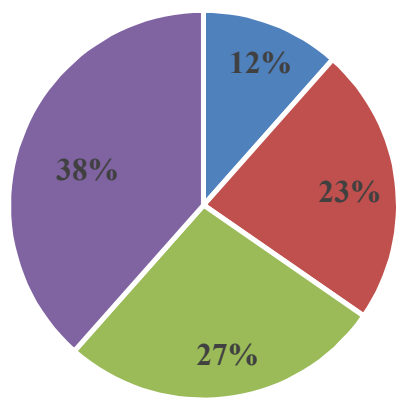

- Farmacologia 2

- Farmacologia

- Toxicologia

- Outros

Disciplinas apontadas por abordarem o uso de drogas no curso Medicina

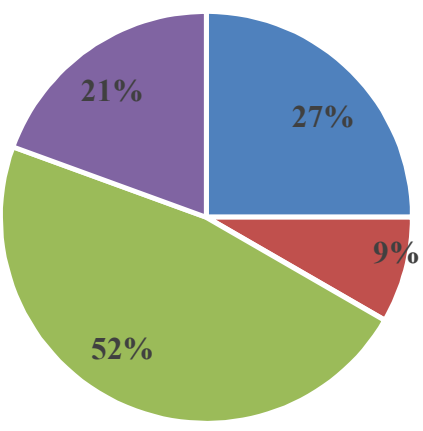

- Farmacologia Médica

- Fundamentos em Farmacologia

- Psiquiatria

- Outros

Disciplinas apontadas por abordarem o uso de drogas no curso Ciências

Farmacêuticas noturno

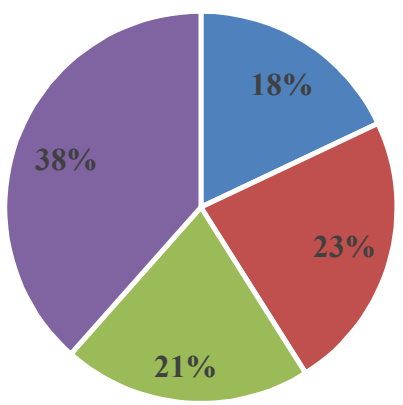

- Farmacologia 2 - Farmacologia

- Toxicologia - Outros

Disciplinas apontadas por abordarem o uso de drogas no curso Nutrição

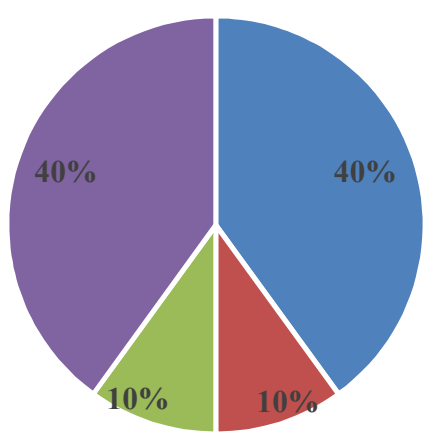

- Farmacologia

- Fisiologia

- Psicologia aplicada à saúde - Outros 
Disciplinas apontadas por abordarem o uso de drogas no curso Odontologia

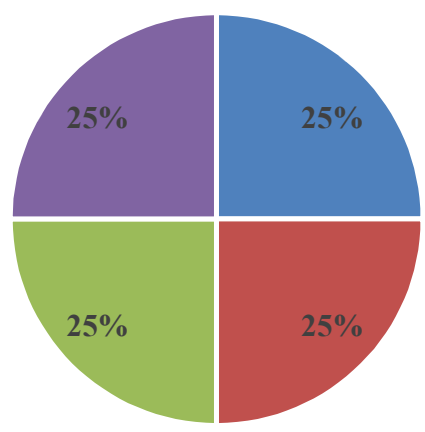

- Farmacologia

- Fisiologia

- Práticas de Saúde Coletiva

- Outros

Disciplinas apontadas por abordarem o uso de drogas no curso Enfermagem

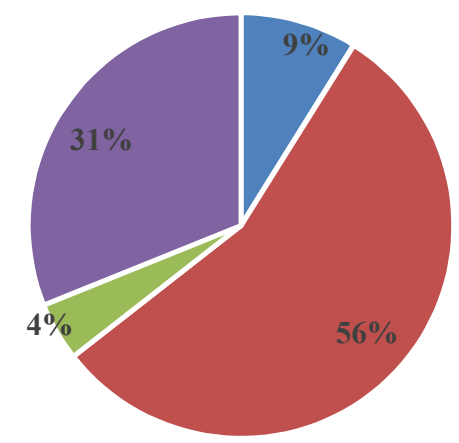

- Saúde e Sociedade

- Cuidado de Enfermagem Psicosocial em Saúde Mental

- Farmacologia

- Outros

\author{
Disciplinas apontadas por \\ abordarem o uso de drogas \\ no curso Psicologia
}

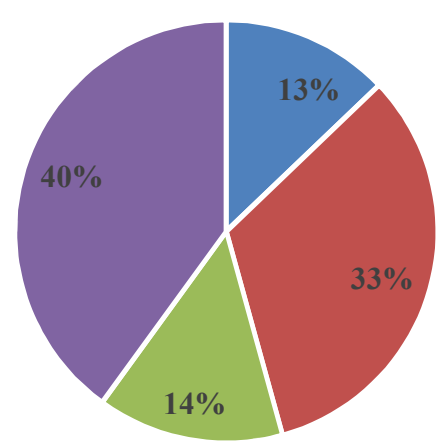

- Fundamentos de Análise do Comportamento

- Fundamentos de Neurociências

- Psicofarmacologia

- Outros

\section{Disciplinas apontadas por abordarem o uso de drogas no curso Farmácia}

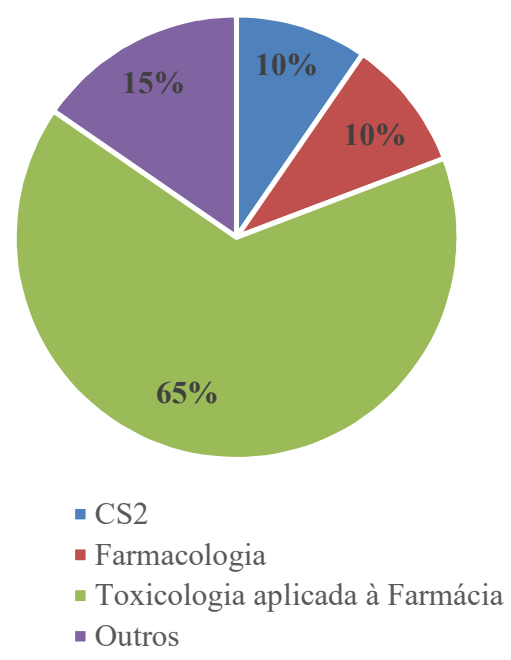

Figura 39: Frequências das disciplinas apontadas por abordarem o uso de drogas e a dependência química mencionadas na Questão 14. As colunas representam as maiores frequências obtidas nos cursos Ciências Farmacêuticas diurno $(\mathrm{n}=18)$, Ciências Farmacêuticas noturno $(n=27)$, Medicina $(n=36)$, Nutrição $(n=17)$, Odontologia $(n=5)$, Psicologia $(n=50)$, Enfermagem $(n=36)$ e Farmácia $(n=44)$. $(n$ total $=233)$. 
A Questão 15 inquiria se a disciplina listada na Questão 14 mudara o entendimento dos estudantes acerca do assunto uso de drogas e dependência química. Posto que o total de respostas obtidas nessa questão não foi numericamente expressivo, uma vez que nem todos os estudantes que responderam o questionário apontaram alguma disciplina que aludisse ao tema, as frequências das alternativas $\operatorname{Sim}$ e Não, obtidas nos cursos do grupo experimental, são apresentadas abaixo (Figura 40).

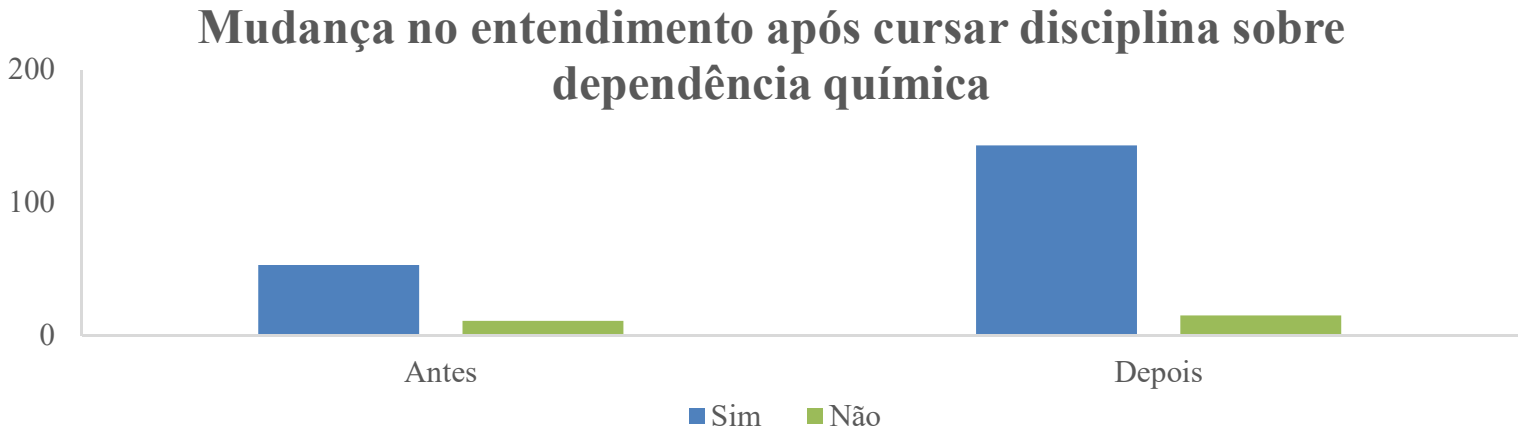

Figura 40: Frequência das respostas obtidas sobre a mudança no entendimento dos estudantes após cursarem disciplinas que abordam o tema uso de drogas e dependência química, apontadas na Questão 14. As colunas representam as frequências da alternativa Sim Antes = 13 e Depois $=52$ e Não Antes $=4$ e Depois $=4(\mathrm{n}$ total $=73)$.

\begin{tabular}{l} 
16. Você acha que a formação sobre uso de drogas e dependência \\
química é relevante no seu curso? \\
\hline Sim \\
\hline Não
\end{tabular}

$\mathrm{Na}$ Questão 16, o teste $t$ não-pareado de Student não revelou diferença significativa entre as respostas obtidas nas alternativas $\operatorname{Sim}$ Antes $(\bar{X}=35 ; \mathrm{EPM}=4.31)$ e Depois $(\bar{X}=$ 34.1; $\mathrm{EPM}=2.85), t=0.16$ e $\mathrm{p}=0.86$ e $N a ̃ o$ Antes $(\bar{X}=0.87 ; \mathrm{EPM}=0.35)$ e Depois $(\bar{X}=$ $1.12 ; \mathrm{EPM}=0.71), t=0.31 \mathrm{e} \mathrm{p}=0.75($ Figura 41$)$.

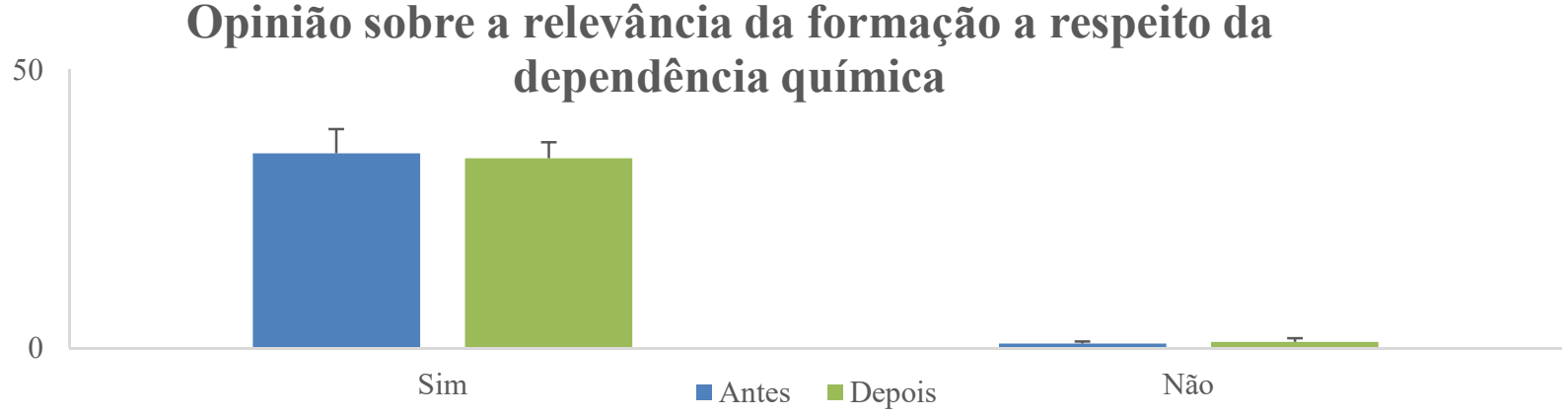

Figura 41: Opinião dos estudantes acerca da relevância da formação sobre dependência 
química em seu curso antes e depois de cursar a(s) disciplina(s) da área de Farmacologia. As colunas representam as médias e as barras o EPM. $\mathrm{p}<0,05$, de acordo com o teste $t$ nãopareado de Student. Antes $(\mathrm{n}=289)$ e Depois $(\mathrm{n}=281)$.

\begin{tabular}{l}
\hline $\begin{array}{l}\text { 17. Você já usou alguma(s) da(s) substância(s) a seguir? (Assinale } \\
\text { todas as alternativas que se aplicam) }\end{array}$ \\
\hline Álcool \\
\hline Alucinógeno (LSD, chá de ayahuasca) \\
\hline Anfetamina (metanfetamina, metilfenidato) \\
\hline Benzodiazepínico ou barbitúrico (clonazepam, fenobarbital) \\
\hline Cigarro de nicotina \\
\hline Cocaína ou merla ou crack \\
\hline Esteróide anabolizante (testosterona) \\
\hline Inalantes (loló, lança-perfume) \\
\hline Maconha ou haxixe ou skank \\
\hline Opiáceo (codeína, morfina, meperidina)/ Opióide (heroína) \\
\hline
\end{tabular}

Na Questão 17, o teste $t$ não-pareado de Student revelou que não houve diferença significativa em nenhuma das alternativas (Figura 42): Álcool Antes $(\bar{X}=31 ; \mathrm{EPM}=4.02) \mathrm{e}$ Depois $(\bar{X}=32.9 ; \mathrm{EPM}=2.72), t=-0.38$ e $\mathrm{p}=0.70$, Alucinógeno Antes $(\bar{X}=2.88 .63 ; \mathrm{EPM}$ $=0.71)$ e Depois $(\bar{X}=5.25 ; \mathrm{EPM}=0.97), t=-1.96$ e $\mathrm{p}=0.07$, Anfetamina Antes $(\bar{X}=1.38$; $\mathrm{EPM}=0.46)$ e Depois $(\bar{X}=2.63 ; \mathrm{EPM}=0.68), t=-1.52$ e $\mathrm{p}=0.15$, Benzodiazepínico ou barbitúrico Antes $(\bar{X}=1.63 ; \mathrm{EPM}=0.65)$ e Depois $(\bar{X}=3.50 ; \mathrm{EPM}=0.88), t=-1.70$ e $\mathrm{p}=$ 0.11 , Cigarro de nicotina Antes $(\bar{X}=9 ; \mathrm{EPM}=1.65)$ e Depois $(\bar{X}=10.88 ; \mathrm{EPM}=1.22), t=$ - 0.91 e p $=0.35$, Cocaína ou merla ou crack Antes $(\bar{X}=0.37 ; \mathrm{EPM}=0.26)$ e Depois $(\bar{X}=$ $1.73 ; \mathrm{EPM}=0.49), t=-1.78$ e $\mathrm{p}=0.09$, Esteroide anabolizante Antes $(\bar{X}=0.62 ; \mathrm{EPM}=$ $0.37)$ e Depois $(\bar{X}=0.50 ; \mathrm{EPM}=0.26), t=0.27$ e $\mathrm{p}=0.79$, Inalantes Antes $(\bar{X}=2.75 ; \mathrm{EPM}$ $=0.86)$ e Depois $(\bar{X}=4 ; \mathrm{EPM}=0.62), t=-1.17$ e $\mathrm{p}=0.26$, Cigarro de maconha Antes $(\bar{X}$ $=8.63 ; \mathrm{EPM}=1.88)$ e Depois $(\bar{X}=10.25 ; \mathrm{EPM}=1.44), t=-0.68$ e $\mathrm{p}=0.50$ e

Opiáceo/Opióide Antes $(\bar{X}=0.62 ; \mathrm{EPM}=0.26)$ e Depois $(\bar{X}=2.25 ; \mathrm{EPM}=0.77), t=-1.99$ $\mathrm{e} p=0.06$ 


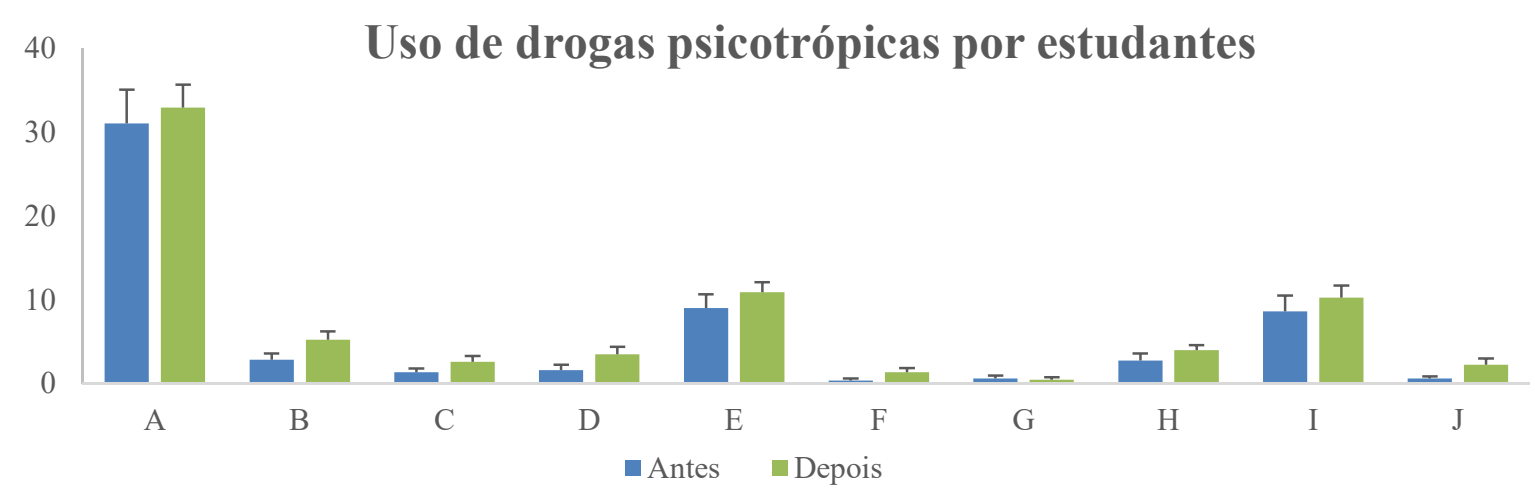

Figura 42: Uso de drogas psicotrópicas por estudantes antes e depois de cursar a(s) disciplina(s) da área de Farmacologia. As colunas representam as médias das alternativas A Álcool; B - Alucinógeno; C - Anfetamina; D - Benzodiazepínico ou barbitúrico; E - Cigarro de nicotina; F - Cocaína ou merla ou crack; G - Esteroide anabolizante; H - Inalantes; I Maconha ou haxixe ou skank e J - Opiáceo/Opióide e as barras o EPM. p < 0,05, de acordo com o teste $t$ não-pareado de Student. Antes $(\mathrm{n}=289)$ e Depois $(\mathrm{n}=281)$.

\begin{tabular}{l}
\hline 18 . Você usou alguma das substâncias da questão 17 nos últimos \\
30 dias? \\
\hline Sim \\
\hline Não \\
\hline Não se aplica
\end{tabular}

Na Questão 18, o teste $t$ não-pareado de Student não revelou diferença significativa em nenhuma das alternativas: $\operatorname{Sim}$ Antes $(\quad \bar{X}=15.6 ; \mathrm{EPM}=2.18)$ e Depois $(\bar{X}=18.5$; $\mathrm{EPM}=1.69), t=-1.04$ e $\mathrm{p}=0.31$, Não Antes $(\bar{X}=16.5 ; \mathrm{EPM}=2.09)$ e Depois $(\bar{X}=16.9$; $\mathrm{EPM}=1.63), t=-.14$ e $\mathrm{p}=0.88$ e Não se aplica Antes $(\bar{X}=4.25 ; \mathrm{EPM}=0.81)$ e Depois $(\bar{X}$ $=2.50 ; \mathrm{EPM}=0.42), t=1.90$ e $\mathrm{p}=0.07($ Figura 43).

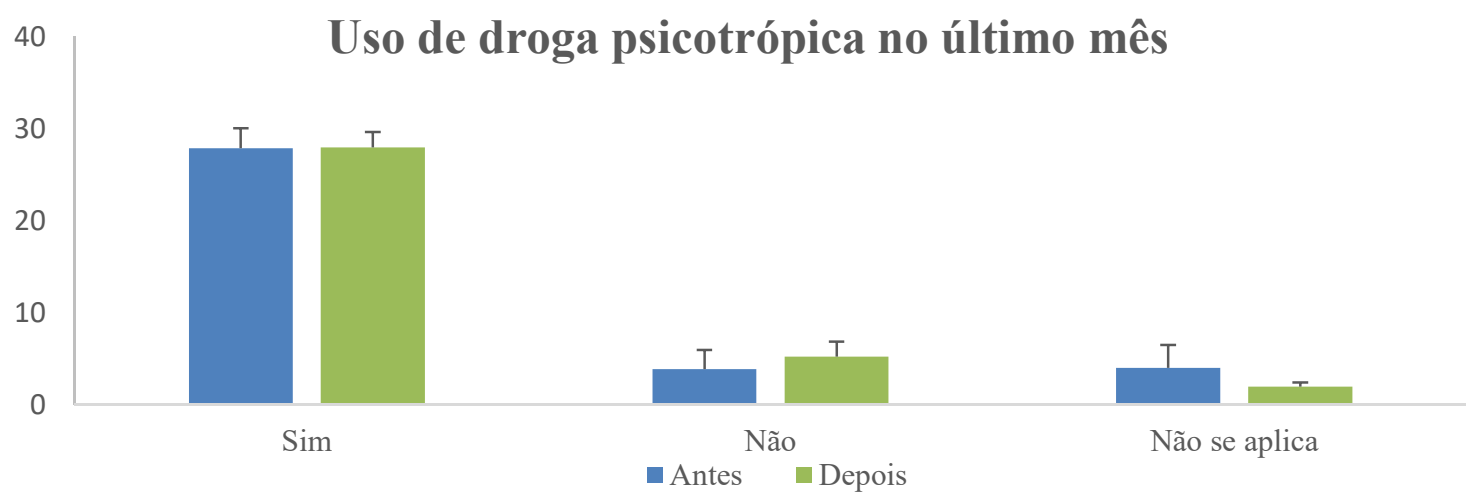

Figura 43: Uso de drogas psicotrópicas nos últimos 30 dias em comparação entre antes e depois de cursar a(s) disciplina(s) da área de Farmacologia. As colunas representam as médias 
e as barras o EPM. $\mathrm{p}<0,05$, de acordo com o teste $t$ não-pareado de Student. Antes $(\mathrm{n}=289)$ e Depois $(n=281)$.

\begin{tabular}{l}
$\begin{array}{l}\text { 21. Você já usou medicamento(s) psicotrópico(s) sem prescrição } \\
\text { médica? }\end{array}$ \\
\hline Sim \\
\hline Não \\
\hline
\end{tabular}

$\mathrm{Na}$ Questão 21, o teste $t$ não-pareado de Student revelou que não houve diferença significativa entre as respostas obtidas nas alternativas $\operatorname{Sim}$ Antes $(\quad \bar{X}=4.25 ; \mathrm{EPM}=1.16)$ e Depois $(\bar{X}=4.25 ; \mathrm{EPM}=0.41), t=0$ e $\mathrm{p}=1$ e Não Antes $(\bar{X}=31.6 ; \mathrm{EPM}=3.95)$ e Depois $(\bar{X}=31 ; \mathrm{EPM}=2.76), t=0.13$ e $\mathrm{p}=0.89$ (Figura 44).

\section{Uso de medicamento(s) psicotrópico(s) sem prescrição}

40

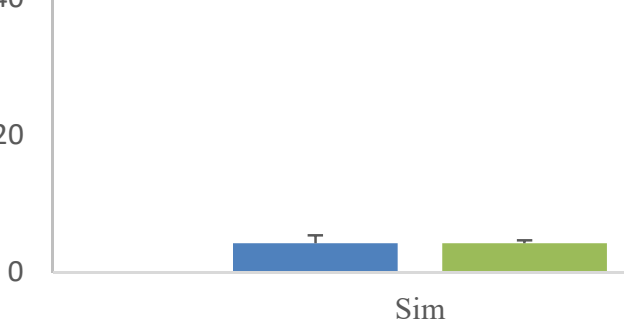

\section{médica}

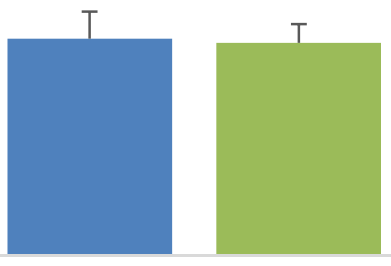

Não

- Antes Depois

Figura 44: Uso de medicamento(s) psicotrópico(s) sem prescrição médica pelos estudantes antes e depois de cursar a(s) disciplina(s) da área de Farmacologia. As colunas representam as médias e as barras o EPM. p $<0,05$, de acordo com o teste $t$ não-pareado de Student. Antes (n $=289)$ e Depois $(\mathrm{n}=281)$.

\begin{tabular}{l} 
22. Se você julgar que existe tratamento para dependência química, \\
como deveria ser? (Assinale todas as alternativas que se aplicam) \\
\hline Eletrochoque \\
\hline Internação em clínica de orientação religiosa \\
\hline Internação voluntária \\
\hline Internação compulsória \\
\hline Psicoterapia \\
\hline Uso de medicamentos \\
\hline Não há tratamento \\
\hline Nenhum dos tratamentos anteriores
\end{tabular}

Na Questão 22, o teste $t$ não-pareado de Student mostrou que não houve diferença $\bar{X}$ significativa entre nenhuma das respostas (Figura 23): Eletrochoque Antes $(\bar{X}=0.12 ; \mathrm{EPM}=$ 0.12) e Depois $(\bar{X}=0.50 ; \mathrm{EPM}=0.26), t=-1.27$ e $\mathrm{p}=0.22$, Internação em clínica de orientação religiosa Antes $(\bar{X}=5.75 ; \mathrm{EPM}=1.03)$ e Depois $(\bar{X}=6 ; \mathrm{EPM}=1.07), t=-0.16$ e $\mathrm{p}=0.86$, Internação voluntária Antes $(\bar{X}=28.1 ; \mathrm{EPM}=3.81)$ e Depois $(\bar{X}=28.9 ; \mathrm{EPM}=$ 
$2.98), t=-0.15$ e $\mathrm{p}=0.87$, Internação compulsória Antes $(\bar{X}=10 ; \mathrm{EPM}=1.40)$ e Depois ( $\bar{X}=9.13 ; \mathrm{EPM}=1.39), t=0.44$ e $\mathrm{p}=0.66$, Psicoterapia Antes $(\bar{X}=29.8 ; \mathrm{EPM}=4.42) \mathrm{e}$ Depois $(\bar{X}=28.8 ; \mathrm{EPM}=3.10), t=-0.18$ e $\mathrm{p}=0.85$, Uso de medicamentos Antes $(\bar{X}=$ $15.4 ; \mathrm{EPM}=2.68)$ e Depois $(\bar{X}=22.8 ; \mathrm{EPM}=2.85), t=1.88$ e $\mathrm{p}=0.08$, Não há tratamento Antes $(\bar{X}=0 ; \mathrm{EPM}=0)$ e Depois $(\bar{X}=0 ; \mathrm{EPM}=0), t=0$ e $\mathrm{p}=1$ e Nenhuma dos tratamentos anteriores Antes $(\bar{X}=0.37 ; \mathrm{EPM}=0.26)$ e Depois $(\bar{X}=0.37 ; \mathrm{EPM}=0.26), t=$ 0 e $p=1$ (Figura 45).

\section{Conhecimento sobre tratamentos para dependência}

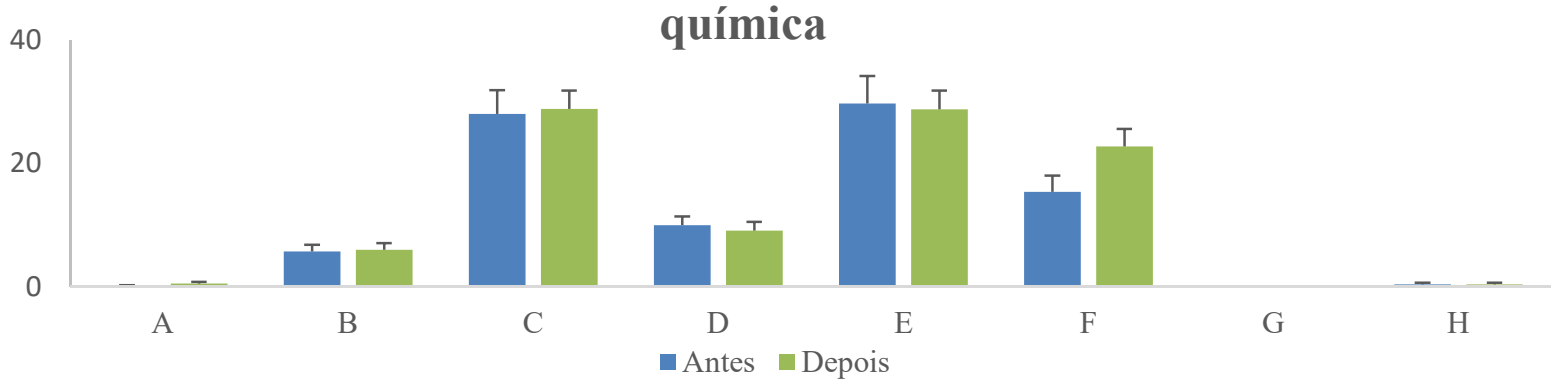

Figura 45: Conhecimento acerca das modalidades de tratamento para a dependência química antes e depois de cursar a(s) disciplina(s) da área de Farmacologia. As colunas representam as médias das alternativas A - Eletrochoque, B - Internação em clínica de orientação religiosa, C - Internação voluntária, D - Internação Compulsória, E- Psicoterapia, F - Uso de medicamentos, G - Não há tratamento, H - Nenhum dos anteriores e as barras o EPM. $\mathrm{p}<$ 0,05 , de acordo com o teste $t$ não-pareado de Student. Antes $(\mathrm{n}=289)$ e Depois $(\mathrm{n}=281)$.

\begin{tabular}{l}
$\begin{array}{l}\text { 23. Você conhece uma modalidade de tratamento chamada } \\
\text { redução de danos? }\end{array}$ \\
\hline Sim \\
\hline Não
\end{tabular}

Na Questão 23, o teste $t$ não-pareado de Student revelou que não houve diferença significativa nas respostas das alternativas $\operatorname{Sim}$ Antes $(\bar{X}=25 ; \mathrm{EPM}=3.94)$ e Depois $(\bar{X}=$ 26.4; $\mathrm{EPM}=2.28), t=-0.30$ e $\mathrm{p}=0.76$ e Não Antes $(\bar{X}=10.7 ; \mathrm{EPM}=1.06)$ e Depois $(\bar{X}=$ $3.01 ; \mathrm{EPM}=1.33), t=1.10$ e $\mathrm{p}=0.28($ Figura 46$)$ 


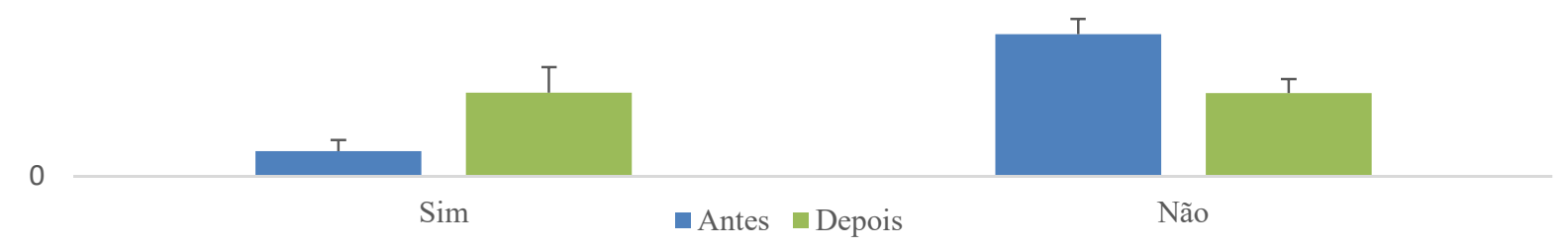

Figura 46: Conhecimento sobre redução de danos antes e depois de cursar a(s) disciplina(s) da área de Farmacologia. As colunas representam as médias e as barras o EPM. p < 0,05, de acordo com o teste $t$ não-pareado de Student. Antes $(\mathrm{n}=289)$ e Depois $(\mathrm{n}=281)$.

Na última pergunta do questionário, Questão 25, os estudantes definiram o dependente químico usando apenas uma palavra.

\section{Avaliação da comparação entre os cursos do grupo experimental e do grupo controle:}

Os resultados das comparações intergrupos, entre os 944 questionários oriundos dos cursos Ciências Farmacêuticas (diurno e noturno), Enfermagem, Farmácia, Medicina, Nutrição, Odontologia e Psicologia (grupo experimental), Gestão em Saúde Coletiva, Fisioterapia, Fonoaudiologia, Saúde Coletiva e Terapia Ocupacional (grupo controle), foram revelados pela Análise de Variância de uma via (ANOVA One-Way). O teste post-hoc usado para identificar as diferenças foi o teste de Student-Newman-Keuls $(\mathrm{p}<0,05)$.

Os dados relativos a cada questão da comparação intercursos que não apresentaram diferença significativa são apresentados em seguida. Na Questão 1, acerca da idade dos participantes analisados nesse teste, a média geral de idade foi 24.7 anos (Figuras 47 e 48).

\section{Média geral da frequência da idade dos estudantes}

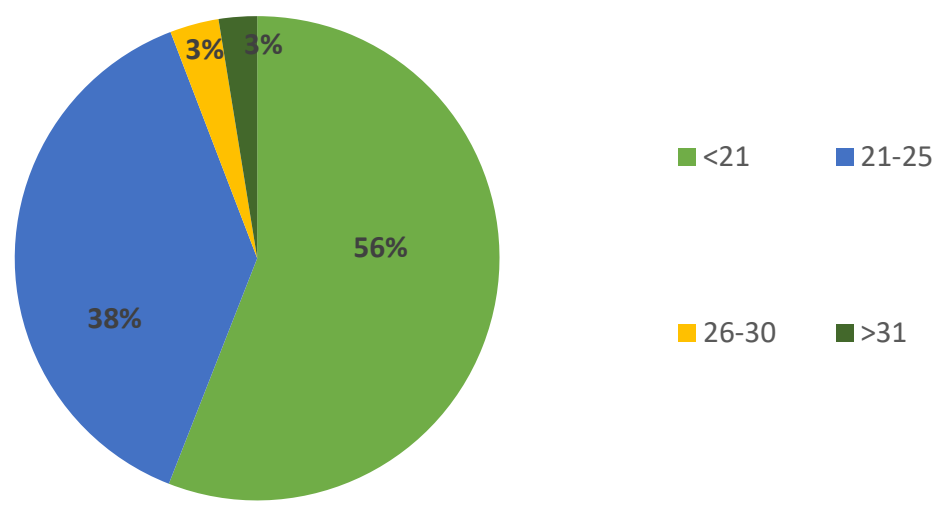

Figura 47: Distribuição da média geral da frequência (em \%) da idade dos estudantes dos cursos da área de saúde da Universidade de Brasília $(\mathrm{n}=944)$. 


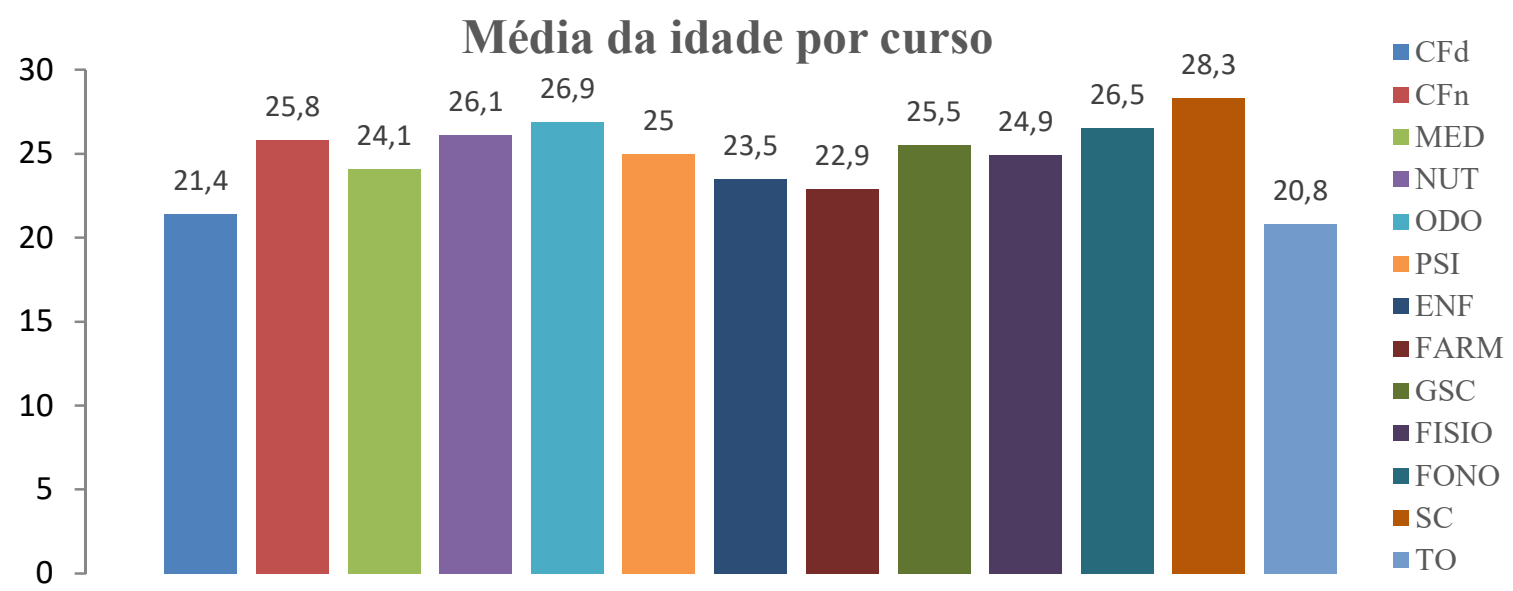

Figura 48: Distribuição da média geral da idade dos estudantes da área de saúde da Universidade de Brasília por curso. Ciências Farmacêuticas diurno (CFd); Ciências Farmacêuticas noturno (CFn); Medicina (Med); Nutrição (Nut); Odontologia (Odo); Psicologia (Psi); Enfermagem (Enf); Farmácia (Farm); Gestão em Saúde Coletiva (GSC); Fisioterapia (Fisio); Fonoaudiologia (Fono); Saúde Coletiva (SC) e Terapia Ocupacional (TO) ( $\mathrm{n}$ total $=944$ ). As colunas representam os cursos e as barras as frequências.

Quanto ao sexo dos estudantes, os resultados da Questão 2 mostram que 725 são do sexo feminino e 219 do masculino (Figuras 49 e 50).

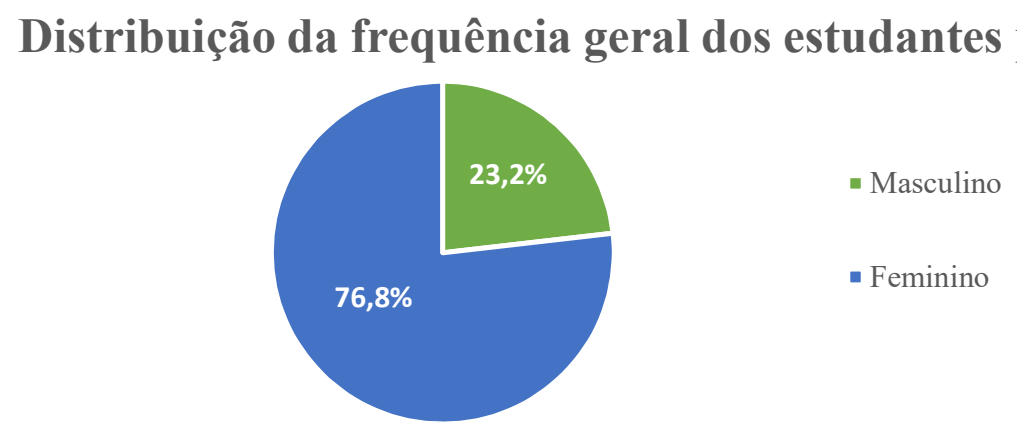

Figura 49: Distribuição da frequência geral dos estudantes (em \%) por sexo nos cursos da área de saúde. Feminino = 725 e Masculino $=219(\mathrm{n}$ total $=944)$.

\section{Distribuição dos estudantes por sexo e por curso}

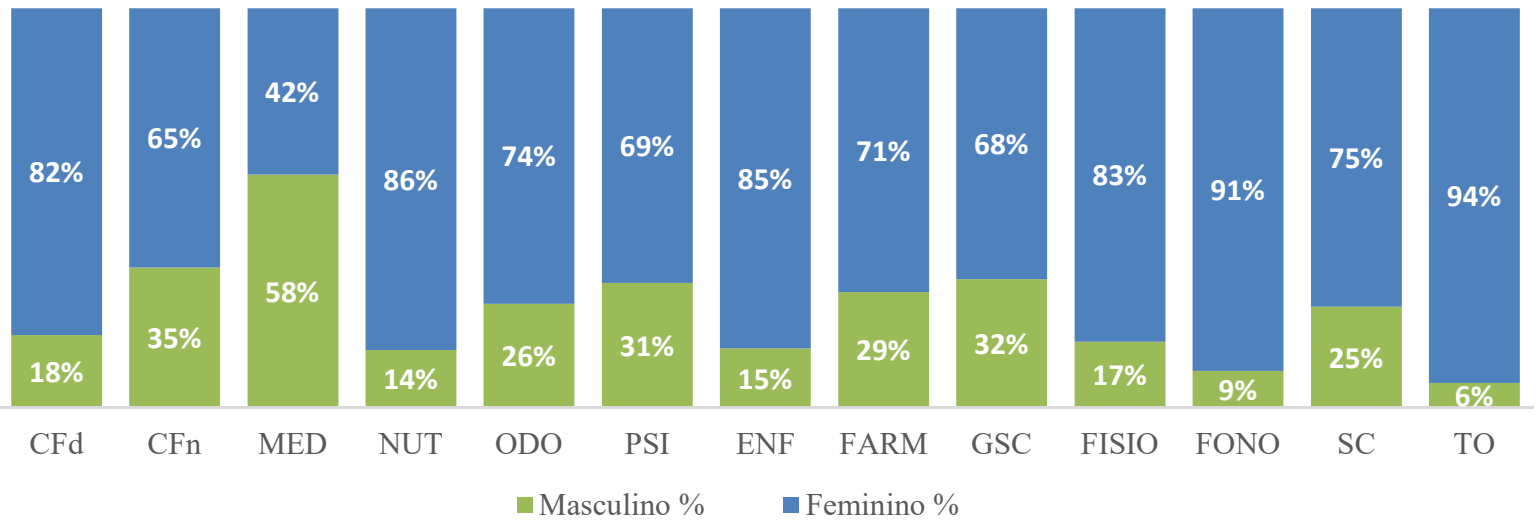

Figura 50: Distribuição da frequência dos estudantes (em \%) por sexo e por curso. Ciências Farmacêuticas diurno $(\mathrm{CFd})$ : Feminino $(\mathrm{F})=63$ e Masculino $(\mathrm{M})=14$; Ciências 
Farmacêuticas noturno (CFn): $\mathrm{F}=37$ e $\mathrm{M}=20$; Medicina (Med): $\mathrm{F}=21$ e $\mathrm{M}=28$; Nutrição (Nut): $\mathrm{F}=59$ e $\mathrm{M}=10$; Odontologia (Odo): $\mathrm{F}=34$ e $\mathrm{M}=12$; Psicologia (Psi): $\mathrm{F}=66$ e $\mathrm{M}=$ 30; Enfermagem (Enf): $\mathrm{F}=80$ e $\mathrm{M}=14$; Farmácia (Farm): $\mathrm{F}=59$ e $\mathrm{M}=24$; Gestão em Saúde Coletiva (GSC): $F=40$ e $M=19$; Fisioterapia (Fisio): $F=86$ e $M=17$;

Fonoaudiologia (Fono): $\mathrm{F}=59$ e $\mathrm{M}=6$; Saúde Coletiva (SC): $\mathrm{F}=62$ e $\mathrm{M}=21$ e Terapia Ocupacional (TO): $\mathrm{F}=60$ e $\mathrm{M}=4$ ( $\mathrm{n}$ total $=944)$.

A Questão 3, acerca do curso, garantia a proveniência dos estudantes, ao passo que a Questão 4, a respeito do estudante ter cursado ou não Farmacologia, confirmava seu posicionamento nos grupos experimental ou controle.

O estudante que afirmasse ter cursado alguma disciplina que abordasse a temática do uso de drogas e da dependência química na Questão 13, deveria especificar qual(is) foi(ram) essa(s) disciplina(s) na Questão 14. As frequências das disciplinas do grupo experimental (Ciências Farmacêuticas diurno, Ciências Farmacêuticas noturno, Medicina, Nutrição, Odontologia, Enfermagem e Farmácia) foram apresentadas na Figura 39. Em seguida, são apresentadas as frequências das disciplinas dos cursos do grupo controle (Figura 51).
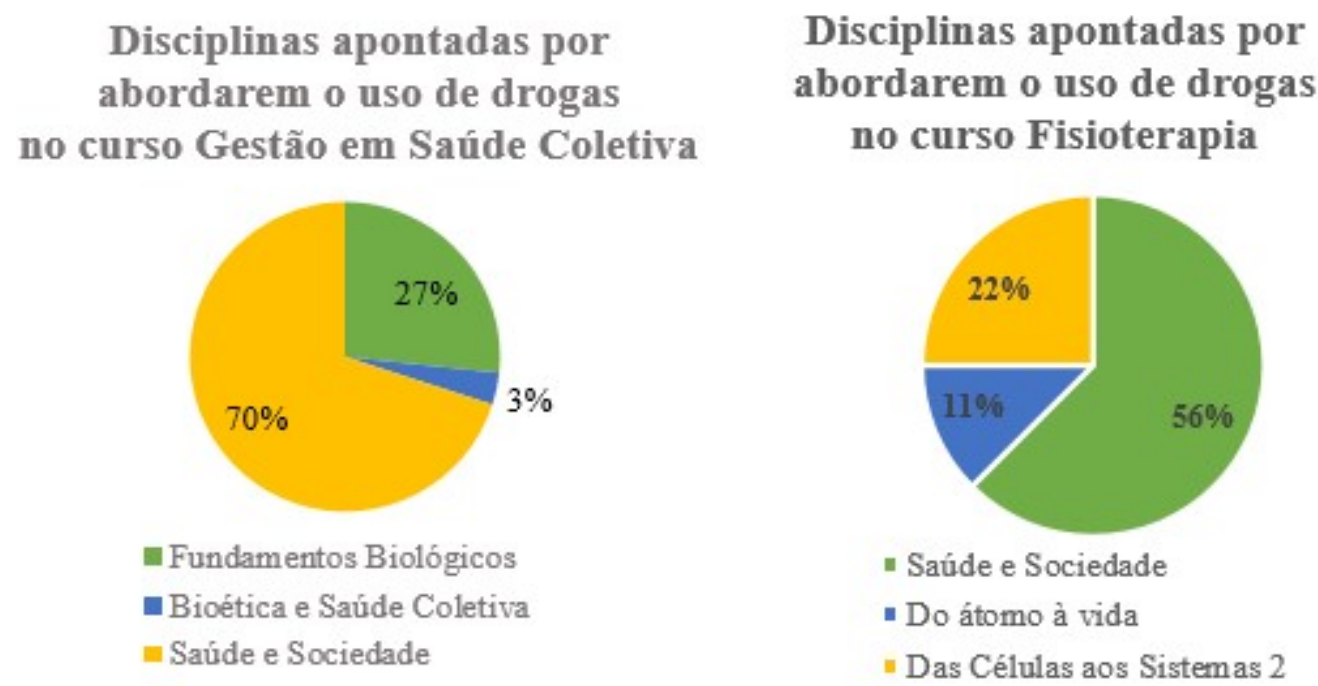


\section{Disciplinas apontadadas por abordadem o uso de drogas no curso Fonoaudiologia}

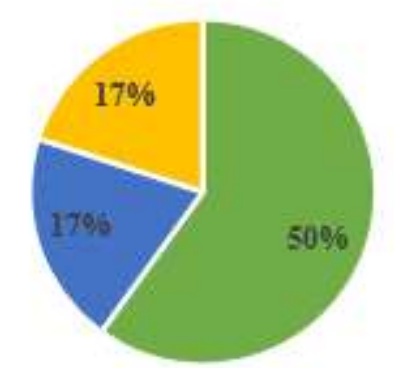

- Saúde e Sociedade

- Das Células aos Sistemas 2

* Epidemiologia

\section{Disciplinas que apontadas por abordarem o uso de drogas no curso Saúde Coletiva}

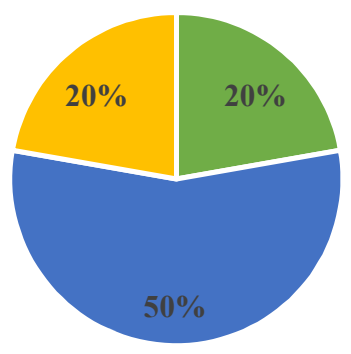

- Pensamento Social em Saúde Coletiva

- Saúde e Sociedade

- Epidemiologia

Disciplinas apontadas por abordarem

o uso de drogas

no curso Terapia Ocupacional

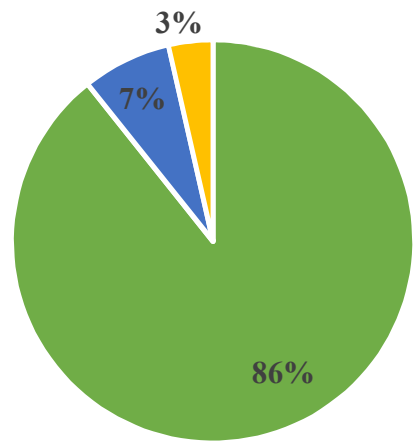

- Terapia Ocupacional na Reabilitação: Intervenção

- Fundamentos de Terapia Ocupacional

- Recursos Terapêuticos

Figura 51: Frequência das disciplinas que abordam o uso de drogas e a dependência química mencionadas na Questão 14 pelos cursos do grupo controle: Gestão em Saúde Coletiva n = 22 , Fisioterapia $n=7$, Fonoaudiologia $n=5$, Saúde Coletiva $n=10$ e Terapia Ocupacional $=$ $28(\mathrm{n}$ total $=72)$.

A Questão 15 perguntava se houve mudança no entendimento dos estudantes a respeito do uso de drogas e da dependência química após cursar a(s) disciplina(s) listada(s) na Questão 14. Abaixo é listada a frequência das alternativas Sim e Não obtidas nos cursos do grupo controle: Gestão em Saúde Coletiva, Fisioterapia, Fonoaudiologia, Saúde Coletiva e Terapia Ocupacional (Figura 52). 


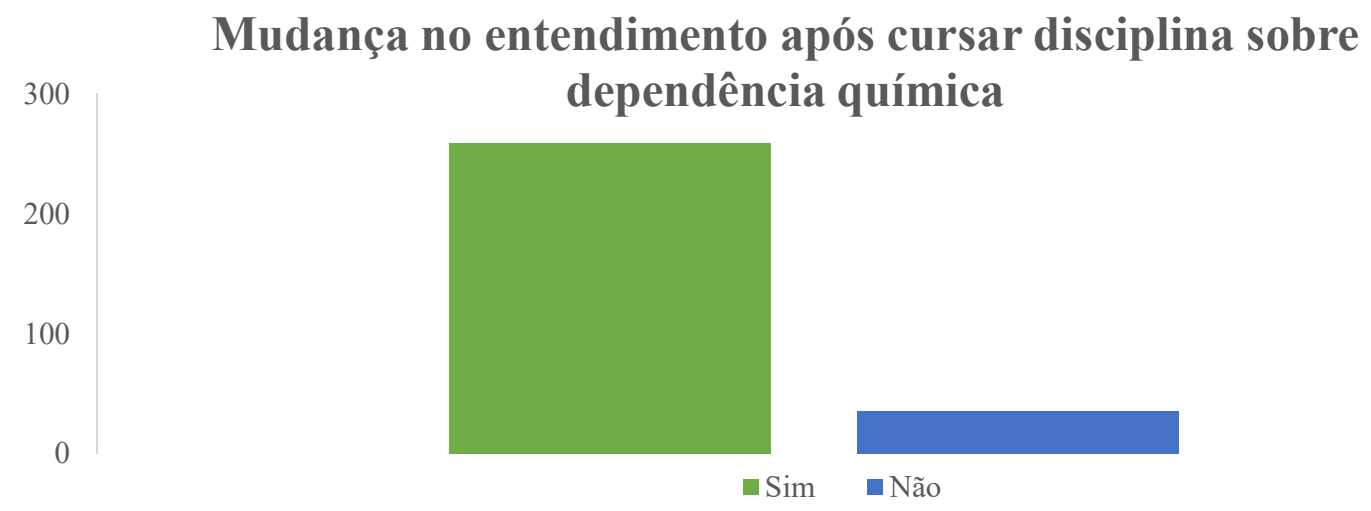

Figura 52: Frequência das respostas obtidas acerca da mudança no entendimento dos estudantes após cursarem disciplinas que abordam o uso de drogas e a dependência química apontadas na Questão 14. As colunas representam as frequências das alternativas $\operatorname{Sim}=259$ e Não $=35(\mathrm{n}$ total $=294)$.

\begin{tabular}{l}
\hline $\begin{array}{l}\text { 23. Você conhece uma modalidade de tratamento chamada } \\
\text { redução de danos? }\end{array}$ \\
\hline Sim \\
\hline Não
\end{tabular}

Na Questão 23, a ANOVA One-Way, seguida pelo teste de Student-Newman- Keuls, revelou que não houve diferença significativa na alternativa $\operatorname{Sim} \mathrm{F}_{(12,931)}=1.03$ e p $=0.47$ (Figura 53).

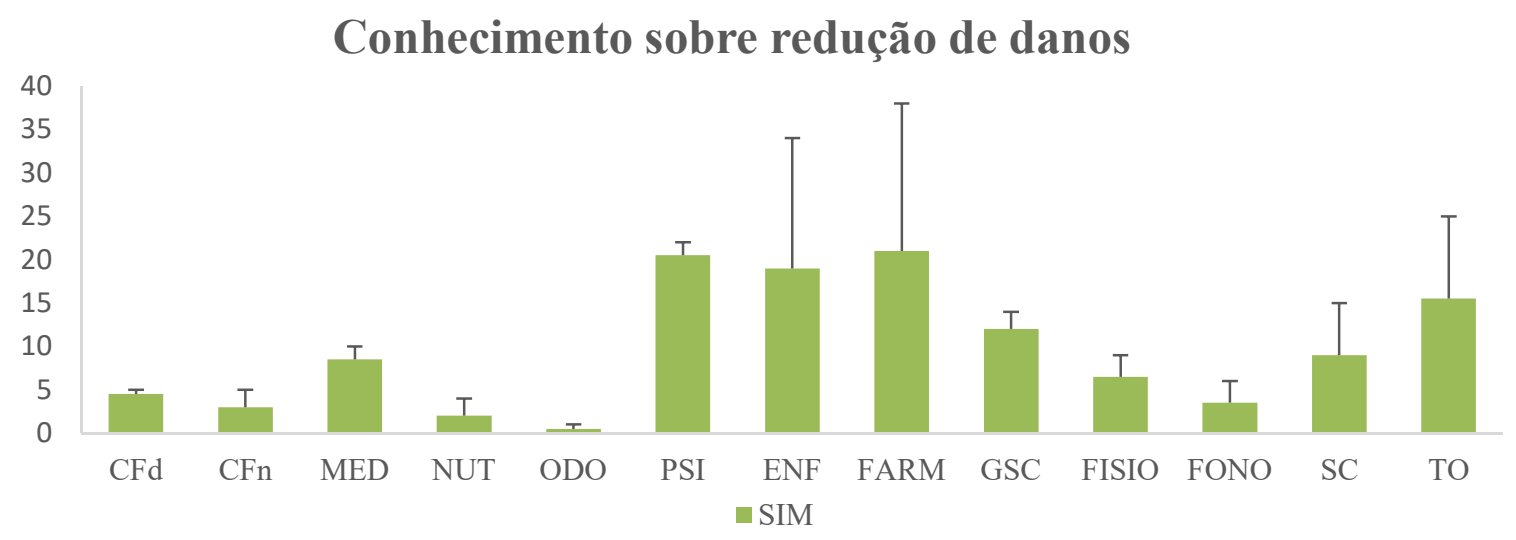

Figura 53: Conhecimento sobre redução de danos. A colunas representam as médias e as barras o EPM. $\mathrm{p}<0,05$, de acordo com a ANOVA One-Way $(\mathrm{n}=944)$.

\begin{tabular}{l}
\hline $\begin{array}{l}\text { 24. Qual a principal característica da redução de danos? } \\
\text { (Assinale todas as alternativas que se aplicam) }\end{array}$ \\
\hline Não tem eficácia \\
\hline Pode envolver tratamento medicamentoso \\
\hline Pode envolver uso de droga que cause dependência \\
\hline Tem como objetivo a abstinência \\
\hline Não sei \\
\hline
\end{tabular}


Na Questão 24, a ANOVA One-Way, seguida pelo teste de Student-Newman- Keuls, revelou que não houve diferença significativa em nenhuma das alternativas: Não tem eficácia $\mathrm{F}_{(12,931)}=0.75$ e $\mathrm{p}=0.68 ;$ Envolve uso de medicamento $\mathrm{F}_{(12,931)}=1.27$ e $\mathrm{p}=0.33 ;$ Pode envolver uso de droga que cause dependência $\mathrm{F}_{(12,931)}=0.96$ e $\mathrm{p}=0.55 ;$ Tem como objetivo a abstinência $\mathrm{F}_{(12,931)}=1.53$ e $\mathrm{p}=0.22$ e Não sei $\mathrm{F}_{(12,931)}=0.71$ e $\mathrm{p}=0.72$ (Figura 54).

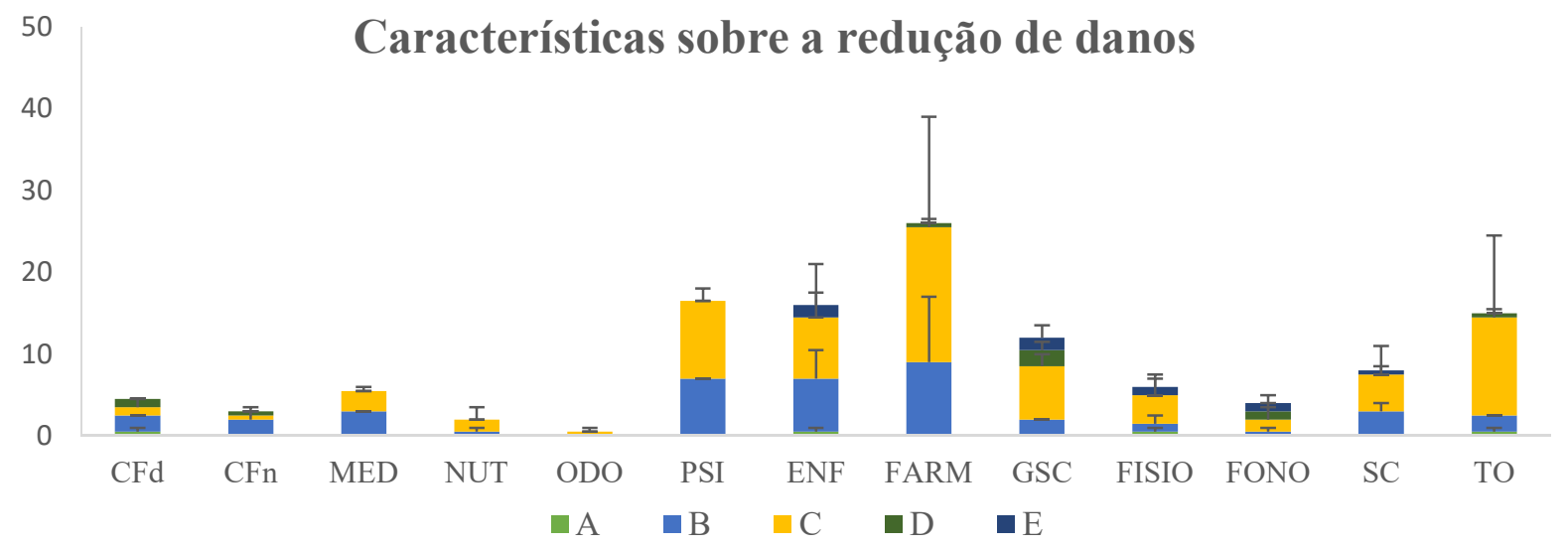

Figura 54: Características sobre a redução de danos. As colunas representam as médias das alternativas: A - Não tem eficácia, B - Envolve tratamento medicamentoso, C - Pode envolver uso de droga que cause dependência, D - Tem como objetivo a abstinência, E - Não sei e as barras o EPM. $\mathrm{p}<0,05$, de acordo com a ANOVA One-Way $(\mathrm{n}=944)$.

Na Questão 25, os estudantes usaram uma palavra para definir o dependente químico e as respostas das frequências mais altas e que apareceram em todos os cursos, doente e necessitado, foram analisadas por meio da ANOVA One-Way, seguida pelo teste de StudentNewman-Keuls (Figura 55). Não houve diferença significativa para a palavra "Doente" $\left(F_{(12,121)}=1.89\right.$ e $\left.p=0.13\right)$, nem para a palavra "Necessitado" $\left(F_{(12,121)}=0.77\right.$ e $\left.p=0.66\right)$.

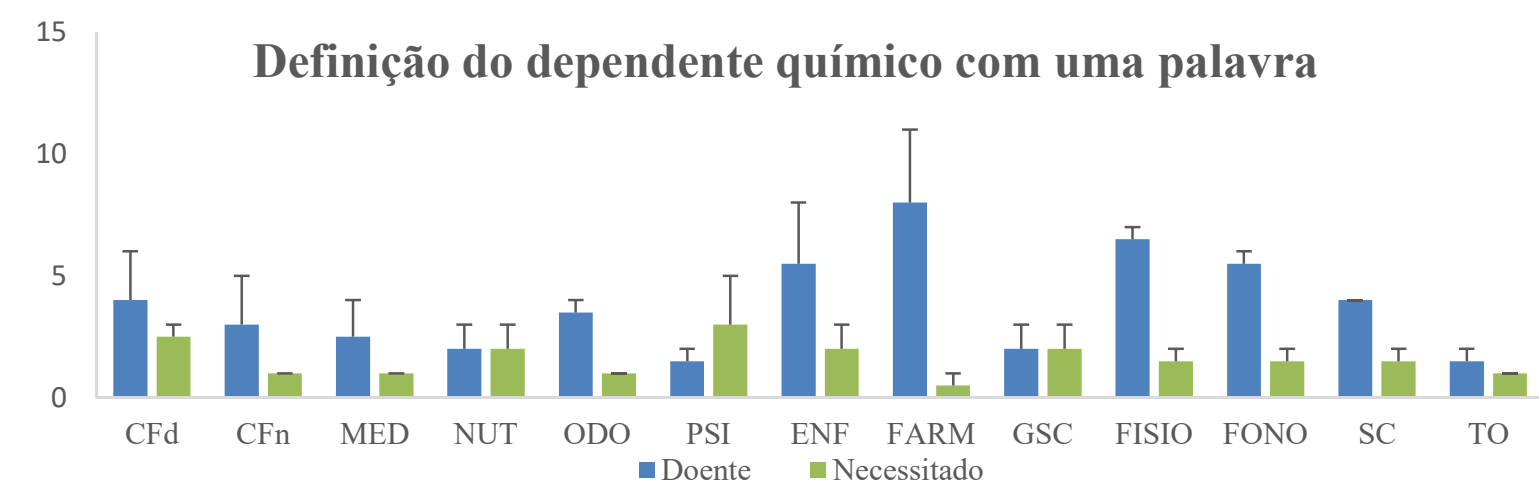

Figura 55: As colunas representam as médias das palavras Doente $(\mathrm{n}=100)$ e Necessitado $(\mathrm{n}$ =34) e as barras o EPM. $\mathrm{p}<0,05$, de acordo com a ANOVA One-Way ( $\mathrm{n}$ total = 134). 
As combinações com maior frequência de resposta, obtidas a partir das questões em que havia a possibilidade de assinalar mais de uma alternativa, analisadas a partir da ANOVA One-Way e seguidas pelo teste de Student-Newman-Keuls $(\mathrm{p}<0,05)$, são mostradas a seguir.

Na Questão 12, a análise da combinação, por meio da ANOVA One-Way, seguida pelo teste de Student-Newman-Keuls, entre as alternativas Aulas expositivas, Congressos, Cursos de extensão, Palestras e Projeto de iniciação científica, revelou que não houve diferença significativa $\mathrm{F}_{(1,943)}=1.45 \mathrm{e} \mathrm{p}=0.25$ (Figura 56).

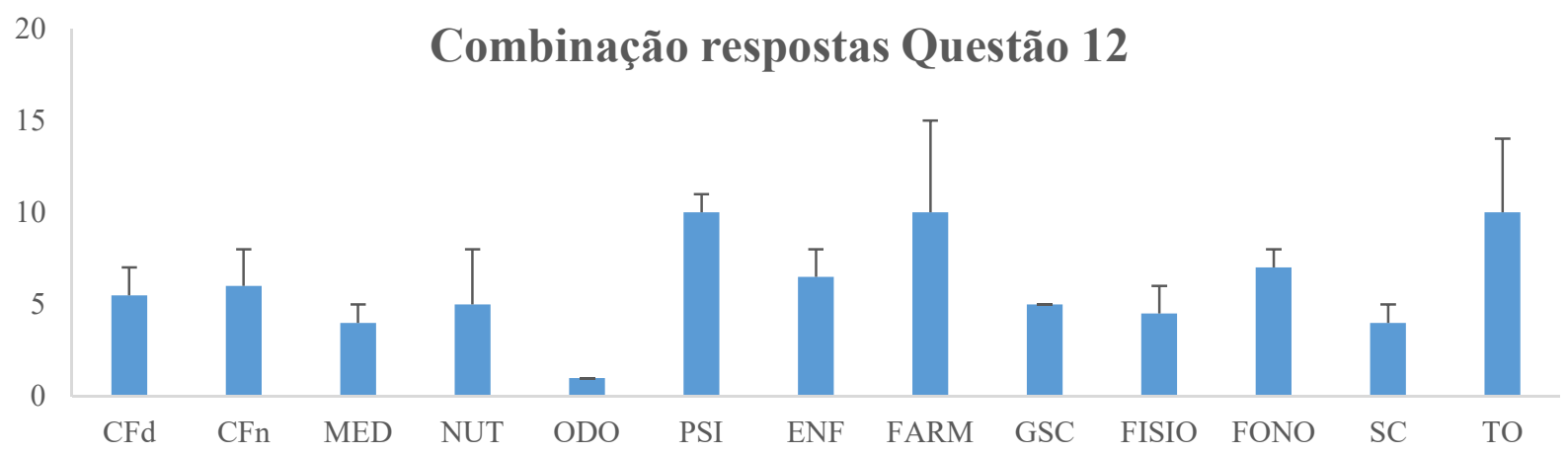

Figura 56: As colunas representam as médias e as barras o EPM da combinação das respostas Aulas expositivas, Congressos, Cursos de extensão, Palestras e Projeto de iniciação científica da Questão 12. $\mathrm{p}<0,05$ de acordo com ANOVA One-Way $(\mathrm{n}=158)$.

Na Questão 17, a análise da combinação, por meio da ANOVA One-Way, seguida pelo teste de Student-Newman-Keuls, entre as alternativas com maior frequência nessa questão (Álcool, Cigarro de nicotina e Maconha ou haxixe ou skank) revelou que não houve diferença significativa $\mathrm{F}_{(1,943)}=2.33$ e $\mathrm{p}=0.07$ (Figura 57).

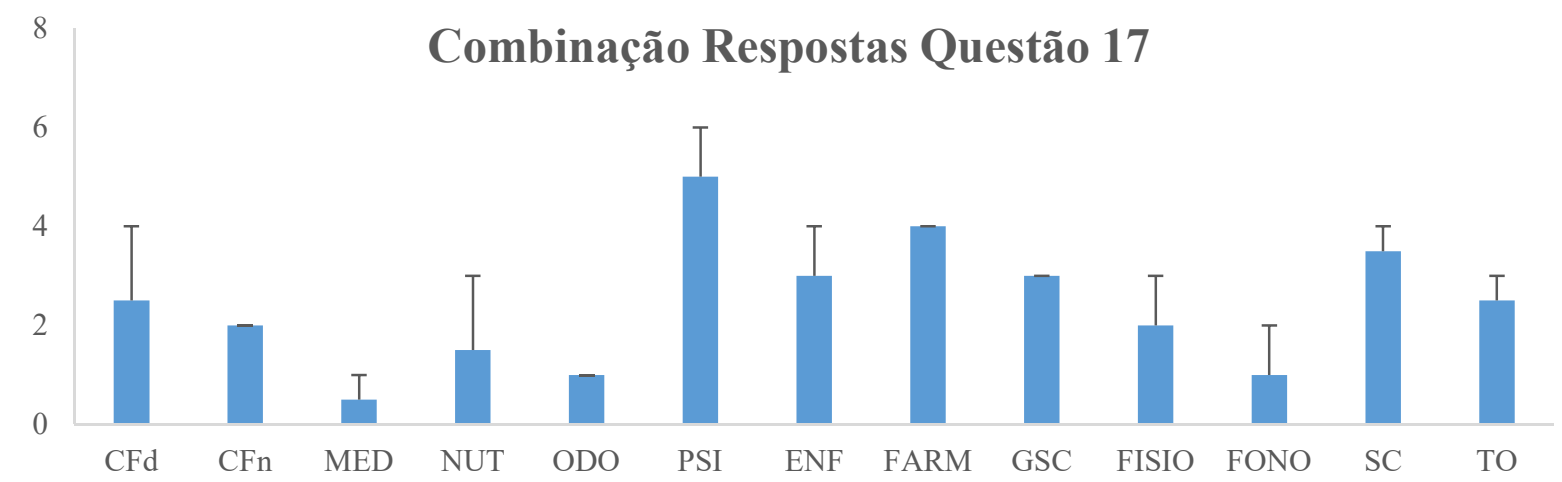

Figura 57: As colunas representam as médias e as barras o EPM da combinação das respostas Álcool, Cigarro de nicotina e Maconha ou haxixe ou skank da Questão 17. p <0,05 de acordo com ANOVA One-Way (n total =64). 
Na Questão 22, a análise da combinação, por meio da ANOVA One-Way, seguida pelo teste de Student-Newman-Keuls, entre as alternativas Internação voluntária e Psicoterapia revelou que não houve diferença significativa $\mathrm{F}_{(1,943)}=1.41$ e $\mathrm{p}=0.27$ (Figura $58)$.

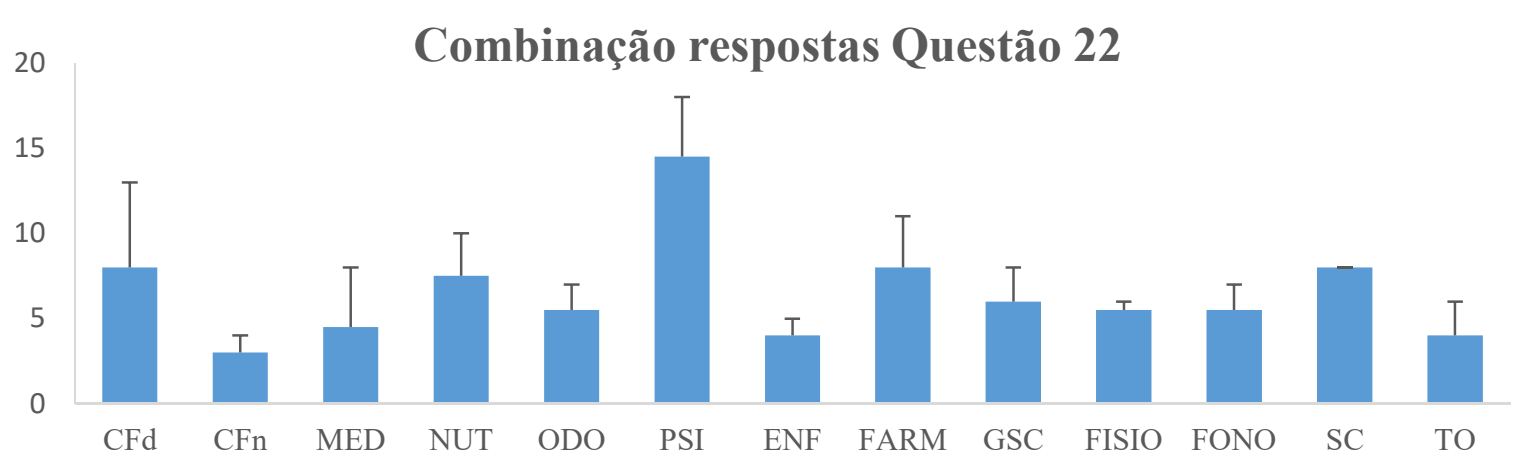

Figura 58: Combinação mais frequente entre as alternativas Internação voluntária e Psicoterapia da Questão 22. As colunas representam as médias e as barras o EPM. p < 0,05, de acordo com a ANOVA One-Way (n total $=153$ ). 